Journal of Patient-Centered coAdvocateAuroraHealth

Research and Reviews

Volume 5

Issue 1 - Health Disparities and Inequities: Part

II

$4-2-2018$

\title{
Abstracts From the 24th Annual Health Care Systems Research Network Conference, April 11-13, 2018, Minneapolis, Minnesota
}

Follow this and additional works at: https://aah.org/jpcrr

Part of the Diseases Commons, Health and Medical Administration Commons, Health Information Technology Commons, Medical Specialties Commons, Mental and Social Health Commons, Other Medicine and Health Sciences Commons, and the Public Health Commons

\section{Recommended Citation}

Abstracts from the 24th annual Health Care Systems Research Network Conference, April 11-13, 2018, Minneapolis, Minnesota. J Patient Cent Res Rev. 2018;5:56-131. doi: 10.17294/2330-0698.1630

Published quarterly by Midwest-based health system Advocate Aurora Health and indexed in PubMed Central, the Journal of Patient-Centered Research and Reviews (JPCRR) is an open access, peer-reviewed medical journal focused on disseminating scholarly works devoted to improving patient-centered care practices, health outcomes, and the patient experience. 


\title{
Abstracts From the $24^{\text {th }}$ Annual Health Care Systems Research Network Conference, April 11-13, 2018, Minneapolis, Minnesota
}

\author{
Improving Health and Well-Being: Connecting Research and Practice
}

Founded in 1994, the Health Care Systems Research Network (HCSRN, formerly named the HMO Research Network) is a consortium of 18 research centers that are housed in communitybased health systems. Members of the HCSRN share a commitment to public domain research designed to improve health and health care delivery for individuals and populations.

The HCSRN's annual conference serves as a venue for research teams to disseminate scientific findings, stimulate new collaborations, and share insights about conducting research in real-world care-delivery settings. Abstracts accepted for presentation at HCSRN 2018 are published in this supplement of Journal of Patient-Centered Research and Reviews, the official journal of the conference. J Patient Cent Res Rev. 2018;5:56-131.

\section{CARE SYSTEM, COMMUNITY ENGAGEMENT}

\section{OA1.01 \\ Lessons Learned From a Program to Engage Frontline Staff in Improvement and Innovation}

Paula Lozano, ${ }^{1}$ Lorella Palazzo, ${ }^{1}$ Caitlin Morrison, ${ }^{1}$ Allen Cheadle ${ }^{1}$

${ }^{1}$ Kaiser Permanente Washington Health Research Institute, Seattle, WA

Background: Engaging frontline staff in problem-solving is a core feature of a learning health system. Clinicians and managers are close to patient care processes and well positioned to identify opportunities for improvement and devise potential solutions for piloting. These pilots may be innovative interventions or adaptations of evidence-based practices. Such efforts often face implementation challenges, and most local pilots are not successfully scaled or spread. Few models exist for systematically 1) engaging health care staff in creative problem-solving, 2) ensuring successful implementation of pilot interventions, 3) evaluating the pilots, and 4) using findings to inform decisions about scale and spread.

Methods: The Partnership for Innovation (PFI) program at Kaiser Permanente Washington (formerly Group Health Cooperative) was designed to support staff efforts to improve and innovate by funding promising pilots. This study examines the PFI experience, assesses program outcomes, and extracts learnings to inform the creation and sustainment of pilot incubators within integrated health systems.

Results: Health system staff submitted proposals that were reviewed by leaders. The PFI gave rise to and funded 50 intervention pilots between 2008 and 2016. Average grant size was $\$ 94,500$ (SD: $\$ 93,300$ ). All pilots had an evaluation component. Assessment of the first 7 years' experience with PFI ( $\mathrm{n}=38$ projects) identified problems and led to program redesign in 2015. In an effort to better align pilots with the health system's strategic goals, the redesign included designating executive sponsorship and promoting accountability for results. Examining the projects and subsequent implementation success using the Consolidated Framework for Implementation Research elucidates constructs in four domains that are particularly relevant for others considering similar efforts. Those constructs are 1) intervention complexity and strength of the evidence, 2) knowledge and beliefs of the individuals involved, 3) funding, partnership, alignment and sponsorship within the organizational setting, and 4) the opportunity to evaluate and reflect as part of an established process. Evaluation and reflection both supported learning in real time (formative evaluation) and informed decisions about spreading effective interventions and discontinuing ineffective or infeasible ones.

Conclusion: Health systems that wish to leverage frontline creativity in solving care delivery problems should consider these factors.

Keywords: program evaluation, research, engagement of stakeholders, health care organization, dissemination, implementation, innovation, quality improvement 
OA1.02

Challenges, Lessons Learned, and Progress in Expanding Health Plan Engagement With PCORnet

Kevin Fahey ${ }^{1}$

Iunaffiliated, Washington, DC

Background: In 2013, the Patient-Centered Outcomes Research Institute (PCORI) established PCORnet (National Patient-Centered Clinical Research Network), 11 clinical data research networks (CDRNs) and 18 patientpowered research networks, to create a national resource for conducting patient-centered clinical comparative effectiveness research. PCORnet has been challenged in engaging health plans with the CDRNs as collaborators sharing data and conducting research. With a common data model, PCORnet resembles other networks, including U.S. Food and Drug Administration's Sentinel system and Health Care Systems Research Network's virtual data warehouse. Lessons learned, particularly from Sentinel, have guided PCORnet's development. PCORnet is part of a national evidence-generation network that includes government and private entities such as manufacturers, health systems, and insurers generating evidence to support a learning health care system. Health plans are important partners for PCORnet - as sources of research questions and essential data for answering them as well as end users of research findings. Except for a few CDRN-specific links with individual plans, PCORnet began with few established links to health plans. In 2016, PCORI funded two large national health plans to join PCORnet as partners with the CDRNs.

Methods: This highlights key lessons from a PCORIfunded Engagement Award project focused on building collaboration by (PCORnet) and health plans. Updates on progress since the completion of the award project will be included.

Results: Among challenges for health plans engaging with PCORnet: establishing trust and governance for collaboration and use of data; efficiently merging claims and clinical data; determining the minimum data necessary for any purpose; and defining the business case for collaboration by each stakeholder.

Conclusion: Among the lessons learned: organizations will clearly be more motivated to share data when it is worth their while to participate; health plans may be interested in using PCORnet and sharing data to address a wide range of research questions in which they have a stake; public interest is a key motivating factor for sharing data through PCORnet; completeness of data is an objective that supports collaboration; and establishing bidirectional relationships between the plans and CDRNs is necessary to sustain collaboration.

Keywords: ownership, governance, health care organization, virtual data warehouse, evaluation research, engagement of stakeholders, research administration, contracting, operations, HCSRN structure
OA1.03

Real-Time Text Message Alerts to Emergency Physicians Identifying Potential Study Candidates Increases Clinical Trial Enrollment

Laura Simon, ${ }^{1}$ Adina Rauchwerger, ${ }^{1}$ Uli Chettipally, ${ }^{1}$ David Vinson, ${ }^{1}$ Margaret Warton, ${ }^{1}$ Anupam Kharbanda, ${ }^{2}$ Elyse O. Kharbanda, ${ }^{3}$ Dustin Ballard ${ }^{1}$

${ }^{1}$ Kaiser Permanente, Oakland, $\mathrm{CA} ;{ }^{2}$ Children's Hospitals and Clinics of Minnesota, Minneapolis, MN; ${ }^{3}$ HealthPartners, Minneapolis, $M N$

Background: Challenges in conducting prospective clinical research in the emergency department (ED) include 1) the unpredictable nature of the clinical environment, 2) the often short duration of patient stay, and 3) the difficulty in recognizing potentially eligible patients. To help mitigate these challenges and increase enrollment rates in ED research, we developed a software application that notifies physicians if their patients are eligible for enrollment in a prospective investigation. The Prospective Intelligence System for Clinical Emergency Services (PISCES) system scans electronic health records (EHR) for preselected clinical characteristics and reports to the treating physician via a real-time text message alert.

Methods: This investigation was conducted as part of a larger prospective study that implemented an EHR-based risk stratification tool for pediatric abdominal pain in the ED. PISCES alerts began 3 months into the data collection phase at 11 EDs across Northern California. When a patient, age 5-20 years, with a chief complaint of abdominal pain was admitted to the ED, the assigned provider received an automatic text alert on their mobile phone within 3 minutes. No protected health information was revealed in these text alerts. Other study recruitment techniques (site investigator education, iterative feedback, and gift card incentives) did not change across pre- and postintervention periods. Study enrollment rates were tracked before and after PISCES implementation on a monthly basis. We present aggregate enrollment over study periods by percentage of potentially eligible patients (patients who met basic eligibility criteria) before and after alert availability, and compare differences in their means via chi-squared and Fisher's exact tests.

Results: The study period ran from October 2016 through June 2017, with PISCES implementation in January 2017. Enrollment in the preintervention period was $10.2 \%$ (273 of 2687 eligible patients), with monthly rates ranging from $9.5 \%$ to $11.1 \%$. Enrollment in the postintervention period was $21.0 \%$ (1347 of 6424 eligible patients), with monthly rates ranging from $20.8 \%$ to $24.1 \%(\mathrm{P}<0.001)$.

Conclusion: Mobile phone text alerts of potential study candidates to emergency physicians significantly increased enrollment and can serve as a valuable tool to assist prospective research in the ED setting. Additional strategies are needed to further increase study enrollment. 
Keywords: information technology adoption and assessment, research administration, contracting, operations, dissemination, implementation, innovation

\section{OA1.04 \\ Connecting Communities With Data: A Practical Guide for Using Electronic Health Record Data to Support Community Health}

\section{Bree Allen, ${ }^{1}$ Karen Soderberg ${ }^{1}$ \\ ${ }^{I}$ Minnesota Department of Health, Saint Paul, MN}

Background: Minnesota's local health departments and hospitals have a common need to develop actionable, outcomes-oriented, and collaborative population health assessments. Many Minnesota hospitals, being newer to this reporting requirement, have reached out to their local public health (LPH) departments for support in developing these assessments and integrating social determinants of health. Data housed within hospitals' and health systems' electronic health record (EHR) systems have the potential to provide timely data for subpopulations, geographic areas, and health conditions that are typically underrepresented in traditional assessment methods.

Methods: We identified a number of collaborations including LPH departments, health plans, and providers that have developed in Minnesota for the purpose of sharing EHR data to support population health assessments. For example, the Private/Public Health Informatics Collaborative (PPHIC) is a partnership between multiple LPH departments within Hennepin County and 5 health systems serving residents in Hennepin County. The project's aim is to share EHR data from these providers, specifically around nonreportable diseases and conditions (eg, diabetes), to produce timely estimates of health status indicators to support program and policy evaluation. A team based at the Minnesota Department of Health interviewed these data-sharing collaborations to gather best practices and lessons learned, including definitions for EHR-extracted data, data-sharing agreement templates, integration of social determinants of health, data evaluation, and strategies for understanding data limitations.

Results: Practices and lessons from Minnesota collaborations have been combined into an informatics framework and toolkit for guidance to support any community to develop similar collaborations. The toolkit, freely available online, can be used by any community to support collaboration between LPH and health providers to explore the use of EHR-based data for community health assessments and public health prevention programs.

Conclusion: Communities face many challenges in acquiring and using actionable data to measure health outcomes. By working together to leverage EHR data and understand how these data can describe population health, LPH can develop strategies that can "move the needle" toward real outcomes and hospitals can understand how to apply meaningful strategies to support community health.

Keywords: rural health, information technology adoption and diffusion, primary care, state health policies, engagement of stakeholders, social determinants of health, health care organization, dissemination, implementation, innovation

\section{CHILD, MATERNAL, AND MENTAL HEALTH}

OA2.01

Risk of Febrile Seizures Following Measles-
Containing Vaccination in Children Born Preterm
or Full-Term

David McClure, ${ }^{1}$ Huong McLean, ${ }^{1}$ Nicola Klein,${ }^{2}$ Allison Naleway $^{3}$

${ }^{1}$ Marshfield Clinic Research Institute, Marshfield, WI; ${ }^{2}$ Kaiser Permanente Northern California, Oakland, CA; ${ }^{3}$ Kaiser Permanente Northwest, Portland, OR

Background: We examined whether the risk of seizures in children born preterm would alter the known risk relationship of age and exposure to measles-containing vaccines (ie, measles, mumps, rubella [MMR] or measles, mumps, rubella, varicella [MMRV]).

Methods: This was a Vaccine Safety Datalink cohort study of children who received MCV at age 12-23 months and were born from 2001 to 2014. Febrile seizures were identified by electronic diagnostic codes in the inpatient or emergency department setting. Using conditional Poisson regression, we estimated the incidence rate ratio (IRR) for seizures associated with measles-containing vaccine combinations ( $7-10$ days postvaccination exposure compared to 15-42 days unexposed).

Results: There were 532,375 children that received their first dose of MMR or MMRV at age 12 to 23 months; 45,343 were born preterm $(8.5 \%)$ and 487,032 were born full-term (91.5\%). Preterm children had similar IRRs compared with full-term children for any measles-containing vaccine administered (IRR [95\% CI]: 3.9 [2.5-6.1] vs 3.2 [2.7-3.7], respectively; interaction $\mathrm{P}=0.394$ ). The preterm birth status by vaccine exposure interactions stratified by age at vaccination were statistically indistinguishable $(\mathrm{P}=0.341$ and $\mathrm{P}=0.811$ for age 12-15 months and age 16-23 months, respectively). MMRV administered to preterm children 12-15 months old had a markedly larger IRR than for full-term children of the same age (12 vs 4.9), although the interaction P-value was modestly small (0.125).

Conclusion: Overall our results reinforce the recommendation to vaccinate preterm children at their chronologic age. The maximum benefit to risk of avoiding MMR and varicella disease for preterm children is to vaccinate according to the recommended childhood vaccine schedule.

Keywords: child and adolescent health, patient safety, epidemiology 
OA2.02

\section{Evaluating the Risk of Spontaneous Abortion Following Inadvertent Human Papillomavirus Vaccination in Pregnancy}

Elyse O. Kharbanda, ${ }^{1}$ Gabriela Vazquez-Benitez, ${ }^{1}$ Heather Lipkind, ${ }^{2}$ Sangini Sheth, ${ }^{2}$ Jingyi Zhu, ${ }^{1}$ Allison Naleway, ${ }^{3}$ Nicola Klein, ${ }^{3}$ Rulin Hecther, ${ }^{3}$ Matthew Daley, ${ }^{3}$ James Donahue, ${ }^{4}$ Michael Jackson, ${ }^{3}$ Alison Kawai, ${ }^{5}$ James D. Nordin ${ }^{1}$

${ }^{1}$ HealthPartners Institute, Minneapolis, $M N ;{ }^{2}$ Yale School of Medicine, New Haven, CT; ${ }^{3}$ Kaiser Permanente Northwest, Portland, OR; ${ }^{4}$ Marshfield Clinic Research Foundation, Marshfield, WI; ${ }^{5}$ Harvard Pilgrim Health Care Institute, Boston, $M A$

Background: The quadrivalent human papillomavirus vaccine (4vHPV) has been recommended for girls 9-26 years of age in the United States since June 2006. Although not intended for use in pregnancy, inadvertent exposures do occur. We evaluated the risk of spontaneous abortion (SAB) following $4 \mathrm{vHPV}$ vaccination during or peripregnancy within the Vaccine Safety Datalink.

Methods: Using data from 7 Vaccine Safety Datalink sites, we compared risks for $\mathrm{SAB}$ following $4 \mathrm{vHPV}$ in three exposure windows: distal (16-22 weeks prior to last menstrual period), peripregnancy (the 42 days prior to last menstrual period), and during pregnancy (up to 19 weeks gestation). Women 12-27 years of age with a pregnancy between 2008 and 2014 were required to have continuous insurance enrollment 8 months prior to pregnancy through the end of pregnancy. We excluded therapeutic abortions, ectopic pregnancies, gestational trophoblastic disease, multiple gestations, SABs before 6 weeks gestation, and women using medications increasing risk for SAB. Pregnancy outcomes were assessed using automated health care data for live births and via chart review for stillbirths and SABs. Two obstetricians performed case adjudication of SABs to confirm outcomes and gestational age at outcome. Risks for SAB following 4vHPV during pregnancy were compared to distal vaccination using timedependent covariate Cox models. Risks for SAB following peripregnancy versus distal vaccination were evaluated with standard Cox models.

Results: We identified 2800 pregnancies with 4vHPV exposure in a specified risk window: 33\% distal, 35\% peripregnancy, and $32 \%$ during pregnancy. Mean age was 22.4 years in distal and peripregnancy groups versus 21.4 years among women vaccinated during pregnancy. As compared to distal vaccination, women receiving 4vHPV during pregnancy had later entry into prenatal care (14 vs 12 weeks) and were more likely to receive other vaccines during pregnancy (43\% vs 24\%). Among 919 women with distal 4vHPV exposure, $10.4 \%$ experienced SAB. Receipt of $4 \mathrm{vHPV}$ during pregnancy or peripregnancy was not associated with increased risks for SAB (hazard ratios [95\% CI]: $1.10[0.81-1.51]$ and 1.07 [0.81-1.41], respectively).

Conclusion: Inadvertent 4vHPV exposure during pregnancy or peripregnancy is not associated with increased risk for SAB. Next steps are to evaluate risks for the 9-valent vaccine (9vHPV).

Keywords: child and adolescent health

\section{OA2.03}

\section{Longitudinal Trajectories of Antidepressant Use During Pregnancy and the Postnatal Period}

\author{
Gretchen Bandoli, ${ }^{1}$ Grace Kuo, ${ }^{1}$ Renu Sugathan, ${ }^{1}$ \\ Christina D. Chambers, ${ }^{1}$ Matthieu Rolland, ${ }^{2}$ Kristin \\ Palmsten ${ }^{3}$
}

${ }^{1}$ University of California, San Diego, San Diego, CA; ${ }^{2}$ University of Bordeaux, Bordeaux, France; ${ }^{3}$ HealthPartners Institute, Minneapolis, $M N$

Background: Studies of antidepressant safety in pregnancy typically reduce complex patterns of use into dichotomous variables. Herein, we use longitudinal trajectory modeling to describe patterns of antidepressant use in the first 32 weeks of pregnancy, and test whether these distinct trajectories are associated with a reduction in birth weight or gestational age at delivery.

Methods: Our study included 166 pregnant women with deliveries within the University of California San Diego Health system from 2011 to 2015 who were prescribed an antidepressant between 3 months prior to last menstrual period and 32 weeks of gestation. Using electronic medical records, we estimated average daily dose and cumulative dose per week for the first 32 weeks of gestation and for the first 13 weeks postnatal. We clustered women with similar utilization patterns using k-means longitudinal modeling and assessed the association between trajectory group and birth weight and gestational age at delivery.

Results: We identified four cumulative-dose trajectory groups and three average-daily-dose trajectory groups in each period. Relative to the lowest cumulative-dose trajectory group, the highest trajectory group during pregnancy was associated with reduced birth weight on multivariable analysis (average daily highest trajectory vs lowest trajectory $\beta$ : $-314.1 \mathrm{~g}$ [95\% CI: $-613.7,-15.5]$ ) adjusted for depression severity score, maternal age, race, and smoking in pregnancy. Trajectory groups were not associated with gestational age at delivery.

Conclusion: Trajectory groups of antidepressant use in pregnancy were associated with birth weight but not with gestational age at delivery. Longitudinal trajectories allow for a dynamic visualization and quantification of medication exposure during pregnancy.

Keywords: observational study, behavioral and mental health, epidemiology, pharmaceutical prescribing/costs 
OA2.04

Implementation of Screening, Brief Intervention, and Referral to Treatment for Behavioral Health Problems in Pediatrics: A Qualitative Study

Stacy Sterling, ${ }^{1}$ Ashley Jones, ${ }^{1}$ Esti Iturralde, ${ }^{1}$ Asheley Skinner, ${ }^{2}$ Agatha Hinman, ${ }^{1}$ Constance Weisner ${ }^{1}$

${ }^{1}$ Kaiser Permanente Northern California Division of Research, Oakland, CA; ${ }^{2}$ Duke University School of Medicine, Durham, NC

Background: Pediatric primary care is an opportune setting for screening, brief intervention, and referral to treatment (SBIRT), but it has not been widely implemented, and there is little research on factors contributing to its implementation. What literature there is has focused on implementation of SBIRT for adults.

Methods: This study used data from qualitative interviews $(\mathrm{N}=20)$ with Kaiser Permanente Northern California (KPNC) and community-based pediatric primary care clinicians, specialty mental health and substance abuse treatment clinicians, policymakers, and staff to examine factors that may inhibit or facilitate implementation of SBIRT in pediatric primary care. We used the Consolidated Framework for Implementation Research (CFIR) to inform our analysis, which used an inductive approach to build an understanding of the complexities involved in SBIRT implementation in pediatric primary care. Interviews were audiotaped, transcribed, and double-coded, independently, by coders blind to each other's coding, using NVivo software (QSR International Pty Ltd, Doncaster, Australia). Themes were created based on the broad constructs of the CFIR model: outer setting, inner setting, characteristics of the intervention, characteristics of the individuals involved, and process of implementation. Within those overarching constructs, SBIRT-specific subthemes were developed based on participant responses and informed by the extant literature. Percentage coder agreement and a Kappa coefficient were calculated to measure interrater reliability, by interview, node, and across the sample.

Results: Outer-setting factors such as confidentiality laws and societal attitudes about substance use also were frequently cited as influencing implementation, as were patient characteristics such as co-occurring mental health concerns. Many respondents discussed the role of clinician training and SBIRT skills. The single most frequently discussed implementation facilitator was adopting an integrated, embedded-behavioral health clinician model of SBIRT. Interrater reliability was high: Kappa coefficients ranged from 0.78 to 0.85 , and agreement between coders was $>98 \%$ across the constructs.

Conclusion: Participants identified many challenges to SBIRT implementation, but responses suggested a number of pragmatic policy and care delivery changes that could be taken to encourage its greater adoption. We discuss such facilitators in the context of real-world health systems.
Keywords: child and adolescent health, behavioral and mental health, substance abuse and addiction, dissemination, implementation, innovation

\section{HEALTH EQUITY}

OA3.01

Using Online Portals to Reach Underrepresented Primary Care Patients Due for Colorectal Cancer Screening: Opportunities and Challenges

Jennifer Elston Lafata, ${ }^{1}$ Amir Alishahitabriz, ${ }^{1}$ Patrice Jordan Fleming, ${ }^{1}$ Susan Flocke, ${ }^{2}$ Sarah Hawley, ${ }^{3}$ Resa Jones, ${ }^{4}$ Kenneth Resnicow, ${ }^{3}$ Deirdre Shires, ${ }^{5}$ Yongyun Shin, ${ }^{6}$ Shin-Ping Tu ${ }^{6}$

${ }^{1}$ University of North Carolina at Chapel Hill, Chapel Hill, $\mathrm{NC}$; ${ }^{2}$ Case Western Reserve University, Cleveland, OH; ${ }^{3}$ University of Michigan, Ann Arbor, MI; ${ }^{4}$ Temple University, Philadelphia, PA; ${ }^{5}$ Michigan State University, East Lansing, MI; ${ }^{6}$ Virginia Commonwealth University, Richmond, VA

Background: Patient portals represent an economical means to offer decision support, deliver behavior change interventions, and prevent loss to follow-up. We assess the ability to use an electronic health record's (EHR) patient portal to reach primary care patients and encourage colorectal cancer (CRC) screening following a physician order for screening.

Methods: Cohort of adults age 50-75 years due for CRC screening and receiving care from an integrated delivery system in southeast Michigan was identified $(\mathrm{N}=127,333)$. The EHR activity for those patients was electronically monitored for a physician order for a stool test or colonoscopy. Physician placement of the order triggered patients receiving a portal inbox message inviting them to explore an online CRC screening program. Using EHR data, we tracked when patients were sent an email, read the message, opened the attached communication, and agreed to study participation. Using multivariable logistic regression, we assessed race, gender, and age differences in account activation, message reading, program opening, and study participation agreement. Data are reported for the first 4 months of the trial (June 14, 2017-October 6, 2017).

Results: Overall, $59 \%(n=75,107)$ of patients did not have a portal account. Those with an account were significantly less likely to be male (odds ratio [OR]: $0.81,95 \%$ CI: $0.79-0.83$ ), black (OR: $0.48,95 \%$ CI: $0.47-0.49$ ) or other minority race (OR: $0.54,95 \%$ CI: $0.50-0.58$ ). During the initial trial period, a total of 1093 messages were sent. Recipients were $63.1 \%$ female, $62.5 \%$ white, $30.4 \%$ black, and $7.1 \%$ other race, and mean age was 59.4 (SD: 7.3); 71.5\% ( $\mathrm{n}=781)$ of recipients read the message, $34.5 \%(\mathrm{n}=377)$ opened the program, and $31.0 \%(n=339)$ agreed to study participation. Black patients were significantly less likely to read the message (OR: 0.48, 
95\% CI: 0.36-0.63). Likelihood of opening the program and agreeing to study participation increased with patient age (OR: 1.03, 95\% CI: 1.01-1.05 and OR: 1.03, 95\% CI: $1.01-$ 1.04 , respectively). No other significant differences in reach were found.

Conclusion: Findings illustrate opportunities and challenges afforded by online patient portals. Using the portal, we have been able to reach and enroll a relatively large number of primary care patients quickly and economically. Yet, black, male and younger patients are underrepresented among those we have reached and enrolled.

Keywords: cancer, survey research and methods, primary care, ambulatory/outpatient care, pragmatic trials, access to services

\section{OA3.02}

Measuring Health Equity in a Health Care System: Development of a Health Equity Index

\author{
Alice Pressman, ${ }^{1}$ Kristen Azar, $^{1}$ Alice Jacobson, ${ }^{1}$ \\ Ridhima Nerlekar, ${ }^{1}$ Sarah Robinson, ${ }^{1}$ Sylvia Sudat, ${ }^{1}$ \\ Elizabeth Dingbaum, ${ }^{1}$ Brendan Darsie, ${ }^{1}$ Janice \\ Rashed, ${ }^{1}$ Erika Hall, ${ }^{1}$ Maria Moreno, ${ }^{1}$ Stephen \\ Lockhart $^{1}$
}

\section{${ }^{1}$ Sutter Health Research, Development \& Dissemination, Walnut Creek, CA}

Background: Understanding health outcomes in socially or economically disadvantaged subgroups is crucial to improving community health, and it requires innovative analytics and dynamic application of clinical and population data. We designed and implemented a health equity index (HEI), a novel empirical approach to identify and prioritize health inequalities in health care systems.

Methods: The proposed HEI, an overall summary statistic, is also available by race category. It represents the average ratio of observed-to-expected hospital encounters for a given disease in a set timeframe at a given hospital within Sutter Health, a large health system in Northern California. Expected encounters for a condition are adjusted by age/ sex/race for underlying population distribution of hospital catchment area, condition prevalence, propensity to utilize the given hospital, and average frequency of that utilization. Underlying U.S. Census tract population estimates are derived from the American Community Survey. Prevalence estimates are derived from California's Behavioral Risk Factor Surveillance System and the California Health Interview Survey and compiled by the California Department of Public Health. Hospital utilization is calculated from Sutter Health's electronic health records. This presentation focuses on asthma at one hospital in Alameda County, California - Alta Bates Summit Medical Center (ABSMC). Results: In 2016, 649 patients utilized ABSMC 877 times for asthma. ABSMC's HEI of 1.5 indicates that among minority demographic subgroups, there were $50 \%$ more encounters than expected given the population characteristics. This was primarily driven by African American (AA) patients and, in particular, AA women age 60+ years (HEI: 2.3) and AA men age 45-64 years (HEI: 2.2). At this facility, utilization for asthma by Hispanics and non-Hispanic whites was less than expected across all ages, regardless of sex. After identifying our target population, we partnered with a local Federally Qualified Health Center to develop a pilot program for the population subgroup of AA asthma patients.

Conclusion: The HEI provides information to allow the health system to identify population subgroups that might benefit from patient-centered asthma intervention to reduce health inequities. The HEI and preliminary results from the asthma pilot will be presented.

Keywords: program evaluation, ambulatory/outpatient care, evaluation research, racial/ethnic differences, health care, social determinants of health, chronic disease, access to services, social work

\section{OA3.03 \\ Improving Thiazide Diuretic Dosing Among Blacks/ African Americans Using a Technology-Enabled Monitoring Tool: A Mixed-Methods Evaluation of a Health System Intervention}

\section{Cassondra Marshall, ${ }^{1}$ Alyce Adams, ${ }^{1}$ Lin Ma, ${ }^{1}$ Andrea Altschuler, ${ }^{1}$ Eileen Kim, ${ }^{1}$ Mark Kim, ${ }^{1}$ Nailah Thompson, ${ }^{1}$ Joseph Young ${ }^{1}$}

\section{${ }^{1}$ Kaiser Permanente Northern California, Oakland, CA}

Background: Despite national guidelines calling for use of thiazide diuretics as first-line therapy for blacks/ African Americans with hypertension, use of these clinically effective therapies is suboptimal. In 2015, Kaiser Permanente Northern California implemented a technologyenabled database monitoring tool to enable health care providers to identify black patients with poor blood pressure control who may benefit from thiazide diuretic initiation or intensification. We conducted a mixed-methods evaluation of this natural experiment.

Methods: We employed an interrupted time series design to determine if implementation of the tool led to an increase in the proportion of patients using any thiazide diuretics from 2012 to 2016. We modeled the month-to-month difference in thiazide use between blacks and whites, overall, and by gender. We also conducted key informant interviews with quality and operations leaders at 5 medical centers in the delivery system to identify modifiable facilitators and barriers to optimal thiazide dosing and implementation of the tool.

Results: We identified 425,379 eligible black $(n=49,305)$ and white $(n=269,415)$ patients. At baseline, $36.3 \%$ of all patients were using thiazide diuretics. A greater proportion of blacks used thiazides at baseline compared to whites, with highest use among black women (45.6\%) and lowest 
use among white women (39.3\%). The difference in thiazide use between black and white men increased immediately after implementation of the tool $(0.47$ percentage points, $\mathrm{P}<0.001$ ), reflecting an increase in the proportion of black men using thiazides postimplementation that was not present among whites. No difference was found for women. Factors affecting the tool's implementation included physician and pharmacist resistance to maximizing thiazide doses and differences in how and by whom the tool was managed, partially due to the proportion of blacks served by the medical center.

Conclusion: Implementation of the thiazide database monitoring tool led to an increase in the overall proportion of black men using thiazides, although the magnitude was small. Medical center-level variation in terms of how the tool was implemented and managed was substantial and contributed to the tool's effectiveness. Additional research is needed to examine the impact of the tool on thiazide dose intensification and to explore reasons for gender differences in thiazide use. Keywords: evaluation research, racial/ethnic differences, health, health care, qualitative research, quasi-experiments, chronic disease

\section{OA3.04}

Accessible Health Services Predict Community Health Center Patients' Mobile Health Technology Engagement: A Quantitative Cross-Sectional Analysis

\section{Sharon S. Laing, ${ }^{1}$ Ryan Sterling, ${ }^{2}$ Carlota Ocampo, ${ }^{3}$ Stacey Baugh ${ }^{3}$}

${ }^{1}$ University of Washington Tacoma, Tacoma, WA; ${ }^{2}$ University of Washington School of Public Health, Seattle, WA; ${ }^{3}$ Trinity Washington University, Washington, DC

Background: Mobile health technology (mHealth) can reduce health disparities by promoting health care involvement among low-income populations; studies are needed to ascertain factors likely to support an engaged patient, thus leading to positive health outcomes. Objectives: 1) assess mHealth knowledge and practices of community health center patients; and 2) evaluate health service factors predicting mHealth knowledge and practices.

Methods: Community health center patients in Washington state and Washington, DC, $(\mathrm{N}=159)$ completed a 47item, self-administered questionnaire. Primary outcomes: knowledge of smartphone use for wellness; frequency of smartphone use for wellness; and use of health apps. Predictors: 1) health services access (eg, health information access); 2) health services satisfaction; 3) healthy eating counseling; and 4) physical activity (PA) counseling. Multiple regression analyses assessed impact of predictors.

Results: Mean age was 34.98 years; study population comprised mostly women $(68 \%)$, white race $(36 \%)$, and income at/below \$20,000/year (63\%); 71\% reported knowledge of smartphone use for wellness, $73 \%$ used smartphones for wellness, and 48\% used health apps. Outcome 1: PA counseling contributed uniquely to smartphone use for wellness knowledge. Patients receiving PA counseling were 2.61 times more likely to know about using smartphones for wellness (odds ratio [OR]: 2.61, 95\% CI: $1.01-6.73 ; \mathrm{P}=0.047)$. Outcome 2: Two predictor variables made unique contributions to use of health-based mobile apps: PA counseling (OR: 2.89, 95\% CI: 1.17-7.17; $\mathrm{P}=0.022$ ), and access to health information (OR: 0.29 , 95\% CI: 0.09-0.96; $\mathrm{P}=0.043$ ). Respondents receiving PA counseling were 2.89 times more likely to report use of health-based mobile apps for wellness compared to individuals not receiving PA counseling. Respondents who reported having easy access to broad-based health information were 0.29 times less likely to report use of health-based mobile apps for wellness compared to those not having easy access to health information.

Conclusion: Results suggest that health service access at community health centers predicts patients' engagement with mobile health technologies outside of clinic settings.

Keywords: human subjects research, access to services, information technology, patient-provider communication, communication of research findings

\section{PAIN MANAGEMENT}

\section{OA4.01 \\ Impact of a Multidisciplinary Chronic Pain Management Program on Patient Care Utilization and Cost of Care}

Daniel Maeng, ${ }^{1}$ Kelly Baylor, ${ }^{1}$ John Han, ${ }^{1}$ John Bulger ${ }^{1}$

${ }^{1}$ Geisinger Health, Danville, PA

Background: In 2013, over 17,000 patients with chronic pain-related diagnoses were seen in Geisinger Clinic. Chronic pain patients also used over $\$ 5.1$ million in opioid prescriptions. In 2014, Geisinger's Department of Pain Medicine piloted the Multidisciplinary Pain Program (MPP) to address the needs of this population. MPP consisted of a 3-day seminar for each referred patient, followed by 12 months of comprehensive care with pain specialists. This study sought to examine the impact of MPP on care utilization and cost between 2014 and 2016.

Methods: This study was a retrospective health insurance claims data analysis covering a 3-year period from January 2013 through December 2016. Among all the patients who had been referred to MPP during the period, a subset of those who were also Geisinger Health Plan (GHP) members was identified. Those patients who were referred to MPP after December 2016 served as the contemporaneous comparison 
group. GHP's claims data for the corresponding period were analyzed on a per-member-per-month basis. A multivariate linear regression model with patient fixed effects was estimated for each of the following dependent variables: total cost (medical + prescription drug), frequencies of acute hospital admission, outpatient emergency department visits, and physician office visits, as well as frequency of high-end diagnostic imaging (CT, MRI, PET, etc).

Results: 113 patients were included in the MPP intervention group, and 69 were included in the comparison group. The MPP intervention group was followed, on average, for approximately 1 year after the initial MPP appointment. MPP was associated with $42 \%$ reduction in acute hospitalization $(\mathrm{P}=0.029), 18 \%$ reduction in primary care office visits $(\mathrm{P}=0.008)$, and $38 \%$ reduction in high-end imaging utilization $(\mathrm{P}=0.013)$. MPP also was associated with $23 \%$ increase in specialist visits $(\mathrm{P}=0.038)$ but was not associated with outpatient emergency department visits. Lastly, MPP was associated with $20 \%$ total cost of care reduction $(\mathrm{P}=0.038)$.

Conclusion: MPP has been designed to improve function and quality of life for individuals living with chronic pain. Results of this study support this expectation by demonstrating that MPP was associated with significantly lower care utilization and cost.

Keywords: financial analysis, ambulatory/outpatient care, evaluation research, complex disease management, multiple chronic conditions, chronic disease, health care costs, resource use

\section{OA4.02}

\section{Doctor-Patient Trust Among Chronic Pain Patients on Chronic Opioid Therapy: A Survey}

Karen Sherman, ${ }^{1}$ Rod Walker, ${ }^{1}$ Kathleen Saunders, ${ }^{1}$ Susan Shortreed, ${ }^{1}$ Michael Parchman, ${ }^{1}$ Ryan Hansen, ${ }^{2}$ Manu Thakral, ${ }^{1}$ Evette Ludman,, ${ }^{1}$ Sascha Dublin, ${ }^{1}$ Michael Von Korff ${ }^{1}$

${ }^{1}$ Kaiser Permanente Washington, Seattle, WA; ${ }^{2}$ University of Washington, Seattle, WA

Background: Risk reduction initiatives for patients on chronic opioid therapy (COT) are desirable to prevent known harms. However, more active and structured monitoring, key components in many plans, may have adverse effects on already challenging doctor-patient relationships. This analysis examined the level of trust in the doctor-patient relationship among COT patients exposed to an opioid dose-reduction and risk-stratification/monitoring initiative compared to patients who were not.

Methods: We surveyed 1588 adults with chronic pain receiving COT about their views of trust in their doctor, their doctor's trust in them, and their concerns about opioid prescribing and safety of opioids. The population included adults receiving care in intervention settings that implemented opioid dose-reduction and risk-stratification/ monitoring initiatives $(n=653)$ and control settings with similar COT patients that did not $(\mathrm{n}=935)$.

Results: Among COT patients, $86.1 \%$ in the control clinics trusted their doctor's judgment compared to $77.9 \%$ in the intervention clinics $(\mathrm{P}=0.002)$. The percentage who worried that their doctor would stop prescribing opioid pain medicine was $29.3 \%$ in intervention clinics and $21.8 \%$ in control clinics $(\mathrm{P}=0.007)$. Among COT patients, $5.8 \%$ from intervention clinics compared to $3.2 \%$ from control clinics did not believe that their physician trusted in how they managed their opiate pain medicines $(\mathrm{P}=0.002)$.

Conclusion: While COT patients typically reported favorable perceptions of clinician-patient trust in managing opiate pain medicines, implementation of dose-reduction and risk-stratification/monitoring initiatives may have reduced levels of trust for a small percentage of COT patients. This is consistent with prior research suggesting that efforts to reduce COT dose and to more closely monitor opiate use can cause stress in doctor-patient relationship.

Keywords: program evaluation, quality of care, patientprovider communication, clinical decision-making, chronic disease, quality improvement, patient experience/ satisfaction

\section{OA4.03}

Impact of an Opioid Prescribing Protocol on the Prescribing Behavior at the University of Minnesota School of Dentistry

\author{
Harold Tu, ${ }^{1}$ Robert Nadeau, ${ }^{2}$ Chad Wagner ${ }^{2}$ \\ ${ }^{1}$ University of Minnesota School of Dentistry, Minneapolis, \\ $M N ;{ }^{2}$ University of Minnesota, Minneapolis, $M N$
}

Background: This study evaluates the use of opioid analgesics at the University of Minnesota School of Dentistry prior to and following implementation of an opioid prescribing protocol for acute postsurgical pain. The misuse of prescription pain relievers is the second most common form of drug abuse behind marijuana. Dentists are among the leading opioid prescribers and are responsible for the majority of prescriptions to minors. To improve postoperative pain management and limit the misuse of opioid medication, the university's Division of Oral and Maxillofacial Surgery introduced an opioid prescription protocol in February 2016. The protocol was based on evidence that acute postoperative pain can be effectively managed utilizing nonsteroidal antiinflammatory drugs as the first pain management drug of choice.

Methods: Retrospective analysis compares the number of total opioid prescriptions and the number of tablets per prescription in the previous 10 quarters prescribed by the University of Minnesota School of Dentistry. Prescription data were collected from institutional electronic health record software.

Results: The results show an overall $47.1 \%$ decrease in the 
total number of opioid prescriptions in the 5 quarters after the initial opioid prescribing protocol was approved by the Clinical Affairs Committee in February 2016.

Conclusion: These results support the hypothesis that an opioid prescribing protocol would be successful in decreasing the total number of prescriptions written and number of tablets dispensed per prescription.

Keywords: substance abuse and addiction, clinical decisionmaking, dental care, quality improvement, pharmaceutical prescribing/costs

\section{OA4.04}

\section{Transformative Self-Management for Chronic Pain Utilizing Online Training and Telehealth Coaching}

James Fricton, ${ }^{1}$ Robin Whitebird, ${ }^{2}$ Gabriela VazquezBenitez, ${ }^{1}$ Jeanette Y. Ziegenfuss, ${ }^{1}$ Elizabeth Grossman ${ }^{1}$

${ }^{1}$ HealthPartners Institute, Minneapolis, $M N ;{ }^{2}$ University of St. Thomas, Minneapolis, $M N$

Background: The U.S. Institute of Medicine has made research on pain conditions among its highest priorities due to their high prevalence, functional limitations, missed work, opioid dependency, and high cost. They recommend that health professionals' primary role for chronic pain should be guiding, coaching, and assisting patients with day-to-day self-management. However, health professionals often lack the time and training to perform this role, and there has been limited research on approaches for selfmanagement. A transformative care model integrates robust self-management training with evidence-based treatment to improve outcomes of care. The purpose of this research was to evaluate a telehealth coach-based online training program for self-management of chronic pain as part of routine care. Methods: The Personalized Activated Care and Training (PACT) program was developed to enhance understanding, compliance, and success in self-management of pain conditions. The program provides patients with a telehealth coach and self-directed online self-management modules using cognitive behavioral strategies to train patients to reduce risk factors that contribute to delayed recovery and implement protective actions that can reduce chronic pain. We conducted a pilot randomized clinical trial to refine the program and evaluate the feasibility to conduct a full trial. We compared the PACT program to traditional self-care (TSC) for temporomandibular disorder pain. All participants continued usual treatment with their dental providers. We evaluated preliminary efficacy on mean change of pain severity, jaw function, and pain interference with daily living at 8 weeks, presented as Cohen's $d$ statistics.

Results: A total of 81 subjects were randomized to PACT or TSC; one subject withdrew from the study. Mean age was 47 years (SD: 16), 86\% of subjects were female, and $82 \%$ had pain onset of 1 year or more. Participants showed improvement in measures of pre-post change at 8 weeks for pain severity (PACT: -0.94 vs TSC: -0.5 ) but not for pain interference or jaw functioning.

Conclusion: This research demonstrated that a transformative care model including telehealth coaching and online training was feasible and ready for a broader clinical trial. It provides an accessible, scalable, and transferable method for self-management that can be integrated into routine care without increasing the burden of time demands on providers.

Keywords: information technology, patient-provider communication, patient experience/satisfaction

\section{INFORMED DECISION-MAKING}

\section{OA5.01}

What Happens if Things Go Wrong? Searching for Evidence of Preoperative Advance Care Planning Discussion and Documentation Among Older Adults Undergoing High-Risk Surgery

Ellis Dillon, ${ }^{1}$ Yan Yang, ${ }^{1}$ Victoria Tang, ${ }^{2}$ Rebecca Sudore, ${ }^{2}$ Ming Tai-Seale ${ }^{3}$

${ }^{1}$ Palo Alto Medical Foundation Research Institute, Mountain View, CA; ${ }^{2}$ University of California, San Francisco, San Francisco, CA; ${ }^{3}$ University of California, San Diego, San Diego, CA

Background: Older adults with multiple comorbid conditions undergoing high-risk surgery are likely to suffer life-altering complications, which often lead to functional and/or cognitive decline and loss of independence. Experts strongly recommend advance care planning (ACP) in the preoperative setting. It is unknown whether ACP discussions happen prior to high-risk surgeries or if they are documented in the electronic health record (EHR).

Methods: Data were from a multispecialty group practice extracted from January 2013 to December 2014. Patients included were $\geq 65$ years old, had $\geq 1$ office visit and a Charlson index score $\geq 2$, and underwent high-risk surgery (defined by CPT codes associated with $\geq 1 \%$ postoperative 30-day or in-hospitalization mortality rate). Preoperative documentation of ACP was defined as a Physician Order for Life-Sustaining Treatment (POLST) or advance directive in the EHR's problem list. Logistic regression was performed to test associations between $\mathrm{ACP}$ documentation and patient characteristics. Detailed chart review of a random sample of 25 decedents assessed the presence and extent of ACP discussion and documentation.

Results: 393 patients were identified with high-risk surgeries. Their mean age was 79 years, $45 \%$ were female, $26 \%$ were nonwhite, and 26\% $(\mathrm{n}=101)$ had ACP documented prior to surgery. Older age (ie, $85+$ years, $\mathrm{P}<0.05$ ), being white $(\mathrm{P}<0.05)$, having $\geq 4$ office visits in the year prior to surgery $(\mathrm{P}<0.001)$, and having dementia/mild cognitive 
impairment $(\mathrm{P}<0.01)$ were associated with higher odds of preoperative ACP documentation. Chart review revealed that most documentation of ACP in progress notes was either absent or cursory and did not include goals of care or values, eg, "Patient does not want CPR," "Brought me a copy of advance directive, discussed," "Feels strongly that does not want to have CPR or ventilator."

Conclusion: Only a quarter of older high-risk surgical patients with multimorbidity had ACP documentation in the EHR. Nonwhite patients were less likely and older patients and those with cognitive impairment were more likely to have ACP documentation. Chart review demonstrated that documentation of these wishes may be sparse. ACP should be incorporated into the preoperative workflow and should include documented goals of care and values in addition to legal forms.

Keywords: aging, elderly, geriatrics, end-of-life care/ decisions, patient-provider communication, ambulatory/ outpatient care, complex disease management, multiple chronic conditions

\section{OA5.02}

\section{A Randomized Controlled Trial of Emergency Department Dental Care Vouchers to Improve Care and Reduce Return Visits}

\author{
Bjorn Westgard, ${ }^{1}$ Kory Kaye, ${ }^{1}$ Abigail Zagar, ${ }^{1}$ Jeffrey \\ Anderson, ${ }^{1}$ Sandi Wewerka, ${ }^{1}$ Kevin Nakagaki ${ }^{1}$
}

\section{${ }^{1}$ HealthPartners, Minneapolis, $M N$}

Background: Emergency department (ED) visits are increasing everywhere, with $1.4 \%$ of visits related to nontraumatic dental conditions (NTDC). These visits are associated with high return rates due to the limited interventions available in the ED, widespread lack of dental insurance, and difficulties accessing definitive dental care. Current standards of care lead to treatment dissatisfaction and provider stress and are frequently ineffective, insufficient, and systemically costly.

Methods: We conducted a randomized controlled trial to determine if distributing a voucher for prompt dental care decreases return rates to the ED for patients with NTDC who have Medicaid or no dental insurance. We asked providers to predict which of these patients would follow up with a dentist and utilize their vouchers. We also compared actual treatment costs for participants' visits to the ED and at our collaborating dental clinic.

Results: From 2015 to 2016, we randomized 316 participants to voucher $(n=129)$ or no-voucher $(n=187)$. In intention-totreat analysis, voucher recipients had a significantly lower likelihood of returning to the ED at 30 days (odds ratio: 0.48 , 95\% CI: $0.22-1.02)$ and 90 days (0.49, 0.25-0.96) as well as a longer time to first return to the ED (0.50, $0.24-1.02 ; 0.51,0.27-0.97)$. We found no confounding associations with demographics (age, sex, race), treatments given in the ED (antibiotics, dental block, narcotics), insurance status, voucher assignment and redemption, and ED returns. Provider prediction of voucher redemption had poor positive and fair negative predictive values $(0.531$ and 0.753 , respectively). We found no significant difference in total ED charges or net margins between the two groups. Of note, $49 \%$ of dental vouchers were redeemed, covering an average of $79 \%$ of clinic costs.

Conclusion: Our study found that vouchers for prompt dental care can provide access to definitive care for NTDC and reduce ED return visits without changing ED margins. Providers are poor predictors of patient follow-up with dentistry. Though vouchers covered the majority of dental clinic costs, the average cost of a clinic visit for definitive care was less than the average cost of an ED visit for temporizing care. To improve patient access to appropriate care, health system coordination and cost sharing between EDs and dental clinics should be explored further.

Keywords: health care costs, resource use, access to services, dental care

OA5.03

Precision Pharmacy: Positioning Clinical Pharmacists to Improve Patient Care Through Genetically Guided Medication Selection

Rebecca Pulk, ${ }^{1}$ Mike Evans, ${ }^{1}$ Jenna Carmichael, ${ }^{1}$ Gerald Greskovic, ${ }^{1}$ Melissa Kern, ${ }^{1}$ Dean Parry, ${ }^{1}$ Eric Wright, ${ }^{1}$ Marc Williams ${ }^{1}$

\section{${ }^{1}$ Geisinger Health, Danville, PA}

Background: Precision medicine promises to improve patient care through the consideration of the whole person, including genetics. We introduce the concept of "precision pharmacy," in which pharmacists are empowered to implement precision medicine through the integration of genetic information into medication selection, dosing, and monitoring. This triad conceptual model consists of applied clinical pharmacogenomics, medication management for patients with genetically identified disease, and targeted cancer pharmacotherapy.

Methods: We describe the conceptual model of precision pharmacy with select examples from the implementation throughout Geisinger Health.

Results: Applied clinical pharmacogenomics matches genomic test results to just-in-time medication use and is built on testing, data storage, clinical decision support, and consultation. Through the genomic pharmacy consultation service, the integration of pharmacogenomic-guided therapy selection is occurring across Geisinger. This service is facilitating the return of preemptive pharmacogenomic panel results for a cohort of 2500 patients, with integrated clinical decision support within the medical record. This service has partnered with Geisinger's MyCode return of results team to guide medication therapy in patients with pathogenic/likely 
pathogenic variants associated with inherited disease. To date, pharmacists have cared for over 100 patients receiving genetic results, performing comprehensive medication reviews, providing recommendations on potential chemoprevention and medication avoidance directly to patients and providers, documenting findings in the medical record, and referring patients to Geisinger's Medication Therapy Disease Management Program pharmacists for "inperson" clinic visits for conditions for which collaborative practice agreements are in place. Finally, targeted cancer pharmacotherapy matches chemotherapy to tumor-specific genetic markers. Oral chemotherapy pharmacists play an active role in the management of targeted chemotherapeutic regimens tailored to the unique genetic fingerprints within each patient's malignancy. This team currently manages a panel of 800 patients receiving targeted agents.

Conclusion: As precision medicine evolves and genetic testing becomes more common, pharmacists are key members of the multidisciplinary team needed to improve the care and outcomes of patients. Precision pharmacy as implemented within Geisinger can serve as a comprehensive strategy that guides health systems, practitioners, payers, and patients in the most effective use of genomic information to optimize medication therapy. As medication experts, pharmacists are positioned to lead this effort.

Keywords: cardiovascular disease, cancer, pharmacy, ambulatory/outpatient care, dissemination, implementation, innovation, patient safety, pharmaceutical prescribing/costs

\section{OA5.04}

We Want to Know: Patient Comfort Speaking Up About Breakdowns in Care and Patient Experience

Kimberly A. Fisher, ${ }^{1}$ Kelly Smith, ${ }^{2}$ Thomas H. Gallagher, ${ }^{3}$ Jim Huang, ${ }^{2}$ Jim Borton, ${ }^{4}$ Kathleen M. Mazor ${ }^{1}$

${ }^{1}$ University of Massachusetts Medical School / Meyers Primary Care Institute, Worcester, MA; ${ }^{2}$ MedStar Health Research Institute, Hyattsville, MD; ${ }^{3}$ University of Washington, Seattle, WA; ${ }^{4}$ MedStar Health, Columbia, MD

Background: Patients have important insights into care experiences, particularly about breakdowns in care. However, many patients are reluctant to speak up about breakdowns, resulting in missed opportunities to improve care. We sought to identify patient characteristics associated with speaking up and to examine whether speaking up is associated with better patient experiences.

Methods: We created a new item: "How often did you feel comfortable speaking up if you had any problems in your care?" with the following response options: always, usually, sometimes, never, and Did not have any problems. This item was administered as part of the Hospital Consumer Assessment of Healthcare Providers and Systems (HCAHPS) patient satisfaction survey at 8 hospitals between May 2016 and April 2017. Respondents were grouped as follows: 1) no problems during hospitalization; 2) always felt comfortable speaking up; and 3) usually/sometimes/never felt comfortable speaking up. We compared these groups for demographics and selected patient experience items using chi-squared and Student's t-test. A binomial logistic regression model was constructed to identify independent predictors associated with not experiencing problems and always feeling comfortable speaking up.

Results: Responses from 10,212 patients were analyzed: 5254 indicated no problems; 3444 always felt comfortable speaking up; 1514 usually/sometimes/never felt comfortable speaking up. Patients who did not have any problems were older, more likely to be African American or Hispanic, less likely to be college educated, and reported better overall and mental health than patients who experienced a problem. Among patients who experienced a problem, patients who were always comfortable speaking up were younger, more likely to speak English, and reported better overall and mental health compared to patients who were not always comfortable speaking up. Patients who always felt comfortable speaking up reported better nurse communication ( 80.4 vs $47.8 ; \mathrm{P}<0.01)$ and better physician communication ( 82.6 vs $57.2 ; \mathrm{P}<0.01$ ), provided higher hospital ratings (8.7 vs $7.1 ; \mathrm{P}<0.01)$, and were more likely to definitely recommend the hospital $(71.7 \%$ vs $36.7 \%$; $\mathrm{P}<0.001)$ than patients who were not always comfortable speaking up.

Conclusion: Patients who are always comfortable speaking up about problems reported better experiences of care across multiple HCAHPS dimensions. This illustrates the importance of encouraging patients to speak up about problems in care, as these may be opportunities to address breakdowns and improve patients' experience of care.

Keywords: patient-provider communication, hospital, patient safety, patient experience/satisfaction

\section{HEALTH SYSTEM IMPROVEMENT}

\section{OA6.01}

\section{Sustained Impact of Lean Redesigns on Primary Care Workflow Efficiency}

Dorothy Hung, ${ }^{1}$ Quan Truong, ${ }^{1}$ Qianyun Zhang, ${ }^{1}$ SuYing Liang, ${ }^{1}$ Harold S. Luft ${ }^{1}$

\section{${ }^{1}$ Palo Alto Medical Foundation Research Institute, Mountain View, CA}

Background: Workflow interventions are increasingly being implemented to enhance practice efficiency. We examined the sustained impact of Lean redesigns on physician workflows 3.5 to 5 years after being introduced in primary care clinics. We monitored performance metrics 
before and after redesigns were implemented in 46 internal medicine, family medicine, and pediatrics departments at a large ambulatory care delivery system.

Methods: Using a stepped-wedge study design with interrupted time series analysis, we examined four workflow metrics reflecting physicians' timely completion of tasks: 1) closure of patient charts $<2$ hours of the office visit; 2) electronic reply to patient messages; 3 ) renewal of prescription medications; and 4) resolution of telephone calls, each completed in $<4$ business hours. Using generalized linear mixed models, we first estimated the immediate impact of Lean on physician workflows. We then estimated these effects over time by comparing postintervention metrics with projected values had Lean not been implemented. These counterfactual values were projected by extending baseline trends in workflow forward until the end of the study period. 95\% bias-corrected bootstrap confidence intervals were used to compare differences between post-Lean intervention and projected values absent Lean.

Results: After Lean intervention, there were significant increases in timely office visit chart closures $(+27.7 \%)$, telephone resolutions $(+14.1 \%)$, and prescription refills $(+34.9 \%)$. When comparing differences post-Lean vs projected values over time, we found that physicians did better at meeting targets for responding to patient messages (84.3\% completion) and refilling prescriptions (59.1\%) with Lean redesigns as compared with projected metrics absent Lean $(80.0 \%$ and $53.5 \%$, respectively). However, timely chart closure (55.3\%) and telephone response (55.0\%) after the redesigns were slightly lower over time compared with projected values at the end of the study period $(56.6 \%$ and $57.8 \%$ timely completion rates, respectively).

Conclusion: These findings align with previous research showing Lean redesigns can positively impact workflow efficiency. However, despite large improvements immediately following implementation, after several years we found attenuated effects in some metrics. This finding raises questions about intervention sustainability and longer-term impacts of Lean redesigns on physician tasks and workflows.

Keywords: health care workforce, primary care, quality improvement

\section{OA6.02}

\section{Referrals and Diagnostic Orders as a Tool to Conclude Primary Care Visits Running Over Time or Behind the Schedule}

\author{
Sukyung Chung, ${ }^{1}$ Harold S. Luft ${ }^{1}$ \\ ${ }^{1}$ Palo Alto Medical Foundation Research Institute, Palo \\ Alto, $C A$
}

Background: Time is the most scarce commodity in primary care practices. While it is expected that visits with patients who have complex conditions or who present new problems may take extra time, primary care providers (PCPs) need to conclude a visit within a reasonable time frame in order to be on time for following appointments. With time pressure, providers may consider tradeoffs between time and other resources including referrals or diagnostic procedures. We sought to understand situations when PCPs spend additional time with patients. We then assessed whether PCPs resort to more diagnostic orders or referrals when facing time constraints.

Methods: Routine nonpreventive primary care visits of established adult patients in a large group practice organization were studied. Data from electronic health records (EHR) were used to create indicators of patient, provider, and practice characteristics at each visit. Access logs of EHR were used to estimate visit length, defined as time between a PCP first logged in and last logged out of the patient chart from the examination room computer. Visits with valid access log data, in addition to other characteristics, in $2014(\mathrm{~N}=339,947)$ were analyzed using generalized linear models with provider random effects.

Results: Visits were longer for patients who were older, had multiple chronic conditions, and were the PCP's own patients. Visits were also longer when new problems were noted or procedures were done. Given patient and visit characteristics, PCPs spent less time when they were under time pressure such that visits were significantly shorter when providers were running behind schedule and were longer when the visit was followed by a break. Part-time physicians spent more time with each patient. The longer predicted visit length based on these factors was the more likely the visit was to be involved with a referral, lab, or imaging order. After controlling for the predicted visit length, PCPs were much more likely to make diagnostic orders or referrals when actual visit length was longer than predicted visit length.

Conclusion: Primary care physicians adjust visit length under time pressure. Making diagnostic orders or referrals may be a way to conclude a visit when it stretches beyond expected time.

Keywords: observational study, primary care, ambulatory/ outpatient care, health care costs, resource use, access to services, incentives

\section{OA6.03 \\ Factors Associated With Physician Well-Being - Time for a Change}

Ming Tai-Seale,,${ }^{1,2}$ Robert Nordgren, ${ }^{2}$ Yan Yang, ${ }^{2}$ Amy Meehan, ${ }^{2}$ Ruth Steinberg, ${ }^{2}$ Jin Chang, ${ }^{2}$ Albert Chan, ${ }^{2}$ Ellis Dillon, ${ }^{2}$ Jinnan Li, ${ }^{2}$ Cliff Olson, ${ }^{2}$ Tim Lee, ${ }^{2}$ Teresa Nauenberg, ${ }^{2}$ Susan Connolly, ${ }^{2}$ Dominick Frosch ${ }^{2}$

${ }^{1}$ University of California, San Diego, San Diego, CA, ${ }^{2}$ Palo Alto Medical Foundation, Sutter Health, Palo Alto, CA 
Background: About half of America's physicians report burnout symptoms. In response to an organizational priority to enhance physician well-being, we assessed physician well-being and empirically examined its relationship with factors encompassing multiple dimensions of their work and life.

Methods: The study took place in a large multispecialty group practice serving about 1 million patients. A survey was delivered to 1292 employed physicians regarding life satisfaction, burnout symptoms (using a nonproprietary single-item measure), self-care practices, and perceptions of work environment. Survey responses, electronic health record, and administrative data on physician workload, eg, number of InBasket messages received per week, clinical FTE, and leadership role, were used in descriptive and multivariate regression analyses.

Results: Survey response rate was $72 \%$. Average life satisfaction was 78 (SD: 16) on a scale of $0-100 ; 36 \%$ reported burnout symptoms, 36\% slept $\geq 7$ hours/ night, $70 \%$ exercised $\geq 2$ days/week, $31 \%$ practiced mindfulness $\geq 1$ day/week; $83 \%$ perceived having control over work schedule; $12 \%$ felt that physicians are highly valued; $60 \%$ reported calm atmosphere in work area. In terms of workload, $43 \%$ received more than the average (ie, 183) number of InBasket messages per week. Life satisfaction was positively and significantly associated with self-care practices such as sleeping $\geq 7$ hours/night ( $\mathrm{P}<0.001)$, exercising $\geq 2$ days/week $(\mathrm{P}<0.001)$, practicing mindfulness $\geq 1$ day/week $(\mathrm{P}<0.01)$, perceived control over work schedule $(\mathrm{P}<0.001)$, feeling highly valued $(\mathrm{P}<0.01)$, and a positive atmosphere in work area $(\mathrm{P}<0.01)$. Female physicians reported lower life satisfaction $(\mathrm{P}<0.05)$ and higher odds of burnout $(\mathrm{P}<0.05)$. Higher odds of burnout also were associated significantly with higher volume of InBasket messages $(\geq 183)$ received per week $(\mathrm{P}<0.01)$ and with having a leadership role $(\mathrm{P}<0.05)$. Physicians sleeping $\geq 7$ hours/night $(\mathrm{P}<0.001)$, exercising $\geq 2$ days/ week $(\mathrm{P}<0.01)$, perceiving control over work schedule ( $\mathrm{P}<0.001)$, feeling highly valued $(\mathrm{P}<0.001)$, and reporting calm atmosphere in work area $(\mathrm{P}<0.001)$ had lower odds of burnout.

Conclusion: Life satisfaction and burnout symptoms varied by self-care practices, InBasket message volume, gender, leadership role, and perceived work experience, suggesting important protective and harmful associations with physician well-being. Changes in health care have resulted in increasing numbers of female physicians, physicians with leadership roles, and proliferating InBasket messages. Organizational changes are necessary to address these risk factors, eg, reducing/restructuring InBasket work and better supporting leaders and female physicians. Keywords: health care workforce, observational study, survey research and methods
OA6.04

Using Boot Camp Translation to Inform a SystemBased Intervention to Improve Rates of Colon Cancer Screening Among Latino Patients in Community Health Centers

Jamie Thompson, ${ }^{1}$ Melinda Davis, ${ }^{2}$ LeAnn Michaels, ${ }^{2}$ Jennifer Rivelli, ${ }^{1}$ Marta Castro, ${ }^{3}$ Brittany Younger, ${ }^{3}$ Melissa Castillo, ${ }^{3}$ Sacha Reich, ${ }^{1}$ Gloria Coronado ${ }^{1}$

${ }^{1}$ Kaiser Permanente Center for Health Research, Portland, OR; ${ }^{2}$ Oregon Health \& Science University, Portland, OR; ${ }^{3}$ AltaMed Health Services, Los Angeles, CA

Background: Colon cancer is the second-leading cause of cancer death in the United States, and screening rates are disproportionately low among Latinos. One factor thought to contribute to the low screening rate is the difficulty Latinos encounter in understanding health information and therefore in taking appropriate health action. As part of our study, we used boot camp translation, a community-based participatory approach, to elicit input from diverse stakeholders (ie, patients, clinic staff) and refine the messages and format of colon cancer screening reminders for a direct mail fecal immunochemical testing (FIT) program.

Methods: Patient participants were Latino, age $50-75$ years, able to speak English or Spanish, and willing to participate in an in-person meeting and follow-up conference calls. Separate sessions were held for English- and Spanishspeaking participants. As part of the in-person meetings, a bilingual colon cancer expert delivered a presentation on colon health and screening messages. Boot camp translation facilitators led interactive sessions where participants reviewed materials and reminder messages using various modalities (eg, text, automated call recordings). We asked participants to consider what information about colon cancer screening is important, what the best methods are to share these messages, and the timing and frequency with which these messages should be delivered to patients to encourage FIT completion. Results were used to define the intervention for the pilot and main trial.

Results: A total of 25 adults participated in the in-person sessions (English, $\mathrm{n}=12$; Spanish, $\mathrm{n}=13$ ). Patient participants were primarily enrolled in Medicaid/uninsured (64\%) and had annual household incomes less than $\$ 20,000$ (56\%). Key themes from the process included increasing awareness about colon cancer screening, stressing the urgency of screening, enhancing discussions between provider and patient, and using personalized messages in reminders. Both groups noted the importance of receiving an alert before the FIT is mailed and a reminder within 2 weeks of FIT mailing. Conclusion: Using boot camp translation, we successfully incorporated patient feedback to design culturally relevant health messages to promote FIT testing among Latino patients served by community clinics.

Keywords: cancer, patient-provider communication, racial/ ethnic differences, health, health care 


\section{CARDIOVASCULAR DISEASE}

\section{OA7.01 \\ Bleeding and Thromboembolism Associated With CYP3A4 Inhibitors in Patients on Rivaroxaban}

Joseph Goble, ${ }^{1}$ Kiumars Zolfaghari, ${ }^{2}$ Stephanie Yu, ${ }^{1}$ Paul Godley, ${ }^{1}$ Laurel Copeland, ${ }^{2}$ Gregory Dehmer, ${ }^{3}$ Jeffrey Michel ${ }^{3}$

${ }^{1}$ Department of Pharmacy, Baylor Scott \& White Health, Temple, TX; ${ }^{2}$ Center for Applied Health Research, Baylor Scott \& White Health, Temple, TX; ${ }^{3}$ Division of Cardiology, Baylor Scott \& White Health, Temple, TX

Background: The factor Xa inhibitor rivaroxaban is the most frequently prescribed novel oral anticoagulant (NOAC) in our health care system. It is both metabolized via cytochrome P450 3A4 (CYP3A4) and undergoes renal excretion, with potential for CYP3A4 inhibitors (CYP3A4-i) to alter serum levels and clinical activity. Increased bleeding is known to occur with coadministration of platelet aggregation inhibitors (PAI). We hypothesized that coadministration of CYP3A4-i might increase bleeding risk while reducing thromboembolism.

Methods: Claims from a 250,000-member system generated a patient cohort $\geq 18$ years of age with $\geq 1$ claim for rivaroxaban during 2012-2015. Inclusion required documented prescriptions filled for rivaroxaban and CYP3A4-i based on pharmacy claims data. Exclusions were $>3$-month gap in health plan enrollment, and no medical claims pre/post-rivaroxaban administration. Coadministrated CYP3A4-i included clarithromycin, diltiazem, dronedarone, erythromycin, and verapamil. PAI included anagrelide, aspirin, cilostazol, clopidogrel, and ticagrelor. Patients were designated as receiving CYP3A4-i or receiving PAI, leaving the comparison group on rivaroxaban alone (rivaroxabanonly).

Results: A total of 751 patients with average age of 70.8 years (50\% male) were included. Compared to rivaroxaban-only, patients given CYP3A4-i (18.4\%) were older (74.7 vs 69.9, $\mathrm{P}<0.0001)$ and more likely to have atrial fibrillation $(87 \%$ vs $47 \%, \mathrm{P}<0.0001$ ). Bleeding occurred in $8.1 \%$ of all patients on rivaroxaban, but more commonly with coadministration of CYP3A4-i $(13.8 \%$ vs $6.9 \%, \mathrm{P}=0.007)$ or PAI $(17.5 \%$ vs $7.3 \%, \mathrm{P}=0.005)$. Thromboembolism was reported in $10.8 \%$ of all patients: $2.9 \%$ CYP3A4-i vs $12.6 \%$ rivaroxaban-only, $\mathrm{P}=0.0009 ; 6.4 \%$ PAI vs $11.2 \%$ rivaroxaban-only, $\mathrm{P}=0.2$. In modified Poisson models adjusted for age, sex, and Charlson score, CYP3A4-i signaled higher relative risk (RR) of bleed (RR: $1.8,95 \%$ CI: 1.1-3.0). A 10-year increase in age also was associated positively with bleeding (RR: $1.6,95 \% \mathrm{CI}$ : 1.2-2.1) but inversely with thromboembolism (RR: 0.8 , 95\% CI: 0.7-0.9).

Conclusion: In our health care system, patients on rivaroxaban plus CYP3A4-i were twice as likely to experience bleeding as patients on rivaroxaban alone. CYP3A4-i users also had less thromboembolism, suggesting pharmacologic interaction rather than chance association. While bleeding risk increases with age, increased age cannot explain lower rates of thromboembolism. Our data suggest that bleeding rates for rivaroxaban may be modulated by coadministration of agents that undergo CYP3A4 metabolism.

Keywords: cardiovascular disease, pharmacy, observational study, quality of care, ambulatory/outpatient care, pharmaceutical prescribing/use/costs

\section{OA7.02}

Impact of the Latest USPSTF Recommendations for Statin Use for Primary Cardiovascular Prevention on Payers and Care Systems

JoAnn Sperl-Hillen, ${ }^{1}$ A. Lauren Crain, ${ }^{1}$ Karen L. Margolis, ${ }^{1}$ Thomas Kottke, ${ }^{2}$ Patrick J. O'Connor, ${ }^{1}$ Susan Cooper, ${ }^{2}$ Daniel Rehrauer, ${ }^{2}$ Peter Marshall ${ }^{2}$

${ }^{1}$ HealthPartners Institute, Minneapolis, $\quad M N$; ${ }^{2}$ HealthPartners, Minneapolis, $M N$

Background: The U.S. Preventive Services Task Force (USPSTF) recently issued a primary prevention recommendation for statin use for individuals age 40-75 years with a cardiovascular $(\mathrm{CV})$ risk factor and a 10-year atherosclerotic CV disease (ASCVD) risk score $>10 \%$ (grade B rating). Because the Affordable Care Act requires insurers to cover preventive services with USPSTF ratings of A or B without cost sharing, the impact of this USPSTF recommendation is of interest to payers and care systems.

Methods: Electronic health record data were extracted at all adult primary care office encounters in a large Midwestern care system from June 1, 2016, to May 31, 2017, and processed through a clinical decision support system called CV Wizard that algorithmically identified CV risk factors, calculated a 10-year ASCVD risk score, and assessed current statin use.

Results: 207,163 unique individuals age 40-75 were identified; 5809 (7.63\%) were excluded for known CV disease; $119,786(57.8 \%)$ had at least one USPSTF CV risk factor criteria (12.2\% diabetes, $31.2 \%$ hypertension, $10.6 \%$ smokers, and $33.6 \%$ dyslipidemia); and 46,173 of 207,163 (22.3\%) met additional CV risk criteria of 10-year ASCVD risk score $>10 \%$. Only 13,594 of $46,173(22.9 \%)$ of these individuals were on a statin. A total of 49,815 (24\%) were determined to be eligible for statin therapy when including an additional $3642(1.7 \%)$ currently on a statin who would likely meet USPSTF criteria if their statin was stopped (10year ASCVD risk score 7.5\%-9.9\%). Using the average cost of $\$ 60$ annually per person (atorvastatin $\$ 5 /$ month, simvastatin \$2/month, and rosuvastatin \$11/month), the annual cost of statin medication for primary $\mathrm{CV}$ prevention for patients in this care system is approximately $\$ 3$ million (not including cost related to additional office visits, labs, and drug side effects and toxicity). 
Conclusion: One-fourth of all individuals age 40-75 in a large Midwestern care system met USPSTF criteria for statin use for primary CV prevention, and only about onefourth of them were on a statin. While statin use for primary prevention using the USPSTF criteria is considered by most to be cost-effective and of substantial net clinical benefit, many operational and cost challenges exist to providing this service to patients.

Keywords: cardiovascular disease, pharmaceutical prescribing/costs

\section{OA7.03 \\ Evolving Cardiovascular Disease Risk Factors in a Somali-American Immigrant Community}

Bjorn Westgard,,$^{1}$ Brian Martinson, ${ }^{1}$ Mike Maciosek, ${ }^{1}$ Farhiya Farah, ${ }^{1}$ Sandi Wewerka ${ }^{1}$ Douglas Pryce $^{2}$

\section{${ }^{1}$ HealthPartners, Minneapolis, MN; ${ }^{2}$ Hennepin County Medical Center, Minneapolis, MN}

Background: Since the early 1990s, Somali people from East Africa have been migrating to and assimilating in more developed countries where new environments have affected dietary intake and energy expenditures. The effects of these new health risks among Somalis warrant assessment to prioritize public health measures to prevent metabolic syndrome and cardiovascular disease (CVD). However, assessments to date have largely employed self-reported and clinic data. Our current study provides the best estimate of changing CVD risks in the Somali community.

Methods: The prevalence of CVD risk was determined in Somali adults in the Twin Cities metro area at two points in time by trained ethnicity- and gender-concordant community health workers. In 2001, a cluster-randomized sample of Somali household members $(\mathrm{N}=253)$ was surveyed. In 20152016, a larger respondent-driven sample of the growing Somali community $(\mathrm{N}=1180)$ was surveyed to approximate a random sample. In both efforts we measured body mass index, waist circumference, blood pressure, hemoglobin A1c, total cholesterol, and high-density lipoprotein as well as historical variables of smoking, health insurance, and time in the United States, among other demographic variables.

Results: In the 2001 survey, 58\% were female, with $50 \%$ age $18-34$ years, $21 \%$ age $35-49,17 \%$ age $50-64$, and $11 \%$ older than 65 . In the 2015-2016 survey, 61\% were female, with $35 \%$ age $18-34,20.5 \%$ age $35-49,20 \%$ age $50-64$, and $24.6 \%$ older 65 . Prevalence of diabetes, hypertension, and dyslipidemia has increased significantly, from $5.9 \%$ to $24 \%, 7 \%$ to $34.6 \%$, and $5 \%$ to $19 \%$, respectively. Obesity has increased from $61 \%$ to $72 \%$. Overall smoking has not changed, at $11 \%$, but has increased among young and middle-aged men. In the recent survey, $97.7 \%$ of participants have lived in the United States for over 10 years compared to $4 \%$ in 2001 , and the health insurance rate has decreased from $83 \%$ to $78 \%$.
Conclusion: Our study provides the best longitudinal profile of CVD risk in the Somali community. Rising rates of diabetes, hypertension, and dyslipidemia are of concern, while obesity and smoking are of particular concern in women and men, respectively. This research identifies the most modifiable targets of CVD risks to which treating clinicians, health systems, and public health efforts should devote their resources.

Keywords: cardiovascular disease, racial/ethnic differences, health, health care

\section{OA7.04 \\ Population Segmentation by Cardiometabolic Disease Burden and Patient's Need}

Xiaowei Yan, ${ }^{1}$ J.B. Jones, ${ }^{1}$ Josh Liberman, ${ }^{1}$ Lily (Shuting) Liang, ${ }^{1}$ Walter F. Stewart ${ }^{1}$

${ }^{1}$ Sutter Health, Walnut Creek, CA

Background: Population health management depends, largely, on segmenting patients based on common clinical needs into groups to facilitate customized services. Previous segmentation work has relied primarily on experiential and ad hoc methods (eg, disease management), not on machine learning methods (clustering) that account for how various features (ie, comorbidities and overall disease burden) are distributed in a population. However, clustering methods applied directly to electronic health record (EHR) data may not yield reproducible and distinct segments. Herein we present a segmentation using consensus clustering approach with features derived from the EHR and discuss its implications to customized care models.

Methods: We extracted a random sample of 10,000 primary care patients who were age 35 years or older from an integrated health system in Northern California and derived features from EHR data that represent disease burden cardiometabolic biomarkers A1c, low-density lipoprotein, high-density lipoprotein, triglyceride, estimated glomerular filtration rate, etc - along with patient characteristics. We applied consensus clustering method to those features, which resulted in assignment of patients to 3 to 5 clusters, along with reliability and reproducibility metrics of the clusters.

Results: The three-segment model was the most reliable solution, with consensus probability above $80 \%$ for each cluster (ie, the probability of assigning each pair of subjects to the same cluster is $80 \%$ or above). Three clusters had distinct disease profiles; $58 \%$ were assigned to segment 1 , for which mean age was 52 years, with low prevalence of diabetes (7\%), cardiovascular, and coronary heart diseases. Segment 2 accounted for $22 \%$ of the population, which had higher prevalence of cardiometabolic conditions (diabetes $17 \%$, hypertension $60 \%$, and dyslipidemia 60\%), atrial fibrillation (8.3\%), and severe chronic kidney disease $(15.4 \%$ in stage 3 or above). Segment 3 accounted for the rest, $20 \%$, with very high prevalence of cardiometabolic conditions 
(diabetes 31\%, hypertension 65\%, and dyslipidemia 79\%) and high cardiovascular disease risks as well.

Conclusion: Quantification of the underlying structure of co-occurrence, disease severity, disease control, and other relevant factors (eg, body mass index) informs how distinct pathophysiologic subtypes emerge and persist in populations. We identified three distinct patient segments; each reflects a unique set of underlying pathophysiology features, which paves the path toward segment-specific or customized care models for population health management. Keywords: cardiovascular disease, primary care, health care organization, chronic disease, epidemiology

\section{P3.01}

Racial Disparities in Hypertension: Decomposing the Effects of Risk Factor Distribution and Risk Factor Impact on Racial Disparities

Suma Vupputuri, ${ }^{1}$ Romsai Boonyasai, $^{2}$ Kevin
Rubenstein, ${ }^{1}$ Alphonse Derus ${ }^{1}$ Christine Truong ${ }^{1}$

${ }^{1}$ Kaiser Permanente Mid-Atlantic States, Rockville, MD; ${ }^{2}$ Johns Hopkins School of Medicine, Baltimore, MD

Background: Nearly half of Americans with hypertension have uncontrolled blood pressure (BP). Control rates are consistently higher in white patients than black patients. The purpose of this study is to identify covariates that contribute to racial disparities in BP control using the Oaxaca-Blinder decomposition method (OBDM).

Methods: We included white and black patients $\geq 21$ years of age with $\geq 2$ outpatient diagnoses of hypertension and $\geq 2$ BP measures. Uncontrolled BP was defined as $\geq 2$ BP measures $\geq 140 / 90 \mathrm{mmHg}$. Covariates of interest included demographics, baseline BP measures, clinical factors, utilization, provider characteristics, and engagement with health care. Distributions of covariates were calculated by race; multivariable logistic regression models were constructed overall and by race; and OBDM was used to determine the proportion of the racial disparity in uncontrolled BP that could be reduced by equalizing select characteristics as well as the proportion of the disparity due to differential impact of covariates.

Results: We identified 34,900 adult Kaiser Permanente Mid-Atlantic States patients, of whom 15,907 (46\%) had uncontrolled BP and 22,363 (64\%) were black. Uncontrolled BP occurred in $48 \%$ of blacks compared to $41 \%$ of whites. Blacks were significantly younger and more likely to have household income $<\$ 25,000$, diabetes, female gender, and higher average systolic BP. Blacks were less likely to engage with the kp.org website and have race-concordant providers. In a multivariable logistic regression model, blacks had $20 \%$ increased odds of having uncontrolled BP. The contribution of the differences in the average values of the study covariates between blacks and whites (OBDM covariate effect) accounted for $40 \%$ of the overall racial disparity.
Age and kp.org use contributed to worsening the whiteblack disparity by $22 \%$ and $8 \%$, respectively, while gender, baseline systolic BP, and diabetes contributed to diminishing the disparity by $5 \%$. The contribution of the difference in how covariates impact BP control (OBDM coefficient effect) accounted for $60 \%$ of the overall racial disparity.

Conclusion: It may not be possible to reduce the racial disparity in uncontrolled BP by equalizing the currently reported covariate values in blacks and whites (since many of these covariates are nonmodifiable). Supplementing health system data with patient-reported outcomes may be needed to effectively use OBDM and parse contributors of disparity. Keywords: cardiovascular disease, observational study, racial/ethnic differences, health care, chronic disease, epidemiology

\section{P3.02}

Central Obesity and Visceral Adipose Tissue Are Not Associated With Incident Atherosclerotic Cardiovascular Disease Events in Older Men

John Schousboe, ${ }^{1}$ Allyson Kats, ${ }^{2}$ Lisa Langsetmo, ${ }^{2}$ Tien Vo, ${ }^{2}$ Brent Taylor, ${ }^{2}$ Ann Schwartz, ${ }^{3}$ Peggy Cawthon, ${ }^{4}$ Beth Lewis, ${ }^{5}$ Elizabeth Barrett-Connor, ${ }^{6}$ Andrew Hoffman, ${ }^{7}$ Eric Orwoll, ${ }^{8}$ Kristine Ensrud ${ }^{2}$

${ }^{1}$ HealthPartners Institute, HealthPartners, Bloomington, $M N$; ${ }^{2}$ Division of Epidemiology and Community Health, University of Minnesota, Minneapolis, MN; ${ }^{3}$ Division of Biostatistics and Epidemiology, University of California, San Francisco, San Francisco, CA; ${ }^{4}$ California Pacific Medical Center Research Institute, San Francisco, CA; ${ }^{5}$ University of Alabama at Birmingham, Birmingham, $A L$; ${ }^{6}$ University of California, San Diego, San Diego, CA; ${ }^{7}$ Stanford University, Palo Alto, CA; ${ }^{8}$ Oregon Health \& Science University, Portland, OR

Background: Visceral adipose tissue (VAT) and a high proportion of central vs peripheral adipose tissue have been shown to be important predictors of incident atherosclerotic cardiovascular disease (ASCVD) events in middle-aged individuals, but this has not been established in older men. Dual-energy X-ray absorptiometry (DXA) can accurately measure VAT and other regional adipose tissue depots compared to computed tomography and magnetic resonance imaging. Our study aim was to estimate the associations of DXA-VAT and the proportion of central vs peripheral adipose tissue (android-to-gynoid fat mass ratio) with incident ASCVD (myocardial infarction, coronary heart disease death, or fatal or nonfatal stroke) among older men. Methods: 2,899 older men (mean age: 76.3 [SD: 5.5] years) were enrolled in the Outcomes of Sleep Disorders in Older Men study; they had valid baseline measures of VAT on DXA body composition studies (Hologic QDR4500A), complete covariate data, and rigorous adjudication of incident ASCVD events. Participants were surveyed by postcard every 4 
months for any potential ASCVD events, and 2 physician experts reviewed all relevant medical records to determine if an event had occurred. We used proportional hazards models to estimate the hazard ratios (HR) for incident ASCVD events per SD increase of VAT or android-to-gynoid fat mass ratio, adjusted for age, race, education, systolic blood pressure, smoking status, oxidized low-density lipoprotein level, treatment for hypertension, statin use, aspirin use, and presence of diabetes mellitus.

Results: Over a mean follow-up time period of 7.9 (SD: $3.4)$ years, 424 men (14.6\%) had an incident ASCVD event. Neither VAT nor android-to-gynoid fat mass ratio were associated with incident ASCVD events in either unadjusted or multivariable-adjusted analyses (multivariable-adjusted HR per SD increase of 1.03 [95\% CI: 0.93-1.14] and 1.06 [95\% CI: 0.96-1.17], respectively).

Conclusion: Neither VAT nor android-to-gynoid fat mass ratio are associated with ASCVD events in older men. These results are not generalizable to women or middle-aged individuals, and further research of DXA measures of VAT with ASCVD in these populations is warranted. Further research is also warranted to explain how and why the association of central obesity with incident ASCVD may change with age.

Keywords: cardiovascular disease, epidemiology

\section{P3.03}

Blood Pressure Checks and Diagnosing Hypertension (BP-CHECK): Design and Methods of a Randomized Controlled Diagnostic Study Comparing Clinic, Home, Kiosk, and 24-Hour Ambulatory BP Monitoring

Beverly Green, ${ }^{1}$ Melissa Anderson, ${ }^{1}$ Jerry Campbell, ${ }^{2}$ Andrea Cook, ${ }^{1}$ Kelly Ehrlich, ${ }^{1}$ Sarah Evers, ${ }^{1}$ Yoshio Hall, ${ }^{3}$ Clarissa Hsu, ${ }^{1}$ Dwayne Joseph, ${ }^{2}$ Pedja Klasnja, ${ }^{1}$ Karen L. Margolis, ${ }^{4}$ Sean Munson, ${ }^{3}$ Matthew Thompson ${ }^{3}$

${ }^{1}$ Kaiser Permanente Washington Health Research Institute, Seattle, WA; ${ }^{2}$ patient partner (unaffiliated); ${ }^{3}$ University of Washington, Seattle, WA; ${ }^{4}$ HealthPartners, Bloomington, $M N$

Background: The U.S. Preventive Services Task Force recommends screening adults for high blood pressure (BP). If BP is high, out-of-office BPs are recommended before making a new diagnosis of hypertension, preferably using 24-hour ambulatory BP monitoring (ABPM), because over 30\% have normal BPs outside of clinic or "white coat hypertension" with risk of cardiovascular events and death similar to those without hypertension. Currently, few physicians use and few patients have heard of ABPM, which may be less convenient than clinic, home, or kiosk-based monitoring. Objective: The BP-CHECK randomized controlled diagnostic study will compare the accuracy and acceptability of clinic, home, and kiosk-based BP monitoring compared to ABPM for diagnosing hypertension. The study aims, design, protocols, materials, and evaluation described herein are informed by patient, health care, policy, and expert stakeholders.

Methods: Adults age 18-85 years will be recruited and randomized to routine screening via clinic screening, home BP monitoring over 5 days, or kiosk-based monitoring on 3 separate days. After completion, all participants will complete ABPM. Mean BP assessed via each screening method (clinic, home, kiosk) will be compared with 24-hour APBM to assess the accuracy of each method. We also will assess the acceptability of each method from the patient's perspective and the impact of the tests longer-term. Finally, a mixed-method analysis is planned to explore physician knowledge and beliefs about BP measurement, diagnosing hypertension, and the perceived feasibility of using each of the tested BP strategies in routine clinical practice.

Results: Stakeholder committees that included patients, health care providers, hypertension, and BP measurement experts, advocacy group, and policy leaders informed the study design and protocols. Enrollment began in May 2017 with a target of randomizing 510 participants.

Conclusion: BP-CHECK will inform which hypertension diagnostic methods are most accurate, acceptable to patients, and feasible to implement in primary care. This evidence base is critical to compare alternate methods of diagnosing hypertension to ABPM

Keywords: cardiovascular disease, technology adoption and diffusion, primary care, clinical decision-making, patient experience/satisfaction

\section{P3.04}

Using Patient Demographics and Preferences to Prioritize Clinical Preventive Services for Cardiovascular Disease

Steven P. Dehmer, ${ }^{1}$ Michael V. Maciosek, ${ }^{1}$ Amy B. LaFrance, ${ }^{1}$ Thomas J. Flottemesch ${ }^{2}$

${ }^{1}$ HealthPartners Institute, Minneapolis, MN; ${ }^{2} I B M$ Watson Health, Minneapolis, $M N$

Background: When it comes to prevention, primary care providers face a wide range of competing demands with respect to following national care guidelines and recommendations, achieving quality measures, and meeting the needs of patients often presenting with more acute needs. To help shed light on this issue, the National Commission on Prevention Priorities developed a consistent methodology of population measures for comparing clinical preventive services on the basis of their relative value. Using the case of preventive services for cardiovascular disease (CVD), this study seeks to advance the relevance of these prioritysetting tools to the point of care by incorporating patient demographics and preferences.

Methods: A microsimulation model was developed to estimate patient- and population-level impacts of three services recommended by the U.S. Preventive Services Task Force: aspirin counseling for the primary prevention of CVD 
and colorectal cancer; cholesterol screening and treatment; and screening and treatment for hypertension. Analyses compare lifetime outcomes for U.S.-representative birth cohorts, stratified by sex and race/ethnicity, with and without access to each clinical preventive service. Each synthetic patient in the model serves as its own control. Primary outcomes were overall health impact (measured by the net difference in lifetime quality-adjusted life-years [QALYs]) and lifetime prevented myocardial infarctions and strokes.

Results: Across all adults, overall health impact was highest for hypertension screening and treatment (16,000 QALYs per 100,000). However, this result was driven by the large benefits for women (18,000 QALYs); whereas for men, cholesterol screening and treatment ranked highest for reducing CVD burden (19,000 QALYs). For both men and women specifically motivated to reduce their risk of a myocardial infarction, cholesterol screening was favored (1500 fewer myocardial infarctions per 100,000); whereas for stroke, hypertension screening was favored (1000 fewer strokes per 100,000). Although qualitatively similar, quantitative differences by race/ethnicity were notable. For example, screening and treating for hypertension was found to prevent almost 3 times the number of strokes for non-Hispanic black women in comparison to non-Hispanic white women.

Conclusion: These results show the value of tailoring prevention priorities to individual patient characteristics and preferences and also highlight opportunities to reduce health disparities.

Keywords: primary care, health promotion, prevention, screening, racial/ethnic differences, health care, gender/sex differences, economic studies

\section{DATA SCIENCE AND NETWORKS}

\section{OA8.01 \\ The AGING Initiative Experience: A Call for Sustained Support for Team Science Networks}

Tullika Garg, ${ }^{1}$ Kathryn Anzuoni, ${ }^{2}$ Valentina Landyn, ${ }^{2}$ Alexandra Hajduk, ${ }^{3}$ Stephen Waring, ${ }^{4}$ Leah Hanson, ${ }^{5}$ Heather Whitson ${ }^{6}$

${ }^{1}$ Geisinger Health System, Danville, PA; ${ }^{2}$ Meyers Primary Care Institute, Worcester, MA; ${ }^{3}$ Yale School of Medicine, New Haven, CT; ${ }^{4}$ Essentia Institute of Rural Health, Duluth, $M N$; ${ }^{5}$ HealthPartners Institute, Minneapolis, MN; ${ }^{6}$ Duke University, Durham, NC

Background: Team science, defined as collaborative research efforts that leverage the expertise of diverse disciplines, is recognized as a critical means to address complex health care challenges, but the practical implementation of team science can be difficult. Our objective is to describe the lessons learned from our team science experience as applied to the complex and growing challenge of multiple chronic conditions (MCC). MCC is the presence of "two or more chronic conditions that collectively have an adverse effect on health status, function, or quality of life and that require complex healthcare management, decision-making, or coordination." Due to the increasing impact on U.S. society, MCC research has been identified as a high-priority research area by multiple federal agencies. Methods: To address this need, two national research entities, the Health Care Systems Research Network (HCSRN) and the Claude D. Pepper Older Americans Independence Centers (OAIC), formed the Advancing Geriatrics Infrastructure and Network Growth (AGING) Initiative to build nationwide capacity for MCC team science. Initiative structure and barriers/solutions are described (eg, addressing cultural differences, including diverse perspectives and expertise).

Results: In its first 3 years, the AGING Initiative demonstrated success in advancing team science for MCC. Eighteen manuscripts have been published or are in press, and an additional 11 manuscripts are in development. In addition to manuscripts, several new grant applications have been produced by AGING Initiative research teams. From the AGING Pilot Projects Program, 7 grant applications have been submitted as a result of the 6 pilot projects in the first two cycles of funding.

Conclusion: Team science has emerged as an important method to bring together diverse skill sets and data to solve complex clinical problems. The AGING Initiative experience demonstrates that, in less than 3 years, building a team science platform can expand capacity and catalyze collaboration around a complex health problem. Building nationwide infrastructure to foster team science requires understanding different workplace cultures, efficiency in data sharing, thoughtfully designing organizational structure, disseminating to expand a diverse community, engaging the next generation of team scientists, and applying a flexible, iterative approach to create solutions to unanticipated barriers during implementation.

Keywords: aging, elderly, geriatrics, complex disease management, multiple chronic conditions, dissemination, implementation, innovation, communication of research findings

\section{OA8.02}

Distributed Health Data Networks: Implementing a Scalable Query Interface Within PopMedNet for Use in Large-Scale Diverse Networks

Hozefa Divan, ${ }^{1}$ Kyle Erickson, ${ }^{1}$ Kimberly Barrett, ${ }^{1}$ Zachary Winer, ${ }^{1}$ Jessica Malenfant, ${ }^{1}$ Chayim HerzigMarx, ${ }^{1}$ Jeffrey Brown ${ }^{1}$

\section{${ }^{1}$ Harvard Pilgrim Health Care Institute, Boston, MA}

Background: There is significant need to utilize electronic health data across distinct health care institutions for public health surveillance and research purposes. Distributed data 
networks (DDNs) typically use a common data model to standardize data across institutions (data partners) to facilitate rapid distributed analyses while keeping data behind local firewalls. However, data partners often have varying governance and data infrastructures that can lead to substantial ecosystem heterogeneity across partners, including use of various relational database management system (RDBMS) platforms (ie, Oracle, SQL Server, PostgreSQL). To enable rapid distributed querying across heterogeneous technical ecosystems we developed a novel, open-source tool within the PopMedNet (PMN) platform that bridges these platforms and software challenges. This tool enables investigators to compose and distribute custom queries via a user interface in PMN, referred to as the MenuDriven Query (MDQ).

Methods: The MDQ tool includes a point-and-click, scalable query interface that supports complex logic for users to define cohorts of interest (eg, medical codes, date ranges) for use in distributed queries that can be executed in various technical ecosystems. Underpinning the MDQ is a series of file conversions, e-workflow, and software-enabled governance. The tool is modularized, enabling functionality to be database-agnostic and can be run against multiple RDBMS platforms without custom programming, as it utilizes widely adopted data exchange formats.

Results: MDQ testing and validation process has been implemented and has shown consistent, valid results across database platforms; this process has informed tool development and continuous enhancements. As further realworld evidence for the tool, over 4300 unique MDQ-to-site requests have been submitted within PCORnet, a DDN, since early 2016.

Conclusion: This new tool is helping to close gaps by creating more opportunities for investigators to ask research questions more easily, flexibly, and rapidly within their DDNs while adhering to their local governance and technology policies. There is continued work to improve the MDQ tool in ways that will enable even more scalability in designing reusable query interfaces and electronic workflows.

Keywords: information technology, dissemination, implementation, innovation

OA8.03

Using Electronic Medical Records to Identify Complex Health Outcomes

Mary Anne Armstrong, ${ }^{1}$ Maqdooda Merchant, ${ }^{1}$ Amy Alabaster, ${ }^{1}$ Tina Raine-Bennett, ${ }^{1}$ Debbie Postlethwaite ${ }^{1}$

\section{${ }^{1}$ Kaiser Permanente, Oakland, CA}

Background: Electronic medical records (EMRs) have been used successfully in health research to identify outcomes and risk factors in a cohort of interest. The purpose of this study was to use EMR data to develop and validate algorithms using ICD-9-CM codes, CPT codes and natural language processing
(NLP) to identify uterine perforations and intrauterine device (IUD) expulsions following IUD insertions.

Methods: All women with an IUD insertion in a 7-year period were identified using the Kaiser Permanente Northern California virtual data warehouse (VDW). Separate algorithms were developed to identify potential uterine perforations and expulsions using data from the VDW, the surgical procedure database (OpTime), and the Clarity and radiology notes tables. Uterine perforations were identified electronically with an algorithm using a combination of codes and NLP. There were structured codes for identification of suspected or confirmed perforations but not for delineating them as complete or partial. There were no structured codes specific for IUD expulsion, so algorithms using NLP terms only were developed to identify this outcome. For both outcomes, 100 potential cases were randomly chosen for manual medical record review and validation. Positive predicted values (PPV) comparing the algorithm results to the manual medical record review results (gold standard) with 95\% confidence intervals were calculated and used to assess the accuracy of the algorithms. Results: There were 444 potential perforations identified among 168,744 IUD insertions. The PPV among 100 randomly selected potential perforation cases was $77 \%(95 \%$ CI: $68 \%-85 \%$ ), with 16 that were classified as not a case and 7 that could not be classified with any certainty. Based on medical record review, 28 of 77 were classified as partial, 48 were classified as complete, and 1 did not have enough information for classification as partial or complete. There were 4185 potential expulsions identified among 168,744 IUD insertions. The PPV among 100 randomly chosen potential expulsions was 77\% (95\% CI: 68\%-85\%), with 2 of 100 potential cases classified as undetermined.

Conclusion: The PPV was $77 \%$ for both uterine perforation and expulsions, indicating excellent performance of both algorithms for the capture of these complex health outcomes. Keywords: virtual data warehouse, natural language processing

\section{OA8.04}

Cross-Network Directory Service: A Sociotechnical Platform to Enable Meaningful Collaboration Across Organizations

\author{
Jessica Malenfant, ${ }^{1}$ Jenny Hochstadt, ${ }^{1}$ Kimberly \\ Barrett, ${ }^{1}$ Zachary Wyner, ${ }^{1}$ Daniel Dee, ${ }^{2}$ Dean \\ Corriveau, ${ }^{2}$ Bridget Nolan, ${ }^{1}$ Chayim Herzig-Marx, ${ }^{1}$ \\ Jeffrey Brown ${ }^{1}$ \\ ${ }^{1}$ Harvard Medical School and Harvard Pilgrim Health Care \\ Institute, Boston, MA; ${ }^{2}$ Avacoda LLC, Amherst, MA
}

Background: With the proliferation of health data networks, increasingly diverse data are becoming available for secondary purposes like research. Enabling access to the wealth of data resources and potential collaborators 
could help pave the way for a learning health system. We describe our implementation approaches in creating CrossNetwork Directory Service (CNDS) software. This pilot created a secure, sustainable sociotechnical platform that enables people to form new partnerships to discover and share characteristics about data sources and expertise not previously accessible to them. We demonstrate CNDS using the U.S. Food and Drug Administration's Sentinel system and Patient-Centered Outcomes Research Institute's PCORnet distributed networks.

Methods: CNDS enables users to discover data and collaborators in accordance with local governance policies. Features include 1) a flexible data model that enables quick changes to what can be collected as metadata standards and inventories change, 2) software that dynamically generates the user interface in real time, and 3) cross-network search and query capability. CNDS is a web service with a database and application programming interface server for communicating between web applications built on PopMedNet ${ }^{\mathrm{TM}}$ version 6.0. Sentinel and PCORnet user, organization, and data source information were populated by the CNDS test networks.

Results: The data model has been successfully integrated with the CNDS infrastructure. The metadata management module, search functionality, and cross-network query capability have been implemented in the CNDS test environments. We have demonstrated the ability to send a query from a CNDS Sentinel network to the PCORnet network without disrupting existing workflows while adhering to the various governance policies.

Conclusion: Challenges to be discussed include defining metadata vs data; striking a balance between developing a flexible data model and the impacts of fully implementing it as a dynamic application interface; and keeping current on emerging health IT standards and best practices. CNDS is valuable beyond its ability to search metadata and send queries across PopMedNet networks. Future work includes expanding to more people, organizations, and data sources and incorporating this proof-of-concept scaffolding into the current PopMedNet codebase.

Keywords: ownership, governance, health care organization, information technology, research administration, contracting, operations, dissemination, implementation, innovation

\section{VARIOUS/MISCELLANEOUS}

\section{OA9.01 \\ Streamlining Hepatitis C Screening to Treatment in the Mid-Atlantic Region}

Carla Rodriguez, ${ }^{1}$ Kevin Rubenstein, ${ }^{1}$ Cabell Jonas, ${ }^{1,2}$ Yan Sun, ${ }^{2}$ Michael Horberg, ${ }^{1}$ Bernadette Loftus ${ }^{2}$

${ }^{l}$ Mid-Atlantic Permanente Research Institute, Rockville, MD; Mid-Atlantic Permanente Medical Group, Rockville, MD
Background: Kaiser Permanente Mid-Atlantic States implemented a hepatitis C virus (HCV) care pathway (Pathway) to improve the identification and care of patients with HCV, particularly those born from 1945 to 1965 (birth cohort) who bear the greatest burden of disease. A screening alert for patients without a prior HCV antibody $(\mathrm{Ab})$ screen prompts clinicians to order a specific $\mathrm{HCV} \mathrm{Ab}$ that triggers reflex testing for HCV RNA, hepatitis B virus (HBV) and human immunodeficiency virus (HIV) on stored samples of those patients testing $\mathrm{HCV} \mathrm{Ab+.} \mathrm{The} \mathrm{order} \mathrm{code} \mathrm{also} \mathrm{engages}$ coordinators to assist patients and support clinical workflow. Our objective was to evaluate whether the Pathway improved the carriage of patients through each step along the HCV care continuum and increased HIV and HBV screening.

Methods: We used electronic health record data to retrospectively compare patients' successful passage from screening to $\mathrm{HCV}$ treatment during two 16-month periods before and after the Pathway began. Proportional hazards models compared the time to HCV, HIV and HBV screening, follow-up HCV communication, fibrosis staging, and treatment, adjusting for potential confounders. Chi-squared tests compared the difference in proportions receiving $\mathrm{HCV}$ RNA and genotyping. We evaluated fibrosis staging and treatment since initiation of the Pathway among patients with a Pathway order code versus usual care.

Results: Over 72,000 of 506,000 participants were HCVscreened. Comparing the Pathway to the prior era, the adjusted hazard ratio (aHR) was 2.89 (95\% CI: 2.83-2.95) for $\mathrm{HCV}$ screening, with greater improvement within the birth cohort (6.80 [6.60-7.00]). Among Ab+ patients, aHR was 2.09 (1.77-2.48) for HIV screening and 0.94 (0.82-1.08) for $\mathrm{HBV}$ screening, and $\mathrm{HCV}$ confirmatory testing increased from $85 \%$ to $93 \%(\mathrm{P}<0.001)$. Among RNA+ patients, aHR was 1.77 (1.50-2.08) for HCV communication, and genotyping increased from $82 \%$ to $87 \%(\mathrm{P}<0.05)$. RNA+ patients with a Pathway order had a higher rate of fibrosis staging and treatment compared to those in usual care (aHR: 2.65 [95\% CI: 2.1-3.32] and aHR: 1.45 [95\% CI: $1.07-$ 1.98], respectively).

Conclusion: The Pathway was associated with improved HCV screening and care, as well as improved HIV screening. Research to understand effective translation to other health systems is needed.

Keywords: observational study, chronic disease, quality improvement, epidemiology

OA9.02

Metabolic Outcomes for Type 2 Diabetes Patients on New Antidiabetic Drug Classes Compared With Those on Traditional Classes in Central Texas

\author{
Stephanie Yu, ${ }^{1}$ I-Chia Liao, ${ }^{1}$ Linda Chen, ${ }^{1}$ Paul Godley, ${ }^{1}$ \\ Delaney Ivy, ${ }^{1}$ Anthony Cryar ${ }^{1}$
}

${ }^{1}$ Baylor Scott \& White Health, Temple, TX 
Background: According to the 2017 American Association of Clinical Endocrinologists/American College of Endocrinology Comprehensive Type 2 Diabetes Management Algorithm, new antidiabetic classes (NAC) of drugs - SGLT2i, GLP1-RA, DPP4i - are preferred over more traditional antidiabetic classes (TAC) - SU, TZD, meglitinide - for dual therapy after initiation of metformin. Clinical trials suggest that NAC offer favorable effects on cardiovascular risk factors with noninferior reductions in glycated hemoglobin $(\mathrm{HbAlc})$ compared with TAC. This study evaluated the validity of this recommendation.

Methods: This retrospective analysis of medical and pharmacy claims from the period 2013-2015 evaluated patients $>18$ years old with ICD-9 diagnosis of type 2 diabetes mellitus (T2DM) and use of TAC at least 1 year prior to first claim for a NAC (index date) or matched claim for a $\mathrm{TAC}$ for the index date \pm 14 days. Differences in $\mathrm{HbAlc}$ and weight reduction were evaluated between the NAC and TAC cohorts. Pre-index HbA1c, Diabetes Comorbidity Severity Index, pre-index lag times, and post-index lag times were controlled in this analysis.

Results: Data from 126 NAC and 373 TAC patients were analyzed (52\% female; mean age: 58.71 years, SD: 11.77 , min-max: 24.00-85.00). Patients in the NAC and TAC cohorts had equivalent pre-index mean baseline HbAlc (9.1\% vs $8.9 \%, \mathrm{P}=0.194)$. Adjusting for covariates, patients who took an agent in the NAC had significantly greater average $-1.6 \mathrm{~kg}$ weight reductions compared to those in TAC $(\mathrm{P}=0.02)$. In addition, mean $\mathrm{HbAlc}$ reductions were not significant between NAC and TAC cohorts $(\mathrm{P}=0.49)$ while also adjusting for covariates.

Conclusion: This real-world study demonstrated that addition of agents in the new antidiabetic drug classes resulted in significant weight reductions compared with traditional antidiabetic drug classes while maintaining equivalent $\mathrm{HbA} 1 \mathrm{c}$ reduction.

Keywords: pharmacy, diabetes, clinical practice patterns/ guidelines, pharmaceutical prescribing/use

\section{OA9.03}

\section{Transmitting Unique Device Identifiers From Point of Use to Insurance Claims}

Jove Graham, ${ }^{1}$ Kevin Capatch, ${ }^{1}$ Bret Yarczower, ${ }^{2}$ Dan Krupka, ${ }^{3}$ Yasmin Zerhouni, ${ }^{4}$ Amanda Reich, ${ }^{4}$ Angela $\mathrm{Li}^{,}{ }^{5}$ Joel Weissman ${ }^{4}$

${ }^{1}$ Geisinger Health, Danville, PA; ${ }^{2}$ Geisinger Health Plan, Danville, PA; ${ }^{3}$ Twin Peaks Group, Lexington, MA; ${ }^{4}$ Partners HealthCare, Boston, MA; ${ }^{5}$ Blue Cross Blue Shield of Massachusetts, Boston, MA

Background: Patients, providers, insurance payers, and manufacturers need reliable information on the performance and safety of implanted medical devices such as coronary stents, but availability of those data has been lacking.
Billing records and claims employ standard codes for procedures but lack information on implanted devices. In 2013, the U.S. Food and Drug Administration began requiring manufacturers to label devices with unique device identification (UDI) numbers, but hospitals and payers must decide how to leverage that information in their own information systems. The purpose of this project was to demonstrate, with two different provider/payer sites, the feasibility of transmitting UDIs to insurance claims.

Methods: Implementation teams at two provider institutions (Geisinger Health and Partners HealthCare), working with two insurance providers (Geisinger Health Plan and Blue Cross Blue Shield of Massachusetts, respectively) designed different strategies for capturing point-of-use information on devices implanted in the cardiac catheterization laboratory and transmitting that information to the insurance claim. After the design phase, strategies were implemented at each system through collaboration with internal information technology and external vendor teams.

Results: The first insurance claims containing UDIs for implanted cardiac devices were processed at both sites in October 2017. At both institutions, barcode labels were scanned at point-of-use to establish links between patient and device. At Geisinger, new records containing UDIs were created by inventory management software and sent to billing, then mapped to the note field of the electronic claims transaction. At Partners, the UDI was transmitted to billing via the electronic health record and an extension rule within the record was created to write this information to the claim if specific criteria were met.

Conclusion: Integrating device-specific information into claims will be an important step in allowing researchers who use health plan data to evaluate devices in the same way that we study surgical procedures or prescription drug use. This implementation project and the lessons learned along the way demonstrate that there are multiple feasible pathways to include UDI numbers in claims in order to provide better real-world data to evaluate long-term outcomes associated with specific products.

Keywords: information technology, health care, financing, insurance, premiums, costs, resource use, patient safety

\section{OA9.04}

\section{A Novel Mixed-Methods Approach to Identifying Contextual Patterns in Treatment Decision-Making: The Ethnoarray}

\section{Katharine Rendle, ${ }^{1}$ Sarah Garrett, ${ }^{2}$ Corey Abramson, ${ }^{3}$ Daniel Dohan²}

${ }^{1}$ University of Pennsylvania, Philadelphia, PA; ${ }^{2}$ University of California, San Francisco, San Francisco, CA; ${ }^{3}$ University of Arizona, Tucson, $A Z$

Background: In patient-centered outcomes and health services research, qualitative methodologies have been 
embraced with enthusiasm for their ability to capture individual and contextual factors that shape care experiences and medical decision-making. From interviews to ethnographic observation, these approaches provide insight into the complex ways factors interact to shape outcomes that are difficult to capture using quantitative approaches alone. However, communicating and identifying patterns in qualitative data remains challenging, particularly in large data sets that feature complex multilevel data. Drawing from two longitudinal qualitative studies examining treatment decision-making in breast cancer patients $(\mathrm{N}=36)$ and late-stage melanoma patients $(\mathrm{N}=13)$, we present a novel approach modeled after the genetic microarray to analyze and display complex qualitative data.

Methods: We used an iterative mixed-methods approach that combines thematic qualitative analysis with hierarchical clustering to produce a patterned heatmap ("ethnoarray"). For each dataset, we completed the following four-stage analysis: First, using the constant comparative method, we inductively and deductively coded interview and ethnographic data. Second, based on thematic coding and literature review, we identified influential factors relevant to treatment decisionmaking. Third, using Atlas.ti, we created a code co-occurrence table to identify presence or absence of each decision-making factor. Fourth, using R, we applied complete-link clustering to sort patients (columns) and decision-making factors (rows) by statistical proximity, resulting in a visual heatmap that identifies patterns across patients and factors. Last, we created narrative summaries for each clustered group based on qualitative and clinical data.

Results: We will present the two resulting ethnoarrays, highlighting how the tool can be used to identify similarities and differences across and within patients. We will discuss how this tool can be applied to other types of qualitative data, and how it can help to advance rigor and transparency in qualitative and mixed-methods research.

Conclusion: Although treatment decision-making is a wellstudied topic, identifying patterns across patients without losing the context captured by qualitative methods remains challenging. We present a novel analytic tool that can represent and retain these contextual complexities and can support rigorous and transparent qualitative health research in embedded research environments.

Keywords: cancer, sociology, ethnography, qualitative research, clinical decision-making

\section{AGING AND MULTIMORBIDITY}

\section{P1.01 \\ Care Transition Intervention and Hospital Readmission: Experience From Baylor Scott \& White Health}

\author{
Rashmita Basu, ${ }^{1}$ Alan Stevens ${ }^{1}$
}

${ }^{1}$ Baylor Scott \& White Health, Temple, TX

Background: In response to the Centers for Medicare and Medicaid Services (CMS) hospital readmission penalty policy, many hospitals across the country have implemented models to keep their readmission rates low. The Care Transitions Intervention $^{\circledR}$ (CTI), a patient-centered 30-day intervention model ( 1 home visit and 3 phone calls) that aims to empower patients, was implemented at Baylor Scott \& White Health hospital during early 2011 to March 2013. The goal of this study was to examine the impact of the CTI on 30-day hospital readmission and factors that predict patient's decision not to accept the CTI after being enrolled in the program.

Methods: This retrospective analysis covered the 6 months leading to the "index" hospital admission to the 6-month period after discharge during the CTI period.

Results: Primary outcome measure: 30-day hospital readmission from index hospital admission. We calculated 30day hospital readmission by constructing four groups: 1) those who enrolled and completed the full CTI intervention; 2) those who enrolled and completed only home visits, not phone calls; 3 ) those who enrolled but decided not to receive treatment (neither home visits nor phone calls); and 4) those who were eligible but declined to participate in the program. All-cause readmission rates were calculated based on CMS's definition by dividing the number of at least one subsequent hospital admission within 30 days from the index hospitalization (numerator) by total number of hospital admissions after discharge from the index hospitalization during the CTI period (denominator). Those who enrolled and completed the full CTI intervention had the lowest 30 -day readmission rate of $22 \%$. We also found that those who enrolled but did not receive any treatment had higher readmission rates $(28 \%)$ compared to those who did not enroll to the program $(25 \%)$.

Conclusion: We did not find statistically significant differences in readmission rates among groups. Results from predicting decision not to receive CTI indicated that patients with the higher number of CMS hierarchical condition categories were less likely $(\mathrm{P}=0.02)$ to receive the CTI even after they were enrolled into the program during their hospital stay. This indicates that patient comorbidity burden is an important factor to consider while offering this type of health intervention.

Keywords: aging, elderly, geriatrics

P1.02

Multimorbidity Burden and Outcomes in Adults With Heart Failure

Mayra Tisminetzky, ${ }^{1,2}$ Jerry Gurwitz, ${ }^{1,2}$ Robert Goldberg, ${ }^{2}$ Grace Tabada, ${ }^{3}$ Sue Hee Sung, ${ }^{3}$ Alan $\mathrm{Go}^{3}$

${ }^{1}$ Meyers Primary Care Institute Worcester, MA; ${ }^{2}$ University of Massachusetts Medical School, Worcester, MA; ${ }^{3}$ Kaiser Permanente Northern California Division of Research, Oakland, $C A$ 
Background: We aimed to assess multimorbidity burden and its association with clinical outcomes in a communitybased cohort of patients with heart failure (HF) according to sex, age, and HF type.

Methods: A total of 114,553 HF patients were characterized with respect to the presence of 24 chronic conditions and the overall burden of multimorbidity. Clinical outcomes included all-cause mortality and any-cause and HF-specific hospitalization. Cox proportional hazards regression models were used to account for potential confounders.

Results: Adjusted hazard ratios for all-cause mortality among those with $5-6,7-8$, or 9 or more morbidities (vs 0-4) were 1.27 (95\% CI: 1.24-1.31), 1.52 (95\% CI: 1.48 1.57), and 1.92 (95\% CI: 1.86-1.99), respectively. Adjusted hazard ratios for any-cause hospitalization among those with $5-6,7-8$, or 9 or more morbidities (vs $0-4)$ were $1.28(95 \%$ CI: $1.25-1.30), 1.47$ (95\% CI: 1.44-1.50), and $1.77(95 \%$ CI: 1.73-1.82), respectively. Adjusted hazard ratios for HFspecific hospitalization among those with 5-6, 7-8, or 9 or more morbidities (vs 0-4) were 1.22 (95\% CI: $1.19-1.26$ ), 1.39 (95\% CI: $1.34-1.44)$, and 1.68 (95\% CI: 1.61-1.74), respectively. For each outcome of interest, similar trends were found among men and women, across age groups, and by HF type. Associations between multimorbidity burden and all of the examined outcomes were especially prominent among those $<65$ years old.

Conclusion: The impact of multimorbidity on outcomes among adults with HF is substantial and may be an important consideration in clinical management and decision-making in these high-risk patients.

Keywords: cardiovascular disease, aging, elderly, geriatrics, chronic disease, epidemiology

\section{P1.03}

\section{The Association of Food Insecurity With Self- Reported Falls Among the Medicare Population}

\author{
David Mosen, ${ }^{1}$ Matthew Banegas, ${ }^{1}$ Nicole Friedman, ${ }^{2}$ \\ Elizabeth Shuster ${ }^{2}$
}

\section{${ }^{I}$ Kaiser Permanente Center for Health Research, Portland,} OR; ${ }^{2}$ Kaiser Permanente Northwest, Portland, OR

Background: Falls among the Medicare population are associated with higher morbidity and mortality. Food insecurity may contribute to increased risk of falls, although this is not well studied. The objective of this analysis was to examine the independent association of food insecurity with falls among the Medicare population.

Methods: We employed a cross-sectional survey design to analyze 25,196 Medicare Total Health Assessment (MTHA) surveys collected between November 1, 2012, and October 31, 2016. The MTHA is an operations-based survey that measures quality of care among Medicare members receiving care at Kaiser Permanente Northwest (KPNW), an integrated health care system in the Pacific Northwest. The primary outcome measure in the analysis is self-reported falls, assessed by asking whether the patient fell in the previous 12 months (yes vs no). The primary independent variable is food insecurity, assessed by asking respondents to respond to the statement, "I don't always have the money I need to buy food" (yes vs no). We used the KPNW electronic health record to procure information in three categories: demographic, which included age (continuous), sex (female vs male), and low socioeconomic status (yes vs no); clinical, comprising the Charlson comorbidity index (CCI) score $(0,1,2+)$; and utilization, which covered primary care visits $(0,1,2+)$, emergency department visits $(0,1+)$, and hospital admissions $(0,1+)$. Clinical and utilization measures were based on the 12 months prior to the MTHA survey date. Multivariable logistic regression was used to assess the association of food insecurity with self-reported falls, adjusting for demographics, clinical, and utilization measures.

Results: Approximately $2.41 \% \quad(\mathrm{n}=607)$ of survey respondents reported food insecurity, while $2.40 \%(\mathrm{n}=604)$ reported having a fall. We found that food insecurity was associated with a higher likelihood of falls (odds ratio: 1.66, 95\% CI: 1.40-1.96), adjusting for age, sex, socioeconomic status, CCI score, and utilization measures.

Conclusion: Our analysis underscores the importance of assessing food insecurity among the Medicare population, finding a significant increase in the likelihood of self-reported falls among those who do not always have the money they need to purchase food. Further research is warranted to determine whether programs to reduce food insecurity lead to reduced falls among Medicare patients.

Keywords: aging, elderly, geriatrics, social determinants of health, Medicare

\section{P1.04 \\ Racial/Ethnic Comparisons in Multiple Chronic Conditions and Health Care Utilizations Among Male Oldest-Old Patients With Cardiovascular Disease and/or Diabetes Mellitus}

\section{Jinmyoung Cho ${ }^{1}$ Alan Stevens ${ }^{1}$}

\section{${ }^{1}$ Baylor Scott \& White Health, Temple, TX}

Background: With a growing aging population, racial and ethnic minority populations have increased. Nevertheless, a great deal of uncertainty still exists regarding variability in health care services utilization, comorbidity, mortality, and longevity in minority populations. This study aims to compare multiple chronic conditions (MCC) and frequency of hospital visits between white/Caucasian and nonwhite/ non-Caucasian oldest-old male patients with diabetes mellitus or cardiovascular disease, the most common prevalent conditions among older adults.

Methods: Data from Baylor Scott \& White Health identified 5847 male patients, age 85 years or more, with cardiovascular 
disease and/or diabetes during 2010-2013. Clinical comorbidity for MCC is defined by the Charlson comorbidity index or Elixhauser comorbidity score after eliminating overlapping diagnoses. Cardiovascular disease is defined by diagnoses including hypertension, hyperlipidemia, coronary heart disease, atrial fibrillation, heart failure, peripheral arterial disease, and other atherosclerotic diseases. Bivariate correlation analyses were conducted to compare chronic conditions and number of hospital visits between whites and nonwhites. Multivariate analysis (eg, generalized linear models) examined the role that race/ethnicity has on the relationship between health care utilization and the outcome of survival.

Results: Significant differences were observed in several variables. Compared to nonwhites, whites showed a higher prevalence in all types of cardiovascular disease diagnoses (white: $10 \%-84 \%$ vs nonwhite: $6 \%-74 \%$ ); whereas whites were less frequently diagnosed with diabetes (whites: $29.5 \%$ vs nonwhites: $31.6 \%$ ). Regarding the number of hospital visits, more white male patients had emergency room visits and hospital admissions than nonwhite male patients. Furthermore, male oldest-old whites reported a higher average number of clinic visits compared to nonwhites $(\mathrm{t}=8.16, \mathrm{P}<0.001)$.

Conclusion: Bivariate correlation analyses indicate that there is a significant difference in MCC and health care utilization between white and nonwhite oldest-old male patients. A closer investigation with advanced statistical analysis is needed to assess the association between health status and health care services utilization among diverse groups (ie, white/Caucasian, black/African American, Hispanic, other) in advanced age.

Keywords: cardiovascular disease, aging, elderly, geriatrics, demographics, diabetes

\section{P1.05}

\section{Using Self-Reported Data to Segment Populations With Complex Care Needs}

\section{Elizabeth Bayliss, ${ }^{1}$ Jennifer Ellis, ${ }^{1}$ Chan Zeng ${ }^{1}$ \\ ${ }^{I}$ Institute for Health Research, Kaiser Permanente Colorado, Denver, $\mathrm{CO}$}

Background: Populations with complex care needs are not well served by a one-size-fits-all approach. Delivering tailored care management requires effective methods to segment heterogeneous populations; standard clinical data are not sufficiently informative or nuanced to serve this purpose. The Medicare health risk assessment (HRA) provides an opportunity to use patient-reported information to segment complex-needs populations for more optimal care management.

Methods: The cohort included Kaiser Permanente Colorado members age $\geq 65$ years who were previously identified as at risk for high utilization due to advanced illness and geriatric issues and who had completed an HRA between April
2014 and May 2017. HRA variables reflected self-reported quality of life, mood, activities of daily living (ADL), urinary incontinence, falls, living situation, isolation, financial constraints, nutrition, and advance directives as potentially likely to reflect different clinical and/or psychosocial profiles. We iteratively applied clustering methods to refine variable selection for cluster inputs and identify groups of individuals with distinct, actionable clinical or psychosocial profiles amenable to tailored care management. Groups were described in terms of HRA items and 1-year hospital utilization.

Results: From 9617 individuals, we identified 15 clusters that could be described based on care needs. For example, two groups ( $\mathrm{n}=273$ and $\mathrm{n}=269)$ consisted primarily of frail individuals with some memory impairment less likely to live independently: One group had poor physical and mental well-being and ADL limitations, and one had ADL limitations but good mental and physical well-being. Another group $(\mathrm{n}=287)$ was characterized by poor physical and mental well-being, pain, ADL limitations, and financial constraints. Three groups $(\mathrm{n}=1872, \mathrm{n}=1098$, and $\mathrm{n}=491)$ had few health or other limitations and relatively low utilization and were differentiated by age, presence or absence of a documented advance directive, and tobacco use.

Conclusion: Segmenting populations with chronic illness and psychosocial needs into meaningful subgroups can optimize limited care management resources and minimize patient burdens from redundant care management. Using self-reported data, we identified over 3000 individuals initially categorized as having chronic care needs requiring limited intervention and multiple smaller groups that could potentially benefit from intensive care management. Novel methods coupled with new sources of data can make care more efficient and patient-centered.

Keywords: aging, elderly, geriatrics, complex disease management, multiple chronic conditions, patient-reported outcomes and functional status

P1.06

Association Between Cancer Treatment and Mortality in Older Adults With Superficial Bladder Cancer and Multiple Chronic Conditions

Tullika Garg, ${ }^{1}$ Amanda Young, ${ }^{1}$ Maureen O'KeeffeRosetti, ${ }^{2}$ Carmit McMullen, ${ }^{2}$ Matthew Nielsen, ${ }^{3} \mathrm{H}$. Lester Kirchner, ${ }^{1}$ Terrence Murphy ${ }^{4}$

${ }^{1}$ Geisinger Health System, Danville, PA; ${ }^{2}$ Kaiser Permanente Northwest, Portland, OR; ${ }^{3}$ University of North Carolina at Chapel Hill, Chapel Hill, NC; ${ }^{4}$ Yale School of Medicine, New Haven, $C T$

Background: Bladder cancer has the highest median age at diagnosis ( 73 years), and $75 \%$ of tumors are superficial at diagnosis. Rates of progression and death are low for superficial bladder cancer (SBC). As smoking is the most common risk factor, SBC patients have high chronic 
condition burdens, which may influence cancer treatment decisions. The objective was to evaluate the association between cancer treatment and mortality in older adults with SBC and multiple chronic conditions (MCC).

Methods: Using data from 1800 older (60+ years) SBC patients (stage $\leq 1$ ) from Geisinger Health and Kaiser Permanente Northwest, we examined the association between treatment within 6 months of diagnosis (eg, bladder instillation or transurethral resection of bladder tumor) and 10-year mortality. Univariate comparisons and KaplanMeier curves were generated. Multivariable Cox models were adjusted for age, sex, race, center, SBC stage/grade, and MCC. MCC was defined as 2 baseline chronic conditions. A propensity-score analysis to estimate the causal effect of cancer treatment was performed with standardized inverse probability of treatment weights to balance the sample.

Results: Over a median follow-up of 6.6 years, 725 subjects died $(40 \%)$. Subjects who died were older at diagnosis (77 years vs 71 years) and more likely to be male $(81.7 \%$ vs $76.8 \%$ ), have normal body mass index (73\% vs $63 \%)$, be current/former smoker (76.6\% vs $71.4 \%)$, and have baseline MCC (73.2\% vs $62.6 \%)$, and were less likely to have cancer treatment (76.3\% vs $86.7 \%)$; $\mathrm{P}<0.01$ for all comparisons. Median survival was not significantly longer for treated subjects ( 8.2 years vs 6.8 years, $\log$ rank $\mathrm{P}=0.12$ ). In multivariable analyses, cancer treatment was associated with a 30\% reduction in hazard of death (adjusted hazard ratio [HR]: 0.70, 95\% CI: 0.58-0.85, $\mathrm{P}<0.0002$ ) and $\mathrm{MCC}$ was associated with a $72 \%$ increase in hazard of death (adjusted HR: 1.72, 95\% CI: 1.44-2.05, $\mathrm{P}<0.0001$ ). In the weighted analysis, cancer treatment was associated with hazard of death (HR: $0.77,95 \% \mathrm{CI}$ : $0.61-0.98, \mathrm{P}=0.0338$ ).

Conclusion: In this cohort study of older adults with SBC from two health systems, although cancer treatment was associated with a reduced hazard of death, the increased hazard due to MCC was much greater. This information may guide patient-centered decision-making in older, medically complex patients with SBC.

Keywords: aging, elderly, geriatrics, cancer, complex disease management, multiple chronic conditions

\section{P1.07 \\ Development of a Patient-Reported Outcome Measure for the Elderly Orthopaedic Hip Fracture Patient}

Naomi Johnson, ${ }^{1}$ Jennifer Renner, ${ }^{2}$ Jennah Durham, ${ }^{1}$ Lisa Schroder, ${ }^{1}$ Jeanette Y. Ziegenfuss, ${ }^{2}$ Julie Switzer ${ }^{1}$

${ }^{1}$ Department of Orthopaedics, Regions Hospital, Saint Paul, MN; ${ }^{2}$ Survey Research Center, HealthPartners Institute, Bloomington, $M N$

Background: The population of fracture patients is aging. While there are validated functional outcome measures for orthopaedic fracture patients in general, few measures address the unique concerns and needs of the oldest fracture patients. In light of this, we have developed a patientreported outcome (PRO) measure for elderly hip fracture patients. We aimed to develop a PRO measure tailored to hip fracture patients age 65 years and older for immediate practical use and as a foundation for development of future elderly outcome measures.

Methods: Preliminary open-ended telephone interviews were conducted with a group of hip fracture patients $>65$ years old or their proxies to identify aspects and outcomes of post-hip fracture care that are important to them. Based on preliminary qualitative interview responses and expert opinion, a telephone survey was created to determine the relative importance of the identified post-hip fracture care components and outcomes. These interviews were conducted with a second group of elderly hip fracture patients. Respondents were asked to rate and rank items based on importance. Survey responses will be used to create a PRO measurement tool that can be pilot tested as a basis for further studies.

Results: Of 96 subjects, 46 responded to the interview request for a final response rate of $48 \%$. Respondents first rated and then ranked items based on importance. Overall, items relating to "Providers and Care Team" were ranked as most important to $52 \%$ of respondents, followed by items related to "Communication" (24\%). "Having confidence that $\mathrm{I} / \mathrm{my}$ loved one received the best care possible" and "Having access to the surgeon" were perceived as very important for most respondents ( $98 \%$ and $76 \%$, respectively). Physical outcomes were only ranked as most important by 4 respondents ( $9 \%)$.

Conclusion: There is a need to understand factors that are of greatest importance to elderly hip fracture patients. Our survey results provide rudimentary insight into the relative importance of various outcome measures to elderly hip fracture patients. These findings will allow us to create a geriatric-specific PRO measure. Further research will include more rigorous cognitive interview and validity testing to create a PRO measure acceptable for use at a national scale.

Keywords: aging, elderly, geriatrics, quality of life, survey research and methods, quality of care, acute inpatient care, patient-provider communication, patient-reported outcomes and functional status, patient experience/satisfaction

P1.08

National Trends in Obesity in Nursing Homes, 2005-2015

Ning Zhang, ${ }^{1}$ Terry Field, ${ }^{1}$ Yanhua Zhou, ${ }^{1}$ Kathleen M. Mazor, ${ }^{1}$ Jerry Gurwitz ${ }^{1}$

${ }^{1}$ Meyers Primary Care Institute, Worcester MA

Background: The prevalence of obesity among nursing home residents increased rapidly, from $15 \%$ in 1992 to $26 \%$ 
in 2009. However, reports of the most contemporary trend of obesity prevalence in nursing homes are lacking. In this study, we will examine obesity prevalence among the longstay residents in nursing homes.

Methods: We obtained national Minimum Data Set 20052015 for all long-term residents admitted to Medicare- and Medicaid-certified facilities for at least 100 days. Residents were underweight (body mass index $[\mathrm{BMI}]<18.5$ ), healthy to overweight $(18.5 \leq \mathrm{BMI}<30)$, or obese $(\mathrm{BMI} \geq 30)$. Obese residents were further categorized as having class I $(30 \leq \mathrm{BMI}<35)$, class II $(35 \leq \mathrm{BMI}<40)$, or class III $(\mathrm{BMI} \geq 40)$ obesity. In our study, the annual prevalence of obesity was the ratio of the total number of obese long-stay residents divided by the total number of long-stay residents in a year. The prevalence of other weight categories had the similar definition. Logistic regression models were used to assess the associations of age group, race/Hispanic origin, and gender.

Results: Between 2005 and 2015, there were about 1.7 million nursing home residents in our study. The prevalence of obesity increased from $22 \%$ in 2005 to $29 \%$ in 2015 (absolute change: $7 \%$; relative change: $32 \%$ ). Class III obesity increased from 4\% in 2005 to almost $7 \%$ in 2005 (absolute change: 3\%; relative change: 75\%). In 2015, the most recent available year, women had higher obesity prevalence than men $(31 \%$ vs $25 \%, \mathrm{P}<0.001)$. Obese residents tended to be younger, had higher percentage of diabetes, heart failure, chronic obstructive pulmonary disease, arthritis, depression, and renal failure, and had lower percentage of stroke, Alzheimer's, dementia, vision impairment, and incontinence than residents who were healthy weight or overweight. Logistic regression (adjusted for age groups, race/ethnicity, and gender for obesity) showed that age was an important predictor.

Conclusion: In this national study of nursing home residents, the age-adjusted prevalence of obesity in 2005 was $28 \%$ among men and $35 \%$ among women. The corresponding values for class III obesity were $8 \%$ for men and $9 \%$ for women. Studies are needed to determine the reasons for these differences and trends.

Keywords: aging, elderly, geriatrics, long-term care

\section{P1.09}

Factors Affecting Time Between Symptom Onset and Emergency Department Arrival in Stroke Patients

Scott Le, ${ }^{1}$ Laurel Copeland, ${ }^{1}$ John Zeber, ${ }^{1}$ Jared Benge, ${ }^{1}$ Leigh Allen, ${ }^{1}$ Jinmyoung Cho, ${ }^{1}$ I-Chia Liao, ${ }^{1}$ Jennifer Rasmussen ${ }^{1}$

${ }^{1}$ Baylor Scott \& White Health, Temple, TX

Background: Despite effective treatment options, delays in presentation for acute stroke are common. Delays of more than 4 hours preclude use of tissue plasminogen activator (tPA), consistent with American Stroke Association guidelines. Furthermore, there is strong evidence that patients who are treated more quickly will have greater benefit. The purpose of this study is to assess factors associated with time between symptom onset and emergency department (ED) arrival in stroke patients.

Methods: This study included patients presenting to the Baylor Scott \& White ED in Temple, Texas, within 7 days of symptom onset between January 2015 and July 2017 and who were diagnosed with transient ischemic attack (TIA), ischemic stroke, cerebral hemorrhage, or subarachnoid hemorrhage. Data were extracted from Get With the Guidelines, a national dataset summarizing stroke cases. The dependent variable was time between discovery of stroke symptoms and presentation at ED. Covariates included patient and system measures. Factors with a bivariate association to time to ED were entered into multivariable regression models predicting time to ED: 0-4 hours vs longer (dichotomy), or vs $>4$ to 8 hours, $>8$ to 24 hours, $>24$ to 48 hours, $2-7$ days (5 groups).

Results: The cohort of 1874 patients averaged 67.5 years (SD: 14.8 ), was $50 \%$ male, $82 \%$ white and $16 \%$ black. Average time from symptom discovery to presentation was 15.0 hours (SD: 23.2); 44\% presented within 4 hours, $13 \%$ within $>4-8,25 \%$ within $>8-24,10 \%$ within $1-2$ days, and $8 \%$ within 2-7 days. Adjusted models suggested that female gender, diabetes, race, Medicaid, smoking, and substance use disorders were significantly associated with $>4$-hour presentation, while symptom onset during waking hours (7 am-9 pm; odds ratio: 8.8), higher National Institutes of Health Stroke Scale score (odds ratio: 1.04), and advance notification of ED team by emergency medical services were protective (odds ratio: 2.0). Additional factors were identified in the ordered logit analysis of time categories, including obesity.

Conclusion: A combination of timing of symptom discovery and demographic and health care factors predicts delays to presenting for stroke treatment. Such information can be incorporated into clinical policies and treatment decisions to enable personalized care and improved outcomes for patients presenting with stroke symptoms. Further research should validate predictive models and integrate research results into system processes and clinical practice to reduce time from stroke onset to presentation.

Keywords: rural health, cardiovascular disease, aging, elderly, geriatrics, quality of care, demographics, acute inpatient care, ambulatory/outpatient care, social determinants of health, gender/sex differences, diabetes, Medicaid/SCHIP, substance abuse and addiction, Medicare, complex disease management, multiple chronic conditions, health care costs, resource use, hospital, biostatistics, access to services, quality improvement, epidemiology 


\section{CANCER}

P2.01

Use of Clinical Data in Assessing Long Risk of Keratinocyte Carcinoma Among Patients With Parkinson's Disease

Sharon Lerman, ${ }^{1}$ Boaz Amichai,, ${ }^{2}$ Gabriel Weinstein, ${ }^{3}$ Varda Shalev, ${ }^{3}$ Gabriel Chodick ${ }^{3}$

${ }^{1}$ Hadassah Medical School, Hebrew University, Jerusalem, Israel; ${ }^{2} T e l$ Aviv University, Tel Aviv, Israel; ${ }^{3}$ Maccabi Healthcare Services, Tel Aviv, Israel

Background: The association between Parkinson's disease (PD) and melanoma is well recognized, but its relationship with nonmelanoma skin cancers (NMSCs) has not been studied in depth. Study objective was to assess the relationship between PD and risk of melanoma or keratinocyte carcinoma (squamous cell carcinoma [SCC], basal cell carcinoma [BCC]).

Methods: This historical cohort study used the data of 1.2 million adult members of a large health organization between 2000 and 2015. Individuals who were diagnosed with PD any time between 2000 and 2010 were retrospectively followed until August 2016 for incidence of SCC, BCC, or melanoma identified from physician diagnoses and pathology reports.

Results: The PD cohort included 7727 patients (mean age: 69.9 years, SD: 14.8 years) among the 1,251,695 study population. During follow-up, a total of 4553, 32,069, and 4015 cases of BCC, SCC, and melanoma were identified in the study population, respectively. Multivariable models revealed that older age, male sex, never smoking, residence in southern Israel, and actinic keratosis were associated with an increased risk of both BCC and SCC. PD patients age 45-64 years and 65-84 years at baseline had odds ratios of 2.11 (95\% CI: 1.40-3.18) and 1.52 (95\% CI: 1.21-1.91) for $\mathrm{BCC}$, respectively. Weak or no associations were calculated for melanoma or SCC.

Conclusion: We report a positive relationship between PD and risk of BCC. These results should stimulate greater awareness on the part of health care providers to the increased risk of BCC in PD patients.

Keywords: cancer

\section{P2.02}

\section{Defining Priorities to Improve Patient Experience in Superficial Bladder Cancer}

Tullika Garg, ${ }^{1}$ Jill Connors, ${ }^{2}$ llene Ladd, ${ }^{1}$ Tyler Bogaczyk, ${ }^{3}$ Sharon Larson ${ }^{1}$

${ }^{1}$ Geisinger Health System, Danville, PA; ${ }^{2}$ Indiana University School of Medicine, Indianapolis, IN; ${ }^{3}$ Drexel College of Medicine, Philadelphia, PA
Background: Approximately $75 \%$ of bladder cancers are superficial at diagnosis. Though muscle-invasive disease affects far fewer, much research focuses on experiences related to radical cystectomy and urinary diversion. Significant gaps remain in our understanding of the experiences and needs of superficial bladder cancer patients across the cancer continuum. The objective of this study was to understand patient experience, define care priorities, and identify targets for care improvement in superficial bladder cancer.

Methods: Patients treated for superficial bladder cancer (stage $<\mathrm{T} 2$ ) were invited to share their care experiences throughout the cancer continuum, including diagnosis, treatment, and survivorship, in focus groups. Transcripts were analyzed using conventional content analysis to identify themes and subthemes.

Results: A total of 20 patients participated in three focus groups. Mean age was 71 years, 16 participants were male, and all were white. Five primary themes emerged: access to care, provider characteristics and communication, quality of life, goals of care/influences on decision-making, and role of social support. Patients with superficial bladder cancer desired timely access to care and honest and caring provider communication. They described urinary function and emotional quality of life changes resulting from diagnosis and treatment. Avoiding cystectomy and being alive for family were the major decision influencers. In the poster, we will present themes, subthemes, and representative quotations.

Conclusion: In this qualitative study, we identified access to care, provider characteristics and communication, quality of life, values/influences on decision-making, and social support as priority areas to improve patient experience in superficial bladder cancer. Care redesign efforts should focus on improving access, enhancing provider communication, reducing side effects, and supporting caregiver roles.

Keywords: cancer, qualitative research, patient experience/ satisfaction

P2.03

Detecting Cases of Monoclonal Gammopathy of Undetermined Significance in Electronic Health Data

Mara Epstein, ${ }^{1}$ Cassandra Saphirak, ${ }^{2}$ Yanhua Zhou, ${ }^{2}$ Brenda Birmann, ${ }^{3}$ Candace LeBlanc, ${ }^{4}$ Alan Rosmarin, ${ }^{5}$ Jerry Gurwitz

${ }^{1}$ Meyers Primary Care Institute / University of Massachusetts Medical School, Worcester, MA; ${ }^{2}$ Meyers Primary Care Institute, Worcester, MA; ${ }^{3}$ Channing Division of Network Medicine, Brigham and Women's Hospital / Harvard Medical School, Boston, MA; ${ }^{4}$ Reliant Medical Group, Worcester, MA; ${ }^{5}$ UpToDate, Waltham, MA

Background: Multiple myeloma is preceded by the largely asymptomatic and incidentally diagnosed monoclonal gammopathy of undetermined significance (MGUS). 
Retrospective studies estimate that $3 \%$ of U.S. adults age $\geq 50$ years have this premalignant condition, yet MGUS patients progress to multiple myeloma at a rate of only $1 \%$ per year. The resulting uncertainty leads to heightened patient anxiety and repeated clinical surveillance. To facilitate population-based MGUS research, we are developing an algorithm to efficiently identify MGUS patients in automated health care data.

Methods: Eligible men and women were age $\geq 50$ years, sought care at a large, community-based health care provider group in central Massachusetts at least once between 2007 and 2015, and were enrolled for $\geq 12$ months. Patients diagnosed with multiple myeloma prior to or within 3 months of MGUS diagnosis were excluded. The first step of the algorithm identified eligible patients with two or more MGUS diagnosis codes in their electronic medical record (EMR) entered on different dates within a 12-month period. Next, we selected those patients who, within 90 days of MGUS diagnosis, had: A) one or more serum protein electrophoresis tests; B) a serum or urine immunofixation test; and C) an office visit with an oncologist. A targeted manual EMR review conducted by 3 nurse abstractors and adjudicated by 2 clinicians constituted the gold standard for positive predictive value calculations.

Results: The algorithm identified 833 subjects who met the first algorithm step; 429 (52\%) patients met all 4 algorithm criteria. EMRs for 250 patients in the latter group (58\%) were reviewed. Potential MGUS cases meeting all four algorithm criteria were $85 \%$ white and $51 \%$ female, had a median of 10 MGUS diagnosis codes in their EMR, and had a mean age of 74 years at diagnosis. Review of the first $20 \%$ of charts suggests a preliminary positive predictive value of $78 \%$ or greater for the 4-step algorithm; chart review is ongoing.

Conclusion: Early results from this single-site study suggest that MGUS cases can be identified with reasonable accuracy in electronic health data through a 4-step algorithm. Following completion of chart review, the algorithm will be applied to a second Cancer Research Network health care system to evaluate performance in an independent population.

Keywords: cancer, epidemiology

\section{P2.04}

\section{Adherence to Surveillance Guidelines for Colorectal Cancer Survivors Based on Follow-Up Care Provider Specialty}

Devon Check, ${ }^{1}$ Neetu Chawla, ${ }^{2}$ Valerie Lee, ${ }^{1}$ Leslie Manace Brenman, ${ }^{1}$ Aileen De Mucha Flores, ${ }^{1}$ Raymond Liu, ${ }^{1}$ Yan Li, ${ }^{1}$ Annie Davis, ${ }^{1}$ Marilyn Kwan ${ }^{1}$

${ }^{1}$ Kaiser Permanente Northern California, Oakland, CA; ${ }^{2}$ Veterans Affairs Greater Los Angeles Healthcare System, Los Angeles, CA

Background: Guidelines recommend that patients with early-stage (I-III) colorectal cancer (CRC) receive regular posttreatment surveillance with colonoscopy, computed tomography (CT) scans, and carcinoembryonic (CEA) blood testing. Depending on patient's stage, completed treatment, preferences, and access, surveillance may be managed by a primary care provider (PCP) and/or a specialist. We investigated whether different providers' involvement in follow-up care may impact patients' receipt of guidelinerecommended surveillance.

Methods: We conducted a retrospective cohort study using data from Kaiser Permanente Northern California's cancer registry and electronic medical records. Patients in our sample $(\mathrm{N}=2,615)$ were diagnosed with stage I-III CRC from 2011 to 2014 , received surgery, and had at least 1 follow-up visit in the 6-18 months postdiagnosis. We created indicators of patients' visits with oncologists, surgeons, gastroenterologists, and PCPs. Using modified Poisson regression, we examined the association of each indicator with patients' receipt of $\geq 1$ colonoscopy (stages I-III), $\geq 1$ CEA test (stages II-III), and $\geq$ 1 chest, abdomen, or pelvis CT (stages II-III) during the same period. All models were adjusted for patient age, race, gender, comorbidity, tumor stage, site, and treatment received.

Results: In total, $67 \%$ of all patients saw an oncologist, $18 \%$ a surgeon, $16 \%$ a gastroenterologist, and $72 \%$ a PCP. In adjusted analyses, stage I-III patients who saw an oncologist were $38 \%$ more likely to receive a colonoscopy (adjusted risk ratio [aRR]: 1.38, 95\% CI: 1.21-1.55). Stage II-III patients seeing an oncologist were $129 \%$ more likely to receive CT (aRR: 2.29, 95\% CI: $1.93-2.72$ ) and $219 \%$ more likely to receive CEA testing (aRR: 3.19, 95\% CI: 2.72-3.78) compared to those who saw a different type of provider. Seeing a gastroenterologist versus a different type of provider was positively associated with colonoscopy receipt (aRR: 1.14, 95\% CI: 1.01-1.28). Contact with a PCP was not statistically significantly associated with receipt of any of these tests.

Conclusion: Patients seen by medical oncologists were more likely than those seen by other providers to receive each recommended surveillance test in the 6-18 months postdiagnosis. Our results highlight the need for further research on multilevel factors (eg, patient-provider relationships, provider familiarity with guidelines) that may explain our findings. They also highlight a potential need for interventions to improve surveillance for CRC survivors. Keywords: observational study, primary care, quality of care, clinical practice patterns/guidelines

P2.05

Incidence and Recurrence of Hepatocellular Carcinoma Associated With Oral Direct-Acting Antivirals

Sonal Singh, ${ }^{1}$ James Marshall, ${ }^{2}$ Kevin Haynes, ${ }^{3}$ Cheryl McMahill-Walraven, ${ }^{4}$ Jeffrey Brown ${ }^{2}$

${ }^{1}$ University of Massachusetts Medical School, Worcester, MA; ${ }^{2}$ Department of Population Medicine, Harvard Medical School and Harvard Pilgrim Health Care Institute, Boston, MA; ${ }^{3}$ HealthCore, Wilmington, DE; ${ }^{4}$ Healthagen Outcomes / Aetna, Blue Bell, PA 
Background: The influence of sustained virologic response induced by oral direct-acting antivirals on the risk of hepatocellular carcinoma (HCC) among patients with chronic hepatitis $\mathrm{C}$ is conflicting. Our objective was to determine the incidence and recurrence of HCC among adults with chronic hepatitis $\mathrm{C}$ who received oral directacting antivirals.

Methods: A retrospective cohort study among users of oral direct-acting antivirals from January 1, 2015, to December 31, 2016, in the NIH Collaboratory Distributed Research Network (DRN). The cohort included members $\geq 18$ years of age during the 2-year query period who had continuous drug and medical coverage for a minimum of 183 days. Incidence use was defined as no exposure to any of the directacting antivirals in the 90 days before the index dispensing. National Drug Codes were used to identify exposures and create the treatment episodes. The ICD-9-CM and ICD-10$\mathrm{CM}$ were used to identify the outcome of HCC. The users were required to have no preexisting $\mathrm{HCC}$ during the 180 days prior to the index date for the analysis of incident users. The analysis for recurrent $\mathrm{HCC}$ included incident users with preexisting $\mathrm{HCC}$ in the 366 days prior to incident use. Each data partner conducted its analysis in the local data environment and returned aggregate information to the $\mathrm{NIH}$ Collaboratory DRN Coordinating Center (Harvard Pilgrim Health Care Institute), which combined the data across sites. Results: There were 14,365,692 members eligible for the incident analysis and 4092 members eligible for the recurrent HCC analysis. Rates of new HCC for ombitasvir/paritaprevir/ ritonavir/dasabuvir (1.6\%), sofosbuvir/lepidasvir (11.9\%), sofosbuvir/veltapasvir ( $0 \%)$ and sofosbuvir (1.6\%) were uniformly low. Rates of recurrent HCC for sofosbuvir/ lepidasvir were high. Limitations include the small number of users for several drugs, absence of an unexposed arm, and confounding by disease severity and use of only 3 of the organizations that participate in the NIH Collaboratory DRN to minimize cost. Use of the full dataset would increase the number of users by approximately 8 -fold.

Conclusion: Sofosbuvir/lepidasvir was associated with low incidence of new-onset HCC. The persistence of recurrent HCC after treatment with oral direct-acting antivirals deserves further exploration.

Keywords: cancer, virtual data warehouse, clinical decisionmaking, epidemiology

\section{P2.06}

\section{Overall Ratings of Care Among Women Veterans With and Without a History of Cancer}

Neetu Chawla, ${ }^{1}$ Julian Brunner, ${ }^{1}$ Danielle Rose, ${ }^{1}$ Catherine Chanfreau, ${ }^{1}$ Jill Darling, ${ }^{2}$ Elizabeth Yano ${ }^{1}$

${ }^{1}$ Veterans Affairs Greater Los Angeles Healthcare System, Los Angeles, CA; ${ }^{2}$ University of Southern California, Los Angeles, $C A$
Background: As a minority group within the Veterans Affairs (VA) system, women veterans may experience unique challenges to accessing and coordinating health care services, such as identifying providers to manage and treat gender-specific health conditions or obtaining specialized services (eg, mammograms). Coordination of care is even more challenging for those with complex chronic conditions, such as cancer, which may require receipt of care outside the VA system. In this study, we examined how receipt of care outside VA impacts overall ratings of care among women veterans with and without a history of cancer.

Methods: We used patient-reported data from a crosssectional, weighted, telephone survey conducted between January and March 2015 at 12 VA medical centers among 1395 women veterans who had 3+ VA primary care visits in the prior year. We analyzed how women rated their overall health care on a $0-10$ scale and compared ratings between veterans with and without a history of cancer. Using linear regression, we modeled associations of care receipt outside the VA system with overall ratings of care among those with and without a history of cancer, controlling for sociodemographic and insurance characteristics. We ran an overall model in the full sample and stratified models among the cancer and noncancer populations.

Results: A majority of the sample was over 45 years old $(65 \%)$ or white race $(59 \%)$; nearly half reported having only VA coverage (42\%). Approximately $13 \%$ of the sample reported a history of cancer. In the overall model, we found that use of care from outside the VA system was associated with poorer ratings of care $(\mathrm{P}=0.018)$. However, when we stratified by cancer status, we saw that this association was only significant for the cancer population $(\mathrm{P}=0.001)$.

Conclusion: Among women veterans with a history of cancer, those receiving health care outside the VA system reported poorer overall care ratings versus those receiving care within the VA system alone. These findings may underscore care coordination challenges that exist for women veterans with cancer and point to the need for health care and policy interventions to improve coordination of care between VA and non-VA providers in the context of cancer care delivery.

Keywords: cancer, quality of care, VA health system, access to services

P2.07

Demographic, Clinical, and Utilization Factors Associated With Early-Age Pancreatic Cancer Diagnosis

David Mosen, ${ }^{1}$ Rajasekhara Mummadi, ${ }^{1}$ Matthew
Banegas, ${ }^{1}$ Elizabeth Shuster ${ }^{1}$

${ }^{1}$ Kaiser Permanente Northwest, Portland, OR

Background: Recent research has found increased incidence of pancreatic cancer among younger populations ( $\leq 50$ years old). Little is known about risk factors associated with pancreatic 
cancer diagnosis at an early age. This study examined demographic, clinical, and utilization factors associated with diagnosis at a younger age $(\leq 50)$ compared to older age $(>60)$. Methods: Using tumor registry data, we analyzed 17 years of pancreatic cancers diagnosed from January 1, 2000, to December 31, 2016, within Kaiser Permanente Northwest (KPNW), an integrated health system in the Pacific Northwest. This population was further divided into two age groups: younger age $(n=56)$ and older age $(n=810)$. Demographic, clinical, and utilization measures were identified via KPNW's electronic health record, including sex (female vs male), race/ethnicity (nonwhite vs white), smoking status (yes vs no), diabetes mellitus status (yes vs no), body mass index $(\mathrm{BMI} \geq 30$ vs $\mathrm{BMI}<30)$, and Charlson comorbidity index (CCI) score ( $1+$ vs $2+$ vs 0$)$, along with primary care visits, emergency department utilization, and hospital admissions in the 12 months prior to index diagnosis (1+ visits vs none). Multivariable logistic regression was used to measure the associations between these variables and the likelihood of pancreatic cancer diagnosis at an early age.

Results: We found a higher likelihood of being diagnosed with early-age pancreatic cancer: current smoker (odds ratio [OR]: 2.75, 95\% CI: 1.34-5.62), nonwhite ethnicity (OR: 3.61, 95\% CI: 1.79-7.28), BMI $\geq 30$ (OR: 1.89, 95\% CI: 1.02-3.53), and 1+ hospital admissions in the year prior to cancer diagnosis (OR: 2.07, 95\% CI: 1.05-4.11). A CCI score of $2+$ (OR: $0.40,95 \%$ CI: $0.19-0.84)$ and $1+$ primary care visits in the year prior to diagnosis (OR: $0.36,95 \% \mathrm{CI}$ : 0.13-0.96) were associated with a lower likelihood of earlyage pancreatic cancer diagnosis.

Conclusion: We found several demographic, clinical, and utilization factors associated with early-age diagnosis of pancreatic cancer. Specifically, the finding that nonwhites were more likely to be diagnosed at an early age deserves further exploration, with respect to key drivers explaining the finding. Moreover, future work is needed to understand how potentially modifiable factors like smoking status and obesity are linked to early-age pancreatic cancer diagnosis.

Keywords: observational study, demographics

\section{P2.08}

Care Coordination Experiences Among Women Veterans Diagnosed With Cancer and Co-Occurring Mental Health Conditions

\section{Neetu Chawla, ${ }^{1}$ Danielle Rose, ${ }^{1}$ Julian Brunner, ${ }^{1}$ Jill Darling, ${ }^{2}$ Elizabeth Yano ${ }^{1}$}

${ }^{1}$ Veterans Affairs Greater Los Angeles Healthcare System, Los Angeles, CA; ${ }^{2}$ University of Southern California, Los Angeles, $C A$

Background: Psychosocial distress is common among women diagnosed with cancer. The Institute of Medicine and Commission on Cancer have each emphasized the significance of addressing psychosocial health needs among cancer survivors and the importance of care coordination between providers to better manage patients' needs. The purpose of this study was to evaluate care coordination experiences among women veterans and examine whether a history of cancer and a co-occurring mental health condition was associated with poorer care receipt.

Methods: Data are from a cross-sectional, weighted, telephone survey of 1395 women veterans with $3+$ visits in the prior year. The survey was conducted between January and March 2015 at 12 VA medical centers with a response rate of $46 \%$. We examined associations of a history of cancer and a co-occurring mental health condition with the following self-reported outcomes: 1) primary care team managed care between providers poorly; 2) care coordination was difficult between VA and non-VA providers; and 3) care coordination was difficult between primary and specialty care within the VA. In multivariable regression analyses, we controlled for sociodemographic and insurance characteristics and ran separate models for women with a history of cancer and depression and women with a history of cancer and anxiety. Results: A majority of the sample was over 45 years old $(65 \%)$ or white race $(59 \%)$; nearly half reported having only VA coverage $(42 \%)$. In evaluating care management experiences, we found that women with depression (odds ratio [OR]: 2.11, 95\% CI: 1.56-2.83) and women with anxiety (OR: 2.08, 95\% CI: 1.47-2.94) reported significantly poorer care. Women with anxiety also reported significantly poorer coordination experiences between VA and nonVA providers (OR: 2.11, 95\% CI: 1.31-3.40). Women with cancer reported poorer care for both outcomes but findings were not significant. Experiences of coordination between VA primary and specialty care providers were not significantly different for any of these groups.

Conclusion: Our results indicate that women with cancer and a co-occurring mental health condition have poorer care coordination experiences, primarily driven by their mental health status. Screening and treating psychosocial distress among women veterans may help to improve patient care experiences, while further investigation is needed to examine the needs of cancer patients within the VA system.

Keywords: cancer, quality of care, VA health system, patient experience/satisfaction

\section{P2.09}

Enhancing Virtual Data Warehouse Cancer Data: An Integrated, Open-Access System's Efforts

Pamala A. Pawloski, ${ }^{1}$ Jody Jackson, ${ }^{1}$ Terese A. DeFor, ${ }^{1}$ Amy L. Butani, ${ }^{1}$ Carla Saxen, ${ }^{1}$ Sheryl Kane ${ }^{1}$

${ }^{1}$ HealthPartners Institute, Bloomington, $M N$

Background: HealthPartners (HP) and Park Nicollet (PN) merged in 2014 to form one multispecialty care group with a cohesive electronic health record (EHR). This unique, integrated, open-access structure provides HealthPartners 
Institute (HPI) access to large populations of members, patients, and patient-members to enhance the virtual data warehouse (VDW). HPI has undertaken efforts to augment its VDW, including incorporating additional cancer cases by gaining access to additional owned sites, refining staging information, and adding new analytic data variables.

Methods: Tumor data from two PN hospitals, Methodist and St. Francis, were incorporated into the VDW. Through collaborations with the Hospital Tumor Registry, mapping of ERS variables to North American Association of Central Registries variables was reviewed to ensure comprehensive identification of staging information. We subsequently developed a new method for obtaining American Joint Committee on Cancer (AJCC) stage information. This method was validated via chart review on a sample of 27 colorectal cancer (CRC) cases. Clinical record linkages to identify PN patients have been completed across the integrated EHR. Additional VDW enhancements include the inclusion of chemotherapy infusion data and laboratory values including prostate-specific antigen (PSA), alphafetoprotein (AFP), cancer antigens 19-9 (CA 19-9) and 125 (CA 125), and carcinoembryonic antigen (CEA).

Results: With the inclusion of the additional hospital data, our VDW tumor table now includes 51,207 total cancer cases (increased from 25,924 available in 2016), including 11,453 breast, 3129 colorectal, 5849 lung, and 4867 prostate cancer cases. Results of the staging validation indicated concordance between the EHR and the new AJCC stage method for $81 \%$ (22 of 27) of the CRC cases. Following the addition of PN, HPI data now includes over 6 million patients whose utilization will be added to the VDW. In addition, data on more than 900,000 administered infusions and 15,000 PSA, 49,467 AFP, 13,288 CA 19-9, 23,094 CA 125 , and 302 CEA values are available in the VDW.

Conclusion: With the expansion of our VDW, we have doubled our cancer cases, increased the accuracy of staging data, included data regarding infusions administered and laboratory values, and will be incorporating additional EHR utilization. These additions greatly expand our capabilities for performing cancer research.

Keywords: virtual data warehouse

\section{P2.10}

\section{Cisplatin-Associated Ototoxicity May Affect Patient Adherence to Cancer Treatment}

\section{Steven Chang ${ }^{1}$}

\section{${ }^{1}$ Henry Ford Health System, Detroit, MI}

Background: Cisplatin is a common and effective drug used in the management of numerous malignancies. A number of studies have reported on the ototoxic profile of cisplatin; however, no study has analyzed the impact of cisplatinassociated ototoxicity on patient completion of a prescribed cisplatin-based chemotherapy regimen. This study aimed to assess the relationship of cisplatin-associated ototoxicity on patient completion of a cisplatin-based chemotherapy regimen at a single academic institution.

Methods: A retrospective database of patients with head and neck cancer was queried for those treated with chemotherapy during 2013-2016. Patients who received cisplatin during their treatment were included in this study. Statistical analysis was used to study the association of cisplatin-associated ototoxicity on patient completion of the prescribed chemotherapy regimen.

Results: A total of 193 patients were included in the database. Average age was 61.39 years (SD: 12.30 ), and $79.8 \%$ of patients were male. A total of 103 patients (53.4\%) were prescribed cisplatin as a treatment modality. Pre-, mid- and post-chemotherapy treatment audiograms were recommended for $68.9 \%$ of patients receiving cisplatin therapy; however, $3.9 \%(n=4)$ completed pretreatment audiograms, 8.7\% $(n=9)$ completed midtreatment audiograms, and 19.4\% ( $\mathrm{n}=20)$ completed posttreatment audiograms. Among patients on cisplatin treatment, $14.6 \%(n=15)$ reported otologic concerns at 3-month follow-up compared to $2.2 \%(\mathrm{n}=2)$ on noncisplatin chemotherapy. In analyzing adherence to therapy, $4.9 \%(n=5)$ of patients on cisplatin treatment switched their chemotherapy regimen due to ototoxicity, while $12.6 \%(n=13)$ switched their chemotherapy regimen due to nonototoxic reasons. Comparatively, of those undergoing noncisplatin therapy, none switched their chemotherapy due to ototoxicity while $11.1 \%$ (10) switched due to nonototoxic reasons.

Conclusion: Cisplatin-associated ototoxicity is associated with noncompletion of a prescribed chemotherapy regimen in some patients. Further research is needed into this area, as the ototoxic effects are likely to have impact on patient quality of life in survivorship.

Keywords: aging, elderly, geriatrics, cancer, quality of life, quality of care, clinical practice patterns/guidelines, quality improvement

\section{CARE IMPROVEMENT}

\section{P4.01}

Local Implementation of Cancer Care Delivery Research: S1417CD Impact of Cancer on Finances

\section{Pamala A. Pawloski, ${ }^{1}$ Heather Kehn, ${ }^{2}$ Alice Shapiro, ${ }^{3}$ Daniel Anderson, ${ }^{4}$ Michele Lacy, ${ }^{2}$ Veena Shankaran ${ }^{5}$}

${ }^{1}$ HealthPartners Institute, Bloomington, $M N ;{ }^{2}$ MetroMinnesota Community Oncology Research Consortium, St. Louis Park, MN; ${ }^{3}$ Frauenshuh Cancer Center, St. Louis Park, MN; ${ }^{4}$ Regions Hospital, Saint Paul, MN; ${ }^{5}$ Fred Hutchinson Cancer Research Center, Seattle, WA

Background: A cancer diagnosis can carry enormous personal financial hardship. Patients frequently struggle with finances and make tough decisions. To understand the 
personal financial toll from cancer, our National Cancer Institute (NCI) Community Oncology Research Program is participating in the NCI-funded cancer care delivery research study "Implementation of a Prospective Financial Impact Assessment Tool in Patients with Metastatic Colorectal Cancer" (S1417CD). The ultimate goal of this research is to measure the association between cancer treatment and financial hardship, using a comprehensive financial impact assessment tool that can be adopted in routine clinical care and alongside cooperative group clinical trials. Challenges anticipated include a lack of knowledge of financial terminology, reluctance to discuss financial details, and health professionals being unprepared to discuss financial matters.

Methods: S1417CD will assess the incidence of treatmentrelated major financial hardship over 12 months following a diagnosis of metastatic colorectal cancer. Patients will complete questionnaires at baseline and every 3 months regarding the impact of cancer on their financial situation. Data regarding credit attributes will be collected by a third party. Our local study implementation plan included engaging community programs and advocacy networks to understand how best to discuss this sensitive topic and incorporating a variety of communication vehicles targeting the general public, research community, and providers. We leveraged local newspaper and television news, a regional webinar with the American Cancer Society, and a local physician magazine.

Results: Our institutional review board approved the study in March 2017. Staff training and site implementation occurred in April 2017, and the study was activated locally in May 2017 at 5 component sites. As of October 2017, 10 local component sites are enrolling. To date, 13 participants have been enrolled locally and 117 nationally. There are 470 study locations nationally and the total targeted enrollment is 374 .

Conclusion: The successful local implementation of this study is the result of our strong local infrastructure and the salience of financial toxicity research. In addition, the concerted effort to communicate the importance of this research and the availability of the study through a variety of communication vehicles targeted to multidisciplinary audiences enhanced our recruitment and enrollment efforts. Keywords: cancer, quality of life, health care costs, resource use

\section{P4.02}

Exploring Patient and Health Care Professional Experience With the Medication Prior Authorization Process Within a Rural Health Care Setting

\author{
Laney Jones, ${ }^{1}$ llene Ladd, ${ }^{1}$ Jove Graham, ${ }^{1}$ Michael \\ Evans, ${ }^{1}$ Michael Gionfriddo ${ }^{1}$ \\ ${ }^{1}$ Geisinger Health, Danville, $P A$
}

Background: The number of medications requiring prior authorization (PA) is projected to increase over the next decade due to increased use of high-cost medications (eg, biologics) for previously untreated or targeted conditions. PA has been shown to be burdensome for health care professionals; little is known, however, about the impact of the PA process on patient experience.

Methods: Semi-structured interviews were conducted with patients, and a focus group was conducted with health care professionals. Patients who had received a medication requiring PA and who had been seen at Geisinger Health's Wilkes-Barre Rheumatology Clinic, as well as health care professionals who work at that clinic, were recruited by study team. Participants were asked about their experience with the current PA process. Recordings were transcribed verbatim and reviewed for accuracy and completeness. Two reviewers independently reviewed the transcripts and coded the data. Consensus coding was then completed and relevant themes were extracted.

Results: Seven patients shared their experience with receiving a medication that required PA. Most patients felt that insurance companies imposing restrictions on access to medications caused a delay in their care and that the process was confusing and unfriendly. Overall, patients had positive experiences with their doctors and pharmacies when being prescribed a medication that required PA but did express concerns when learning about high costs of their medications. Ten medical staff within the rheumatology department were asked to give feedback on the appropriateness and usefulness of survey questions regarding the PA process. Individuals had varying perspectives based on their level of engagement with the PA process, but all felt that a team approach was necessary to provide high-quality care for their patients. Many expressed uncertainty in knowing which medications require PA and perceived that this process delays care for their patients. Physicians felt that individuals making PA decisions at some health insurance companies did not have the medical expertise required.

Conclusion: This study provides insights into the patient's experience when obtaining medications that require PA and expands the current literature on health care professional experience. In addition, learnings will be used to impact implementation of new PA processes.

Keywords: qualitative research

P4.03

Advancements in Care Coordination: Findings From an Evaluation of Minnesota's State Innovation Model Initiative

Christina Worrall, ${ }^{1}$ Donna Spencer, ${ }^{2}$ Caroline AuYeung, ${ }^{1}$ Emily Zylla, ${ }^{1}$ Kelli Johnson, ${ }^{1}$ Lacey Hartman ${ }^{1}$

${ }^{1}$ State Health Access Data Assistance Center, Minneapolis, $M N{ }^{2}$ The Lewin Group, Eden Prairie, MN

Background: Between 2013 and 2017, under a federal State Innovation Models (SIM) cooperative agreement, 
Minnesota implemented a model for transforming the state's health care payment and service delivery systems in order to lower costs, increase quality, and improve population health. One aim of Minnesota's SIM model was that the majority of individuals would receive care that is patient-centered and coordinated across settings. This aim underpinned four of the state's SIM strategies: 1) advance providers' health information exchange (HIE) ability; 2) increase provider access to data analytics; 3 ) implement practice transformation to expand the number of patients receiving team-based integrated/coordinated care; and 4) increase partnership among organizations and individuals to create Accountable Communities for Health (ACHs). Under a contract with the state, external researchers examined whether and how these strategies increased the capacity of participating organizations to deliver coordinated care.

Methods: This analysis was part of a broader evaluation of Minnesota's SIM model conducted from 2015 through 2017 using both quantitative and qualitative methods. Key data sources included semi-structured interviews with organizations participating in SIM and with the state, a SIM organization survey, an ACH provider survey, and a survey of HIE users under SIM.

Results: Interview and survey data indicated, broadly, an increased capacity to deliver coordinated care across medical and nonmedical settings, which interviewees attributed largely to strengthened relationships with, and knowledge of, other providers, organizations, and resources. Data also showed, to a varying extent, improvement in select care coordination processes/capabilities (eg, assessments of social determinants). Where growth was not seen, data indicated that SIM participants came into the initiative with capabilities. SIM participants reported an increase in access to or collection of data to improve care/service coordination. Providers participating in $\mathrm{ACH}$ care coordination models reported positive impacts on workload and workflow.

Conclusion: This analysis found an increased capacity to coordinate care among organizations participating in SIM in Minnesota. Future efforts to enhance this capacity should work to demonstrate the value of care coordination with a population health focus while also targeting organizations that are less developed, encouraging community connections, and prioritizing investments in HIE and data analytics to support care coordination.

Keywords: program evaluation, referrals, networks, quality of care, state health policies, evaluation research, health policy/regulation, social determinants of health, Medicaid/ SCHIP, qualitative research, quality improvement, patient experience/satisfaction
P4.04

The Underpads Study: Safe Delivery of Patient Output Without the Risk of Catheter-Associated Urinary Tract Infection

\section{Kealy $\operatorname{Ham}^{1}$ \\ ${ }^{1}$ HealthPartners Medical Group / Regions Hospital / University of Minnesota, Saint Paul, MN}

Background: Historically, the indwelling catheter has been an automatic accessory in critically ill patients to measure accurate input and output measurements. Catheterassociated urinary tract infections (CAUTI) can be decreased or eliminated by using simple scales and weighing of underpads for adult patients in the hospital. By not inserting an indwelling catheter, the risk of infection is eliminated. We developed an alternative quality improvement plan using underpads instead of indwelling catheters to capture the same data in a safer way for our patients. Department-based standard-of-care procedures may be improved by measuring fluid accumulation or volume loss in patients with this novel method.

Methods: On the medical intensive care unit in our urban Level I trauma hospital, we identified patients who required input and output data who were admitted with a diagnosis of alcohol use/abuse, polysubstance use/abuse, ingestion, or toxic encephalopathy. Patients were eligible if they were not on diuretic therapy, did not have wounds at risk for contamination, and did not have a history of urinary retention. If the patient had an existing indwelling catheter, it was removed. If the patient did not have one, none was placed. A simple metric-scale weight was obtained and the clean pad was placed under the patient. The pad was checked every 2 hours as is standard for our patient-positioning strategy. Any wet pad was immediately removed from the patient and the standard cleaning and skin protection process was followed. The weight of the pad was documented as milliliters of urine (or mixed) output.

Results: There was a $68 \%$ reduction in duration of indwelling catheter time in patients with underpad use versus routine care. There was a decrease in CAUTI rates compared to pretrial and the national rate. There was no incidence of pressure ulcers in either group.

Conclusion: The evaluation of the use of underpads instead of indwelling catheters challenges common practices and questions why we continue to use practices that may lead to harm. This may result in safer patient care, improved quality of care, and elimination of potential financial consequences from publicly reportable infection data.

Keywords: acute inpatient care, health care costs, resource use, hospital, patient safety, quality improvement 
P4.05

Variation in Patterns of Care Related to Control of Cardiovascular Risk Factors Across Primary Care Providers

Jeffrey Anderson, ${ }^{1}$ JoAnn Sperl-Hillen, ${ }^{1}$ A. Lauren Crain, ${ }^{1}$ Jay Desai, ${ }^{1}$ Karen L. Margolis, ${ }^{1}$ Heidi L. Ekstrom, ${ }^{1}$ Patrick J. O'Connor ${ }^{1}$

\section{${ }^{1}$ HealthPartners Institute, Bloomington, MN}

Background: We used output from a primary care clinical decision support system to investigate the degree of variation across primary care providers (PCPs) in firing frequency of two treatment recommendations related to aspirin use for cardiovascular prevention and screening for diabetes.

Methods: We identified 12,234 adults (with high 10-year cardiovascular disease risk but without diabetes or heart disease) under the care of 161 PCPs (range: 7-494 eligible patients), in a subset of a larger randomized trial (HL102144). We used descriptive statistics to summarize the distribution of PCP-specific proportions of patients for whom these selected treatment recommendations fired. Additionally, we stratified these distributions by the proportion of each PCP's patients who were insured by Medicaid to evaluate whether observed variation in treatment recommendations could be explained by differences in socioeconomic status across provider-specific patient populations. Statistical comparisons were done using Wilcoxon rank-sum tests. We also computed $r^{2}$ values using linear regression, which indicate the amount of variation in care recommendation fire rates that could be explained by Medicaid distribution.

Results: The concurrent nonsteroidal anti-inflammatory drug and aspirin use safety recommendation fired for $21.5 \%$ of patients of the median PCP (10th percentile: 10.0\%; 90th percentile: $36.1 \%$ ). The frequency of this recommendation was significantly higher for PCPs with a greater proportion of patients on Medicaid (median of $\geq 5 \%$ vs $<5 \%$ on Medicaid: $25.0 \%$ vs $19.7 \% ; \mathrm{P}=0.026$ ). However, variation in the frequency of this recommendation was substantial regardless of Medicaid status $\left(\geq 5 \%\right.$ vs $<5 \%$ on Medicaid for $10^{\text {th }}$ percentile: $11.1 \%$ vs $10.2 \%$; 90 th percentile: $38.6 \%$ vs $30.8 \%$; $\mathrm{r}^{2}=0.032$ ). The diabetes screening recommendation fired for $28.0 \%$ of patients of the median PCP and varied across PCPs (10th percentile: $15.8 \%$; 90 th percentile: $39.5 \%$ ). The rate of this recommendation firing did not differ by proportion of PCP's patients on Medicaid $\left(\mathrm{P}=0.631 ; \mathrm{r}^{2}=0.003\right)$.

Conclusion: These data demonstrate substantial variability across primary care providers in key safety and care practice patterns. The results suggest an opportunity to design PCP interventions based on PCP-specific care improvement opportunities that can be identified through clinical decision support systems.

Keywords: cardiovascular disease, ambulatory/outpatient care, observational study, quality of care, diabetes, Medicaid/ SCHIP, chronic disease, clinical practice patterns/guidelines
P4.06

PACT - A Self-Management Program for Chronic Pain Utilizing Online Education and Telehealth Coaching: Findings of the Pilot Study

\author{
Elizabeth Grossman, ${ }^{1}$ Gabriela Vazquez-Benitez, ${ }^{1}$ \\ Robin Whitebird, ${ }^{2}$ Karen Lawson, ${ }^{3}$ James Fricton ${ }^{1}$
}

${ }^{1}$ HealthPartners Institute, Bloomington, MN; ${ }^{2}$ University of St. Thomas, Minneapolis, $M N ;{ }^{3}$ consultant (unaffiliated)

Background: Temporomandibular disorders (TMD) are one the most common pain conditions, affecting more than $10 \%$ of the general population. The Personalized Activated Care and Training (PACT) program provides patients with a telehealth coach and online self-management modules using cognitive-behavioral strategies to train patients to reduce risk factors and implement protective actions that can reduce chronic pain. Our focus of this analysis was to evaluate the use of telehealth coaching among those participants in the PACT online program.

Methods: Data were gathered from participants enrolled in the TMD self-care pilot randomized clinical trial. The pilot compared the PACT program to traditional self-care for TMD pain. To understand the impact of the telehealth coach in the online self-management program, we examined participant compliance with the telehealth coach model. Within the PACT arm, we identified participants who engaged in 3 or more sessions with coaches (high engagement). We examined their baseline characteristics, usability of the online program, and change in chronic pain self-efficacy at 8-week follow-up. Participant feedback was captured from program evaluation, and telehealth coaching experience was obtained from phone interviews.

Results: Among the 40 subjects who were randomized to the PACT program, $27(69 \%)$ demonstrated high engagement in health coaching. Characteristics associated with higher engagement were being female, age 30-65 years, experienced chronic pain for 3 or more years, having a TMD screen score of 5 points, and self-efficacy. Highly engaged participants had better adherence to the online program and had lower change in self-efficacy $(6.5 \%$ vs $36.26 \%$ ). Based on interviews regarding their experiences with health coaching, highly engaged participants reported that "She helped me set goals; her support was great" and "It was a way to make sure that I stayed on track" and "I used it as part of my self-management."

Conclusion: For participants with chronic pain, telehealth coaching is a key component of the online self-management PACT program. Those who have a high level of engagement are more likely to adhere to the program and have a positive perception of their health coach in supporting selfmanagement of pain.

Keywords: information technology, quality of care, health promotion, prevention, screening, dental care, patient experience/satisfaction 
P4.07

\section{Prehospital Lactate: A Severity Indicator in Early Sepsis Management}

Kurt Isenberger, ${ }^{1}$ Aaron Burnett, ${ }^{1}$ Jeffrey Anderson, ${ }^{2}$ Adam Mayer, ${ }^{1}$ Sandi Wewerka, ${ }^{1}$ Joseph Pasquarella, ${ }^{1}$ Ralph Frascone ${ }^{1}$

\section{${ }^{1}$ Regions Hospital, Saint Paul, MN; ${ }^{2}$ HealthPartners Institute, Saint Paul, MN}

Background: Serum lactate levels can rapidly dictate clinical awareness of shock and prompt intervention in sepsis. Collecting serum lactate levels is restricted to the hospital setting. The objective of this study was to investigate whether a prehospital lactate (PL) measurement improves time to intervention upon emergency department (ED) arrival. We hypothesized that emergency medical services communication of a PL value to the ED physician would result in improved time to antibiotics and an in-hospital lactate order.

Methods: This prospective, observational study included patients with a prehospital impression of infection based on systemic inflammatory response syndrome criteria. A pointof-care testing PL was collected by paramedics prior to ED arrival and reported during presentation. ED metrics were collected and compared to a previously collected control group of patients presenting without a PL but who met early sepsis criteria. Cox regression models were used to estimate hazard ratios with $95 \%$ confidence intervals for time to physician order for an antibiotic or in-hospital lactate test.

Results: Study patients (60-96 years old) included 170 with PL measures and 269 controls. The PL group was older on average (mean age: 69 vs 54 years, $\mathrm{P}<0.001$ ) and was more likely to have expired in the hospital $(10 \%$ vs $5 \%, \mathrm{P}=0.027)$. An antibiotic was ordered for $104 \mathrm{PL}$ patients $(61 \%)$ and 216 controls $(80 \%)$. In a Cox model adjusted for age and gender, the control group had a nearly 2-fold faster rate of time to antibiotic order (hazard ratio: 1.93, 95\% CI: $1.50-2.48$ ), relative to PL patients. Among the PL patients, time to antibiotic order did not differ by PL ( $>2$ vs $\leq 2 \mathrm{mmol} / \mathrm{L}, \mathrm{P}=0.545$ ). Time to in-hospital lactate order did not significantly differ between control group and patients with $\mathrm{PL}>2 \mathrm{mmol} / \mathrm{L}(\mathrm{P}=0.811)$, but time to inhospital lactate order was twice as slow for patients with $\mathrm{PL} \leq 2 \mathrm{mmol} / \mathrm{L}$ (hazard ratio (vs control group): $0.47,95 \%$ CI: 0.33-0.68).

Conclusion: In this small study, the availability of PL value did not improve time to antibiotics. Patients with a normal PL had a prolonged time to antibiotics compared to the control group, and a higher death rate. A larger study is required to validate these results.

Keywords: acute inpatient care, clinical decision-making, clinical practice patterns, guidelines
P4.08

\section{Encouraging Patients to Speak Up About Breakdowns in Care}

Kathleen M. Mazor, ${ }^{1}$ Kelly M. Smith, ${ }^{2}$ Thomas $H$. Gallagher, ${ }^{3}$ Sybil Crawford, ${ }^{4}$ Yanhua Zhou, ${ }^{1}$ Azraa Amroze, ${ }^{1}$ Kimberly A. Fisher ${ }^{4}$

${ }^{1}$ Meyers Primary Care Institute, Worcester, MA; ${ }^{2}$ MedStar Health Research Institute, Hyattsville, MD; ${ }^{3}$ University of Washington Medical Center, Seattle, WA; ${ }^{4}$ University of Massachusetts Medical School, Worcester, MA

Background: As many as $40 \%$ of hospitalized patients perceive a breakdown in their care, but many are reluctant to speak up, resulting in missed opportunities to improve care. Strategies to encourage patients to speak up are needed.

Methods: We surveyed a national sample of adults age > 35 years using an online, vignette-based questionnaire. Participants were asked to imagine experiencing 3 common breakdowns during hospitalization: slow response to the call bell; rudeness on the part of the aide; and having unanswered questions. Participants were randomly presented with one of 9 versions of a brief conversation that might prompt a patient to speak up. Conversations described a doctor, nurse, or patient specialist who provided a minimal, intermediate, or extended prompt to speak up. Participants rated how upset they would be about each breakdown, whether they would speak up about each breakdown, and whether they would recommend the hospital.

Results: The final sample size was 1188 (response rate: $64.4 \%$ ). Overall, participants were most upset about having unanswered questions $(84.7 \%$ would be extremely/very upset), least upset about slow response to the call bell (35.4\% extremely/very upset), with rudeness of the aide in between (66.3\% extremely/very upset). The inquirer's role affected whether participants would report the slow response to the call bell (23.4\% definitely would tell the physician, $27.7 \%$ the nurse, $42.7 \%$ the patient specialist; $\mathrm{P} \leq 0.0001)$ and rudeness (34.2\% would definitely tell the physician, $40.3 \%$ the nurse, $54.5 \%$ the patient specialist; $\mathrm{P} \leq 0.0001$ ) but not intent to speak up about unanswered questions $(80.7 \%$ would definitely speak up overall; $\mathrm{P}>0.10$ ). We found no impact of wording on intent to speak up ( $\mathrm{P}>0.10)$, but wording did affect willingness to recommend the hospital; $18.4 \%$ would probably or definitely recommend when given an extended prompt, compared to $14.6 \%$ and $8.8 \%$ for the intermediate and minimal prompts, respectively $(\mathrm{P}=0.0067)$.

Conclusion: Knowing when patients and family members perceive a breakdown in care is the first step in addressing their concerns and may help to improve care for future patients. The findings from this national survey suggest that the type of breakdown experienced and the person inquiring about care influence whether patients will speak up.

Keywords: survey research and methods, patient-provider communication, patient experience/satisfaction 


\section{P4.09 \\ Moving the Needle: Is Adjusting the Needle Between Endobronchial Ultrasound Passes Necessary?}

\section{David Niccum ${ }^{1}$}

\section{${ }^{1}$ Regions Hospital, Saint Paul, MN}

Background: Endobronchial ultrasound (EBUS) with transbronchial needle aspiration biopsy is effective for diagnosing multiple thoracic pathologies and staging lung cancer. In EBUS, a biopsy needle is inserted through the working channel of an EBUS bronchoscope and directed via linear ultrasound into a suspected lymph node or lesion. Multiple passes increase the probability of adequate specimen retrieval. A protective sheath surrounds the biopsy needle, protecting the working channel of the EBUS bronchoscope from damage during the procedure. Prior to the biopsy, the sheath is adjusted to the length of the working channel. Some manufacturers recommend readjustment after each pass. Readjustment requires additional time, potentially prolonging sedation time and patient discomfort. Previous studies addressing needle repositioning were not identified. This study sought to determine whether readjustment is necessary.

Methods: A total of 29 sequential passes were performed with an Olympus ViziShot NA201SX4021 needle and Olympus UC160F-OL8 EBUS bronchoscope; 10 passes were performed in a neutral position, followed by 10 passes alternating between fully flexed and extended, and 9 with the bronchoscope flexed or extended into simulated tissue. The position of the catheter in relation to the bronchoscope was measured and photographed after each pass.

Results: After the 29th pass, the spring guarding the needle dislodged from the sheath, resulting in structural failure. This form of sheath failure has been reported previously. The maximal deviation of the sheath was $1.5 \mathrm{~mm}$ beyond the end of the working channel and occurred twice. No inward sheath deviation occurred beyond the initial position on any pass. On the final pass, the sheath was $0.5 \mathrm{~mm}$ beyond the initial position.

Conclusion: Minimal variation of the position of the biopsy needle in relation to the bronchoscope between passes was observed. As none of the passes demonstrated inward deviation of the sheath into the working channel, it is unlikely the slight deviation observed would damage the bronchoscope. Since concluding this study, our group has adopted the policy that needle readjustment is not required between passes. While there is potential for widespread adoption of this practice, additional study is needed on other biopsy needles to determine whether these observations are true for all biopsy needles.

Keywords: quasi-experiment, clinical practice patterns, guidelines, quality improvement

\section{CHILD AND ADOLESCENT HEALTH}

\section{P5.01 \\ Elevated Blood Pressure and Risks for Progression to Hypertension in Youth 10 to 17 Years of Age}

Elyse O. Kharbanda, ${ }^{1}$ Stephen E. Asche,${ }^{1}$ Alan R. Sinaiko, ${ }^{2}$ James D. Nordin, ${ }^{1}$ Heidi L. Ekstrom, ${ }^{1}$ Steven P. Dehmer ${ }^{1}$

${ }^{1}$ HealthPartners Institute, Minneapolis, $M N ;{ }^{2}$ University of Minnesota, Minneapolis, MN

Background: Elevated blood pressure (BP) in children and adolescents is defined as having 1 or more BP measurements $\geq 90$ th percentile or $\geq 120 / 80 \mathrm{mmHg}$. Prior studies have reported annual risks for elevated BP to progress to hypertension as high as 7\%. The goal of the current study, conducted in a large health system, was to evaluate risk for progression to hypertension among youth with elevated BP confirmed by 2 or more elevated BPs.

Methods: All BPs, heights, and weights measured in 10-17-year-olds receiving care in one of 21 primary care clinics in a large Midwestern health system from April 15, 2014, to April 14, 2017, were pulled from the electronic health record. Using methods specified in the fourth report by the National High Blood Pressure Education Program Working Group on Children and Adolescents, BP percentiles were calculated. Patients with at least 2 elevated BPs within the initial 2 years and at least 2 subsequent BP measurements within 12 months were retained in the cohort. Hypertension was defined as having 2 or more BPs $\geq 95$ th percentile at consecutive health care visits. Covariates included race/ethnicity, age, gender, and body mass index (BMI) at date of second elevated BP. Associations were tested with Pearson's chi-squared and Fisher's exact tests. Multivariable logistic regression predicted progression to hypertension from sex and BMI $(\geq 95 \%$ vs $<95 \%)$.

Results: Of 31,579 patients with at least one BP recorded over a 2-year period, $5306(17 \%)$ had 2 or more elevated BPs and 1821 (34\% of those with persistent elevated BP) had 2 or more BPs measured in the subsequent 12 months. Among 1821 youth with persistent elevated BP and followup available, $46(2.5 \%)$ progressed to meet criteria for hypertension. Rates for progression were directionally higher for females ( $3.3 \%$ vs $1.9 \%$ in males, $\mathrm{P}=0.06)$, higher for youth with obesity (defined as BMI $\geq 95$ th percentile) (4.6\% vs $1.7 \%$ in nonobese youth, $\mathrm{P} \leq 0.001)$. In the multivariable model, progression to hypertension differed by sex $(\mathrm{P}=0.03)$ and $\mathrm{BMI}(\mathrm{P}<0.001)$. No pairwise interactions were significant in this model.

Conclusion: Persistent elevated BP in children and adolescents had a mild-moderate annual risk for developing hypertension of $2.5 \%$. Interventions to promote exercise and weight loss are indicated for youth with persistent elevated $\mathrm{BP}$ and obesity, as they are at highest risk for progression.

Keywords: child and adolescent health 
P5.02

\section{Effectiveness of Maternal Tdap Administration on Pertussis Disease During an Infant's First Year of Life}

\section{Malini DeSilva, ${ }^{1}$ Gabriela Vazquez-Benitez, ${ }^{2}$ Elyse O. Kharbanda ${ }^{2}$}

\section{${ }^{1}$ Minnesota Department of Health, Saint Paul, MN; ${ }^{2}$ HealthPartners Institute, Bloomington, MN}

Background: Since late 2012, the tetanus, diphtheria, and acellular pertussis (Tdap) vaccine has been recommended for routine administration during pregnancy to protect infants from pertussis disease. Few studies have evaluated the effectiveness of this immunization practice on infant pertussis. We aimed to determine the vaccine effectiveness of maternal Tdap at preventing pertussis disease in infants.

Methods: We conducted a matched case-control study of infants age $<365$ days from January 1, 2012, to June 30, 2012, with diagnostic code (ie, ICD-9 or ICD-10) or laboratory result positive for pertussis. We matched controls based on age (birthdate within 2 weeks of case), number of infant diphtheria, tetanus, and pertussis (DTaP) vaccinations received, and primary care clinic location for HealthPartners patients. We attempted to contact caregivers to enroll participants and then administer a survey to verify pertussis disease, determine maternal Tdap vaccination status, identify potential covariates/ confounders, and obtain consent for medical chart review. We used the Council of State and Territorial Epidemiologists case definition for probable and confirmed pertussis. We calculated matched odds ratios for the association between maternal vaccination and infant pertussis disease. We calculated vaccine effectiveness as 1.0 minus the odds ratio.

Results: We identified 172 cases, enrolled 76 (44\%), and performed chart reviews on $53(31 \%)$. Polymerase chain reaction testing was performed for 51 of $53(96 \%)$ and positive for 27 of 51 (53\%); we classified 11 of $53(21 \%)$ as confirmed and 21 of $53(40 \%)$ as probable pertussis. A total of 31 cases were matched to at least 1 control (range: $1-3)$; only matched cases contributed to analysis. Mothers of $10(32 \%)$ case patients and $23(40 \%)$ control patients received pertussis vaccination during pregnancy (unadjusted odds ratio: $0.76,95 \% \mathrm{CI}$ : 0.31-1.95), providing vaccine effectiveness of $24 \%$.

Conclusion: We did not find a significant decrease in odds of pertussis disease in infants whose mothers received Tdap during pregnancy compared to those with mothers who did not receive Tdap. A small response rate, resulting in a lower than anticipated sample size, likely contributed to our results. Additionally, nonresponse bias may have biased our results toward the null, as those we were unable to contact may have more unstable lives and would be less likely to adhere to health recommendations.

Keywords: child and adolescent health, human subjects research, survey research and methods, primary care, ambulatory/outpatient care, epidemiology
P5.03

\section{Utilization of and Primary Care Physician Experience With a Behavioral Health Navigation Program for Adolescent Patients}

Martina Li, ${ }^{1}$ Ellis Dillon, ${ }^{1}$ Jinnan $\mathrm{Li}^{1}{ }^{1}$ Yan Yang, ${ }^{1}$ Kimberly Erlich, ${ }^{2}$ Amy Heneghan, ${ }^{3}$ Ming Tai-Seale, ${ }^{3,4}$ Daniel Becker²

${ }^{1}$ Palo Alto Medical Foundation Research Institute, Sutter Health, Mountain View, CA; ${ }^{2}$ Mills-Peninsula Health Services, Sutter Health, San Mateo, CA, ${ }^{3}$ Palo Alto Medical Foundation, Sutter Health, Palo Alto, CA; ${ }^{4}$ University of California San Diego Health, San Diego, CA

Background: We are studying the utilization and physician experience of a navigation program within an adolescent behavioral health $(\mathrm{BH})$ quality improvement project in a multispecialty practice setting. The navigation program of this project was developed to support primary care physicians (PCPs) and to improve the quality of $\mathrm{BH}$ care by facilitating adolescent referrals from PCPs to $\mathrm{BH}$ specialists. Methods: Navigation referral data were analyzed to assess service utilization. Pediatric and family medicine PCPs were surveyed about their experience with the navigation program. Survey collection is ongoing and results will be updated.

Results: Within a 30-month period, 313 PCPs referred 3211 adolescent patients to the navigation program. The top three reasons for referral were depression $(37.2 \%)$, anxiety (24.1\%), and attention disorders (7.6\%). Among the 114 survey respondents to date, $90.4 \%$ reported that they had referred patients to navigation, most commonly for mood $(97.1 \%)$ and anxiety $(86.4 \%)$ disorders. PCPs indicated that they made referrals because they wanted help with finding $\mathrm{BH}$ providers within their patients' insurance networks $(92.2 \%)$, ensuring that patients received timely $\mathrm{BH}$ care $(75.7 \%)$, and determining the appropriate type of $\mathrm{BH}$ provider or level of care $(41.8 \%)$. Of the PCPs surveyed, $82.5 \%$ indicated that they would like the navigation program to continue after the grant-funded quality improvement project ends; $72.8 \%$ agreed that navigation enhanced clinical care; $68.9 \%$ believed that navigation improved patients' care experience; and $60.2 \%$ reported that navigation increased their own confidence about addressing patients' $\mathrm{BH}$ needs. However, only $58.3 \%$ of PCPs positively rated their overall experience with the navigation program; $36.9 \%$ reported they had not made referrals in the past 3 months, in part because their past referrals had not been processed in a timely manner $(36.8 \%)$.

Conclusion: PCPs appear to value the services provided by the navigation program and would like for it to continue despite the challenges associated with high demand and limited resources. This highlights the potential utility of $\mathrm{BH}$ navigation as an approach to improving the health care of teens and for enhancing the practices of those physicians who care for them. Keywords: child and adolescent health, program evaluation, research, primary care, quality improvement, behavioral and mental health, dissemination, implementation, innovation 
P5.04

Changes in Primary Care Physicians' Perspectives on Adolescent Behavioral Health Within the Context of a Quality Improvement Project

Jinnan Li, ${ }^{1}$ Ellis Dillon, ${ }^{1}$ Martina Li,${ }^{1}$ Kimberly Erlich, ${ }^{2}$ Amy Heneghan, ${ }^{2}$ Yan Yang, ${ }^{1}$ Ming Tai-Seale,${ }^{2,3}$ Daniel Becker $^{2}$

${ }^{1}$ Palo Alto Medical Foundation Research Institute, Mountain View, CA; ${ }^{2}$ Palo Alto Medical Foundation, San Mateo, CA; ${ }^{3}$ University of California, San Diego, San Diego, CA

Background: Approximately $20 \%$ of U.S. adolescents have a behavioral health $(\mathrm{BH})$ condition. Early detection and effective treatment can ease their burden. The Adolescent Behavioral Health Project is a multifaceted, integrated $\mathrm{BH}$ quality improvement effort, implemented over a 4-year period within a multispecialty medical practice. This study examines the extent to which primary care physicians' (PCPs) knowledge and views on teen $\mathrm{BH}$ changed during this implementation period.

Methods: All pediatric and family medicine PCPs were invited to complete a survey, which included questions from American Academy of Pediatrics Periodic Survey and the Physician Belief Scale, during 2013-2014 (baseline) and in 2017 (follow-up).

Results: Survey response rate was $49.3 \%$ at baseline and $51.6 \%$ at follow-up. Compared to $21.4 \%$ at baseline, $62.2 \%$ of PCPs stated that they believe mental health conditions are extremely/very common in their teen patients in the followup survey; $17.1 \%$ stated that substance abuse is extremely/ very common in the follow-up survey, compared to $2.6 \%$ at baseline. PCPs reported higher levels of confidence in the follow-up survey in their abilities to identify teen $\mathrm{BH}$ problems (baseline: $76.9 \%$; follow-up: $88.3 \%$ ) and to make effective referrals (baseline: 51.3\%; follow-up: 67.6\%). Throughout the project period, a relatively small proportion of PCPs reported feeling confident in their abilities to provide brief counseling (baseline: 37.6\%; follow-up: $37.8 \%$ ) and psychopharmacotherapy (baseline: $27.4 \%$; follow-up: $28.8 \%$ ). Nonetheless, the majority agreed that teens want their PCPs to treat their BH problems (baseline: 76.1\%; follow-up: 70.3\%). Time constraints appeared to be a persistent concern for PCPs, with the majority continuing to report that time does not allow them to consider $\mathrm{BH}$ problems during visits (baseline: 52.1\%; follow-up: $60.4 \%$ ), that evaluating or treating $\mathrm{BH}$ problems would cause them to become overburdened (baseline: $58.1 \%$; follow-up: $64.0 \%$ ) and that investigating $\mathrm{BH}$ problems will decrease their work efficiency (baseline: $63.25 \%$; followup: $68.5 \%$ ).

Conclusion: Approximately 3 years after implementation of an integrated BH project, PCPs reported improved awareness of $\mathrm{BH}$ problems and greater confidence in their abilities to identify $\mathrm{BH}$ needs and to make effective referrals. However, substantial barriers remain that may deter PCPs from addressing teen $\mathrm{BH}$ problems and highlight the need for additional educational and systemlevel interventions.

Keywords: child and adolescent health, survey research and methods, primary care, behavioral and mental health

\section{P5.05}

\section{Identifying Asthma Severity and Visits for Asthma Exacerbations From Chart Audit Data}

\author{
James D. Nordin, ${ }^{1}$ Gabriela Vazquez-Benitez, ${ }^{1}$ Elyse \\ O. Kharbanda, ${ }^{1}$ Avalow Olsen, ${ }^{1}$ Leslie Kuckler ${ }^{1}$ \\ ${ }^{1}$ HealthPartners Institute, Minneapolis, $M N$
}

Background: Asthma is the most common chronic medical condition in children. Prior observational studies of influenza vaccine safety in asthmatic children have been limited due to confounding by indication. Improved methods are needed to classify asthma severity and to identify visits for asthma exacerbations from automated data. These data represent the first step in that process.

Methods: We reviewed charts of a random sample of 100 subjects, age 2 to 18 years, with clinic visits between 2011 and 2016. All visits with an asthma or wheezing diagnosis in 42 days following influenza immunization were classified as management or exacerbation using a chart algorithm based on positive physical findings or in-clinic treatments for asthma. Visit assessment and plan were available. Children were classified as having asthma controlled and whether asthma was persistent or intermittent using visits occurring from 12 months prior to the day of influenza immunization. Asthma control was classified based on asthma control test (ACT) scores of $\geq 20$ (controlled) or $<20$ (uncontrolled). Persistent or intermittent was determined based on classification in asthma management plans (AMPs) and prescription of controller medications.

Results: A total of 77 office visits with a diagnosis of asthma or wheezing were available. The chart audit algorithm was compared to the chart assessment and plan (kappa $=0.67$ ). Of the 93 subjects with complete chart audits, based on ACT, 45 subjects were classified as controlled, 18 were classified as not controlled, and 30 had no ACTs. In AMPs, 21 (38\%) were classified as persistent, 34 were intermittent, and 38 had no AMP classification. Based on medication prescriptions only, $75(80 \%)$ were persistent and 18 were intermittent.

Conclusion: An algorithm based on chart audit findings was moderately accurate in determining which visits were for management and which were for exacerbation. AMPs classified far fewer patients as persistent compared to controller medications. All of these determinations will be compared to electronic data algorithms to see which match best. The final electronic data algorithms will be used to analyze the entire cohort.

Keywords: child and adolescent health, chronic disease 
P5.06

Costs of Clinical Decision Support on the Recognition and Management of Hypertension in Youth

Steven P. Dehmer, ${ }^{1}$ Nicole K. Trower, ${ }^{1}$ Stephen E. Asche,${ }^{1}$ Heidi L. Ekstrom, ${ }^{1}$ James D. Nordin, ${ }^{1}$ Patrick J. O'Connor, ${ }^{1}$ Alan R. Sinaiko, ${ }^{2}$ Elyse O. Kharbanda ${ }^{1}$

${ }^{1}$ HealthPartners Institute, Minneapolis, $M N ;{ }^{2}$ University of Minnesota, Minneapolis, MN

Background: Blood pressure (BP) is routinely measured in youth, but hypertension (HTN) is often not clinically recognized. This study evaluates the economic outcomes of a randomized clinical trial (TeenBP) of an electronic health record-embedded clinical decision support (CDS) tool designed to assist in the recognition and management of hypertension in youth.

Methods: Twenty primary care clinics within an integrated health system were randomized to the TeenBP CDS or usual care from April 15, 2014, to April 14, 2016. Differences in primary care costs between study arms, defined by CPT-4 codes for new or existing patients seen in a clinic setting, were compared in the 12 months pre- and post-study enrollment using total paid insurance claim amounts. Continuous insurance coverage, administered by an affiliated health plan, was required over the comparison period for inclusion. Three comparison groups were defined by patients meeting the following criteria: 1 ) at least 2 visits within 1 year with $\mathrm{BP} \geq 120 / 80 \mathrm{mmHg}$ or $\mathrm{BP} \geq 90$ th and $<95$ th percentile (ie, pre-HTN); 2) 1 visit with $\mathrm{BP} \geq 95$ th percentile (ie, isolated elevated BP); and 3 ) at least 3 visits within 1 year with $\mathrm{BP} \geq$ 95th percentile (ie, HTN). Generalized estimating equation models, gamma-distributed with a log link, were used to estimate differences between groups over time.

Results: Of the 4990,3173, and 472 patients meeting criteria for pre-HTN, isolated elevated BP, and HTN, respectively, 1520,851 , and 153 also met the continuous insurance coverage requirement for inclusion in the analysis. Relative to patients in usual care clinics, the pre-to-post difference in primary care costs for patients in TeenBP CDS clinics were lower: $-\$ 36$ for pre-HTN $(\mathrm{P}=0.10),-\$ 12$ for isolated elevated BP $(\mathrm{P}=0.65)$, and $-\$ 117$ for HTN $(\mathrm{P}=0.13)$. None of these differences were statistically significant at the $95 \%$ confidence level.

Conclusion: This analysis indicates that embedding a CDS tool to improve the recognition and management of hypertension in youth did not lead to increases in costs associated with primary care utilization. Further investigation is needed to determine effects on other medical costs, including laboratory services, specialty care, and pharmacy.

Keywords: child and adolescent health, economic studies
P5.07

Development of a Clinical Decision Support System for Pediatric Abdominal Pain in Emergency Room Settings

Heidi L. Ekstrom, ${ }^{1}$ Elyse O. Kharbanda, ${ }^{1}$ Gabriela Vazquez-Benitez, ${ }^{1}$ Steven P. Dehmer, ${ }^{1}$ Patrick J. O'Connor, ${ }^{1}$ Gopi Kunisetty, ${ }^{1}$ Rashmi Sharma, ${ }^{1}$ Anupam Kharbanda ${ }^{2}$

${ }^{1}$ HealthPartners, Bloomington, MN; ${ }^{2}$ Children's Hospitals of Minnesota, Minneapolis, MN

Background: Appendicitis is a common surgical emergency in children, yet the diagnosis can be challenging. An electronic health record (EHR)-based clinical decision support system (CDSS) designed to help guide management of pediatric patients with acute abdominal pain was implemented as part of a multisite pragmatic cluster-randomized controlled trial. Methods: The CDSS was designed for rapid two-way communication between the EHR and a web service that contains clinical algorithms and a validated risk calculator. The tool displays risk for appendicitis and management suggestions. The CDSS was developed and implemented in two phases. Phase 1 consisted of a best practice alert (BPA) with a link to three questions to identify study eligibility for the nurse or treating provider to complete. The provider also has the option to choose an acknowledgment reason via the BPA if appendicitis is not suspected. Phase 2 consisted of another BPA directed toward the provider with a link to the CDSS tool. The tool was designed for ease of use and to accommodate differences in workflow. Weekly response rate reports are circulated to all sites, and nurses who complete the questions are entered into a drawing for a chance to win a $\$ 50$ gift card.

Results: Accommodating variations in workflow, staffing, and competing priorities in a busy emergency setting has been challenging. Minor edits to the tool were ongoing during Phase 1 in response to user feedback. Adjustments also occurred for a short period during Phase 2. Training and outreach occurred throughout the project. Response rates at the smaller sites consistently ranged from $77 \%$ to $93 \%$. Response rates at the larger urban sites were $61 \%-70 \%$.

Conclusion: Implementation was challenging and uptake of the tool has been highly dependent on local workflow practices as well as urban versus rural setting. Addressing variations in workflow and gaining trust of providers to use the tool has been an ongoing challenge. In general, smaller rural sites have had better uptake and less workflow variation than larger urban sites. More qualitative feedback from providers and nurses is needed to help identify and mitigate barriers to use.

Keywords: child and adolescent health, acute inpatient care, pragmatic trials, clinical decision-making, dissemination, implementation, innovation 


\section{DATA SCIENCE, INFORMATICS, DATA MODELS}

\section{P6.01 \\ Our Divided World: Can We Predict Division Affiliation for the Unaffiliated?}

\section{Roy Pardee ${ }^{1}$}

${ }^{1}$ Kaiser Permanente Washington, Seattle, WA

Background: Kaiser Permanente Washington (KPWA) members fall into one of two divisions - group practice (GPD) or contracted network. Division controls the degree to which their care is provided at KPWA clinics by members of our physician group. The distinction has important implications for capture of clinical (as opposed to just billing) data and is a crucial component of our implementation of the completeness-of-capture variables. A change in upstream enrollment data significantly improved our ability to make these distinctions, at the cost of a $12 \%-15 \%$ missing rate (generally people without any utilization). That rate is unacceptably high and so we set out to devise and evaluate an imputation method for classifying these nonutilizers into divisions.

Methods: Imputation was done using just two variables, insurance product category and county of residence, which are reliably populated for all. Crossing those two variables yields about 420 different cells in a given month. For each cell we calculate a probability that an unaffiliated person will ultimately affiliate with the GPD by taking the proportion of affiliated people in that cell that are definitively GPD-affiliated. So if there are 120 people in a cell, 100 of whom are affiliated one way or another and 80 of those are GPD-affiliated, we assign a probability of 0.8 to the 20 unknowns.

Results: To evaluate these imputations we isolated 107,775 instances in which a member went from an imputed value to a definitive value and used the former as the sole predictor in a logistic regression predicting the latter. Predictive power was quite good, giving an overall area under curve of 0.88 . Inspection of sensitivity and specificity rates at different cut-points revealed that 0.8 is a good criterion to use for applications for which we need to divide members cleanly into the two groups.

Conclusion: Using a simple imputation method, we were able to triangulate from known data (proportion in group affiliating with GPD) to unknown information (probable future affiliation) in a highly accurate and useful manner. We can safely use the imputed values to set enrollment flags like incomplete_emr and incomplete_lab.

Keywords: managed care (features), virtual data warehouse, health care organization
P6.02

The Vaccine Safety Datalink Pregnancy Episode Algorithm: Updates, Enhancements, and Validation Work

Stephanie Irving, ${ }^{1}$ Don Bachman, ${ }^{1}$ Brad Crane, ${ }^{1}$ Kimberly Vesco, ${ }^{1}$ Stacy Harsh, ${ }^{1}$ Sanchita Sengupta, ${ }^{1}$ Elyse O. Kharbanda, ${ }^{2}$ Gabriela Vazquez-Benitez, ${ }^{2}$ Natalie McCarthy, ${ }^{3}$ Allison Naleway ${ }^{1}$

${ }^{1}$ Kaiser Permanente Center for Health Research, Portland, OR; ${ }^{2}$ HealthPartners Institute, Minneapolis, $M N ;{ }^{3}$ Centers for Disease Control and Prevention, Atlanta, GA

Background: The Vaccine Safety Datalink (VSD) is a collaboration between the Centers for Disease Control and Prevention and 7 integrated health care delivery systems. Evaluating the safety of vaccination during pregnancy is an important objective of the VSD, and accurate identification of pregnancies, pregnancy start and end dates, and pregnancy outcomes is vital to VSD research. The VSD previously developed an algorithm to retrospectively identify pregnancies using electronic medical record, claims, and birth certificate data. Modifications to the original VSD pregnancy episode algorithm (PEA) are underway to update the algorithm with ICD-10 codes and identify additional sources of gestational age data.

Methods: To update the PEA to accommodate the shift from ICD-9 to ICD-10 codes, we reviewed and modified the diagnosis and procedure code lists used to identify potential pregnancies. In partnership with the Kaiser Permanente Center for Safety and Effectiveness Research pregnancy working group, we identified and incorporated several new gestational age-specific ICD-10 codes. To validate these modifications to the PEA, manual review of pregnancy dates and outcome types will take place at all VSD sites.

Results: ICD-10 codes were added to the PEA diagnosis and procedure lists in early 2017 . The PEA's SAS program was then modified to utilize ICD-10 codes and incorporate the information from gestational age-specific ICD-10 codes into the estimate of pregnancy onset. The modified PEA was pilot tested in May 2017 with data from all VSD sites; 153,080 pregnancies were identified in 2015. Live births and spontaneous abortions accounted for $66 \%-79 \%$ and $13 \%-15 \%$ of all pregnancy outcomes, respectively, by VSD site. Preliminary data indicate that the proportions of live births were similar when restricted to ICD-9 or ICD-10 coding eras and that the updates to the algorithm may have reduced misclassification of spontaneous abortions. The modified PEA will be run on updated VSD data to identify pregnancies through 2016; validation of these pregnancies will begin in November 2017.

Conclusion: The VSD PEA is an important tool for studies of vaccine safety during pregnancy. Recent modifications have accommodated the transition to ICD-10 coding and 
refined capture of gestational age. Future work will focus on real-time identification of pregnancies and preconception care in the VSD population.

Keywords: observational study, technology assessment, epidemiology

\section{P6.03}

Development of a Research Network for Biosimilars: Creation and Operation of the BBCIC

\author{
Pamala A. Pawloski, ${ }^{1}$ Kevin Haynes, ${ }^{2}$ Daniel Kent, ${ }^{3}$ \\ Cheryl McMahill-Walraven, ${ }^{4}$ Catherine Panozzo, ${ }^{5}$ \\ Vanita Pindolia, ${ }^{6}$ Jeffrey Brown, ${ }^{5}$ Charles Barr, ${ }^{7}$ \\ Bernadette Eichelberger ${ }^{8}$
}

${ }^{1}$ HealthPartners Institute, Bloomington, MN; ${ }^{2}$ Anthem HealthCore, Wilmington, DE; ${ }^{3}$ Kaiser Permanente Washington, Seattle, WA; ${ }^{4}$ Aetna, Blue Bell, PA; ${ }^{5}$ Harvard Pilgrim Health Care Institute, Boston, MA; ${ }^{6}$ Henry Ford Health System, Detroit, MI; ${ }^{7}$ Academy of Managed Care Pharmacy, Alexandria, VA; ${ }^{8}$ Biologics and Biosimilars Collective Intelligence Consortium, Alexandria, VA

Background: The Biologics and Biosimilars Collective Intelligence Consortium (BBCIC) initiated research in third quarter of 2016 to generate postmarket evidence in anticipation of the entrance of biosimilars to the U.S. market. The BBCIC's scientific approach was conducted to answer patient and physician concerns about biosimilars and to potentially facilitate greater acceptance and adoption of biosimilars.

Methods: The BBCIC network leverages a Sentinel distributed research network (DRN). The BBCIC collaboration consists of 18 member organizations, including managed care, integrated delivery networks, pharmacy benefit managers, industry, and researchers. In preparation for the introduction of biosimilars in the marketplace, four descriptive analysis protocols were conducted to characterize BBCIC DRN data - including the identification of demographic and clinical patterns that determine differences in utilization and outcomes. The descriptive analysis projects and their primary outcome measures: 1) first-cycle granulocyte colony-stimulating factor (G-CSF) prophylaxis for breast and lung cancer (outcome: hospitalizations for febrile neutropenia); 2) erythropoietin-stimulating agent (ESA) use in hemodialysis; 3) long-acting insulin use in types 1 and 2 diabetes (outcomes: major adverse cardiac events and hypoglycemia; and 4) anti-inflammatory agent use for rheumatoid arthritis and psoriatic and gastrointestinal conditions (outcome: hospitalizations for serious infections). Results: Preliminary descriptive analysis results demonstrated: 1) We can identify G-CSF prophylaxis vs treatment use, full vs partial treatment duration, and rare safety events; 2) For upcoming comparative effectiveness research (CER), the BBCIC will use Medicare's end-stage renal disease data set as its ESA data are more granular than BBCIC DRN data; 3) For long-acting insulin, primary outcomes are comparable to other observational trials; and 4) For anti-inflammatory agents, serious infection outcomes are comparable to other observational trials.

Conclusion: The BBCIC demonstrated outcomes comparable to those in the published literature. Following accumulation of sufficient biosimilar exposures, CER will be initiated to assess the safety and effectiveness of individual biosimilars compared with innovator compounds. In preparation, work groups have been convened to define best practices for the conduct of switching pattern and CER studies; for transitioning from International Classification of Diseases, Ninth Revision, Clinical Modification (ICD-9-CD) to ICD10-CM mapping; and for monitoring data partner capture of National Drug Code and Healthcare Common Procedure Coding System codes for biosimilar injectables.

Keywords: pharmacy, observational study, epidemiology, pharmaceutical prescribing/use

\section{P6.04}

\section{Mining Time-Stamped Electronic Health Record Data to Understand Physician Work Patterns}

\author{
Dorothy Hung, ${ }^{1}$ Qianyun Zhang, ${ }^{1}$ Quan Truong, ${ }^{1}$ Su- \\ Ying Liang, ${ }^{1}$ Harold S. Luft ${ }^{1}$
}

${ }^{1}$ Palo Alto Medical Foundation Research Institute, Mountain View, $C A$

Background: As primary care continues to face high patient demand and provider burnout, interventions to improve efficiency first require understanding physician work patterns. We leveraged time-stamped electronic health record (EHR) data to reconstruct daily physician time working. This was achieved by processing a sample of 15 million daily transactions among 303 physicians over 4 years.

Methods: EHR access logs were used to quantify daily physician work activities. We focused on activities occurring before, during, and after patient visits and noted work locations (examination room, physician's desk, remotely). We addressed challenges in accurately identifying start/ end of patient care due to patients arriving early or late or physicians running ahead or behind schedule. Another complication was EHR capture of exam room "batching," which is when a physician is charting in an empty exam room, creating false indications of a patient visit.

Results: We addressed these challenges using iterative data mining and validation techniques. "Start of Patient Care" time-points were identified by selecting the first EHR transaction in an exam room with a patient, following that patient's check-in time. "End of Patient Care" was identified by the last EHR exam room transaction for the last arriving patient within 2 hours of the check-in time. This strategy identified end points for $88.2 \%$ of physician workdays. For the remaining $11.8 \%$, we selected the last transaction in an exam room occurring $<2$ hours of a patient's check-in time, 
or alternatively, $<2$ hours of the appointment time (if the patient checked in early). Using these approaches, we found that physicians spent an average of 36 minutes working before the start of patient care each day, and 1 hour and 16 minutes after the last visit of the day. Among physicians who accessed the EHR remotely, approximately 1 hour and 7 minutes were spent working from home daily. Overall, physicians spent about 8 hours and 53 minutes working per day, including activities at the desk, during patient appointments, and outside the office.

Conclusion: This study augments traditional, time-motion research by using time-stamped EHR data to reconstruct physician workdays over several years. Our approach to describing physician work time may inform approaches used by other health systems seeking to improve efficiency.

Keywords: information technology, primary care

\section{P6.05}

Tumor Registry Information Extraction Tool (TRInE-T): A Supplemental Abstraction Tool for Tumor Registrars

\section{Celeena Jefferson, ${ }^{1}$ Aung Oakkar, ${ }^{1}$ Alphonse Derus, ${ }^{1}$ Jackie Blank, ${ }^{1}$ Monica Ter-Minassian ${ }^{1}$}

${ }^{1}$ Mid-Atlantic Permanente Research Institute, Kaiser Permanente Mid-Atlantic States, Rockville, MD

Background: Tumor registrars at Kaiser Permanente MidAtlantic States (KPMAS) are responsible for identifying all incidences of cancer among members and abstracting malignancy details into CNExT (tumor registry software). Abstraction for tumor registry compliance is a time-consuming task that requires searching multiple modules within the electronic medical record (EMR). At KPMAS, the tumor registry team developed the Tumor Registry Information Extraction Tool (TRInE-T) to supplement the abstraction process and minimize the time required to complete an abstract. Methods: To develop TRInE-T, we created SAS programs to extract structured data elements weekly from federated sources, including the EMR. Tumor-related encounters were identified using CPT and ICD (9th/10th revision) codes, and the Cancer Research Network chemotherapy lookup tables were utilized to capture additional disease and treatment information. We followed the Facility Oncology Registry Data Standards guidelines and used CNExT's tumor abstract template as a model for content, layout, and workflow. The resulting data sets for TRInE-T consisted of encounter information (including dates for diagnoses, procedures, and cancer treatment), social history, demographics (current and historic), and pathology reports. A SAS stored process was created to provide users access to the data on the SAS Portal website. When users log on to the site, they are prompted to enter a medical record number. If cancer encounters exist for the patient, TRInE-T returns HTML output of the data onto one page and in the order of the CNExT tumor abstract template; if no cancer encounters exist, no data is displayed. To ensure data security, access provision for TRInE-T is strictly limited and all medical record number searches are stored in an audit log.

Results: During user-acceptance testing, tumor registrars reported quicker identification of patient information, especially treatment dates. Immediate access to this information reduced the time required to search the EMR while abstracting.

Conclusion: TRInE-T is a data organizational tool that provides a quick overview of structured patient information, supplementing tumor registry abstraction and limiting the need to search the EMR extensively. With increased use of the tool, audit logs can serve as the foundation for application metrics. This tool could be adapted for other tumor registries within the Health Care Systems Research Network.

Keywords: cancer, information technology

P6.06

Precision and Census Block Group Estimates

\section{Christopher Mack ${ }^{1}$}

${ }^{1}$ Kaiser Permanente Washington Health Research Institute, Seattle, WA

Background: Researchers want the most specific data from the most granular geographies to estimate the social and economic characteristics of the neighborhoods of their populations. The American Community Survey (ACS) releases 5-year combined estimates at the block group level for the majority of block groups in the United States. This data would seem to be the most specific and most granular. But how reliable are those estimates?

Methods: The variation of block group estimates within census tracts can be broken into three components: observed variation across point estimates, margins of error calculated by the ACS, and true error. The last cannot be measured, but using U.S. Census data we can distinguish between the amount of variance driven by observed differences is point estimates and the amount of variance driven by sampling error.

Results: In preliminary analysis, we found 30\%-50\% of variation in block group estimates to be driven by sampling error, rather than by the observed differences in point estimates.

Conclusion: If the variance from sampling error approaches or exceeds that of the observed differences, we would have a hard time believing that the available point estimates reflect actual differences. For this reason, we would guide users to resist the temptation to use the misleadingly attractive block group data and instead use the more reliable data available at the census tract level, despite the lack of granularity.

Keywords: geographic information systems, spatial factors, demographics, virtual data warehouse, social determinants of health 
P6.07

\section{Common Entities Among Common Data Models}

\section{John Weeks ${ }^{1}$}

${ }^{1}$ Kaiser Permanente Washington Health Research Institute, Seattle, WA

Background: The value of having a health care common data model for collaborative research has been gaining recognition since the virtual data warehouse (VDW) emerged in 2003. In the last 15 years many different common data models have been introduced for different purposes. This poster presents a visual interpretation of the history and shared entities across many different popular models.

Methods: Helping people to understand the history of common data models can help to bring awareness of the shared work between these efforts. It also can help researchers understand which models might best fit the needs of their study. As we continue to build bridges between researchers using shared data, an understanding of the history of the VDW and the models that followed it is essential.

Results: The different technologies that are used to inform the technology and some of the advantages and disadvantages will be touched on as a part of this poster. Having a basic understanding of some of the computer science concepts that inform different technological decisions also can help researchers in understanding the model that they are working with.

Conclusion: Finally, the necessity of testing and its value to the research community will be addressed by comparing the two most common approaches to testing. The poster will show different models' approaches to quality assurance and the advantage of data curation to research and operational concerns in a learning health care setting.

Keywords: information technology, virtual data warehouse, research administration, contracting, operations

\section{P6.08}

The Vaccine Safety Datalink and Immunization Information Systems (IIS): Evaluating the Contribution of IIS Data

Holly Groom, ${ }^{1}$ Bradley Crane, ${ }^{1}$ Stephanie Irving, ${ }^{1}$ Natalie McCarthy, ${ }^{2}$ Matthew Daley, ${ }^{3}$ James Donahue, ${ }^{4}$ Sungching Glenn, ${ }^{5}$ Kristin Goddard, ${ }^{6}$ Michael Jackson, ${ }^{7}$ Erika Kiniry, ${ }^{7}$ Ned Lewis, ${ }^{6}$ Marlene Lugg, ${ }^{5}$ Lawrence Madziwa, ${ }^{7}$ Erica Scotty, ${ }^{4}$ Lina Sy, ${ }^{5}$ Kris Wain, ${ }^{3}$ Allison Naleway ${ }^{1}$

${ }^{1}$ Kaiser Permanente Northwest, Portland, OR; ${ }^{2}$ Centers for Disease Control and Prevention, Atlanta, GA; ${ }^{3}$ Kaiser Permanente Colorado, Denver, CO; ${ }^{4}$ Marshfield Clinic, Marshfield, WI; '5aiser Permanente Southern California, Pasadena, CA; ${ }^{6}$ Kaiser Permanente Northern California, Oakland, CA; ${ }^{7}$ Kaiser Permanente Washington, Seattle, WA
Background: Using data from 7 integrated health care systems (sites), the Vaccine Safety Datalink (VSD) evaluates vaccine safety across the lifespan. All VSD sites use electronic health record (EHR) data to determine the vaccination status of their members; 5 sites complement the EHR with data from their state immunization information systems (IIS). IIS are confidential, population-based databases administered by state health departments that contain a consolidated record of vaccine doses administered within a defined geographic area. Our goal was to assess the contribution of IIS data to the vaccination records of members at each VSD site and to determine whether that contribution differs by member demographics (ie, age, enrollment duration) or by vaccine type.

Methods: We used administrative health care databases at 4 participating VSD sites to quantify the respective contribution of IIS and EHR data to their members' vaccination records from 2000 through 2015. The assessments included: 1) total doses administered, by age at assessment $(5,12$, and 45 years) and enrollment duration; and 2) vaccine coverage estimates for diphtheria-tetanus-acellular pertussis (DTaP) and human papillomavirus (HPV) vaccines, with and without inclusion of IIS data.

Results: Shorter duration of health care system enrollment was associated with higher contributions from IIS data among all age groups. Depending on age of assessment $(5,12$, or 45 years), IIS contributed $5 \%-40 \%$ of total doses administered for members with $\leq 3$ years of continuous enrollment. Including IIS data increased DTaP coverage estimates by $20 \%-35 \%$ among children who had $\leq 2$ years of continuous enrollment for 3 of the 4 sites; the fourth site observed no added benefit from IIS data for its pediatric population. Including IIS data increased HPV coverage estimates for 15-26-year-olds by $4 \%-10 \%$. This was evident across the 4 sites for members with $\leq 7$ years of continuous enrollment.

Conclusion: IIS appear to contribute a measurable amount of vaccine data that would not otherwise be available to the health care system. Enrollment duration and vaccine type impact the proportionate contribution of IIS data. Including IIS data improves completeness for studies pertaining to vaccination.

Keywords: virtual data warehouse, epidemiology

P6.09

Building the eMERGE Observational Medical Outcomes Partnership Common Data Model Using the Virtual Data Warehouse

John Weeks, ${ }^{1}$ David Carrell ${ }^{1}$

${ }^{1}$ Kaiser Permanente Washington Health Research Institute, Seattle, WA

Background: The Electronic Medical Records and Genomics Network (eMERGE) was formed "to develop, disseminate, and apply approaches to research that combine 
biorepositories with electronic medical record (EMR) systems for genomic discovery and genomic medicine implementation research" (https://emerge.mc.vanderbilt. edu/about-emerge/). As part of this mission, eMERGE has decided to implement Observational Health Data Sciences and Informatics' Observational Medical Outcomes Partnership (OMOP) common data model (CDM) to simplify sharing of phenotype definitions (eg, operational definitions of patients with/without particular diseases) across sites.

Methods: To expedite this process, we have decided to leverage our virtual data warehouse implementation to populate eMERGE's OMOP CDM. The high-level process of mapping between the two models will be illustrated. We also will add details of some of the challenges in bridging the two models. Core entities used to provide phenotype data also will be illustrated.

Results: We will share the results of the project and share some of the challenges discovered as part of the implementation.

Conclusion: As genetic information continues to be of interest for research, finding ways to share this information will continue to be an important subject area for common data models to consider in their implementation.

Keywords: genetics, genomics, information technology, virtual data warehouse

P6.10

Cervical Pathology Data Capture in a Mixed-Model Health Care Delivery System

Aruna Kamineni, ${ }^{1}$ Gabrielle Gundersen, ${ }^{1}$ Malia Oliver, ${ }^{1}$ Madziwa Lawrence, ${ }^{1}$ Vina Graham ${ }^{1}$

${ }^{1}$ Kaiser Permanente Washington Health Research Institute, Seattle, WA

Background: Evaluating cervical cancer screening for population health management requires outcomes data which, in most health care settings, must be extracted from pathology reports containing unstructured text. In a mixedmodel health care delivery system that contracts pathology services, capture of these data may pose additional challenges. We describe a cervical pathology data capture strategy for a cohort of 18-89-year-old women enrolled during 20102014 at Kaiser Permanente Washington (KPWA), a health care system with both internal and contracted pathology services.

Methods: KPWA pathology reports are available in a database, and those from contracted providers may be available as transcriptions or scanned reports in the electronic health record (EHR). We identified all KPWA reports and external transcriptions for the cohort, loaded only records containing cervix-specific terms into an ACCESS database, and abstracted them for cervical precancers and cancers. We identified all claims for cervical diagnostic and treatment procedures on the cohort and linked them to abstracted pathology records. As gynecological surgery can have multiple indications, we identified surgical procedures with diagnosis codes indicating cervical intraepithelial neoplasia grade 3 or worse to capture those that were performed for cervical treatment. Unlinked abstracted pathology records were set aside, and unlinked claims records that were not procedures without pathologic specimens (ie, ablations [ $n=171]$ and colposcopies without biopsies $[n=1941])$ or surgeries with noncervical indications were assumed to represent scanned pathology reports. We reviewed the EHR to locate these reports and abstract outcomes.

Results: We identified 14,858 cervical pathology records for our cohort; 7734 (52.0\%) KPWA reports and 460 (3.1\%) transcriptions linked to cervical procedures from claims. We abstracted 620 (4.2\%) scanned reports, but 976 (6.6\%) external pathology reports from cervical procedures were missing in the EHR. Remaining pathology records $(\mathrm{n}=5070$ [34.1\%]) represented gynecologic surgeries for noncervical indications, noncervical procedures with cervical sampling (eg, endometrial biopsy with endocervical curettage), or coding errors in claims data.

Conclusion: Although data may not be complete, efficient capture of cervical pathology outcomes in a mixed-model health care setting can be achieved by aggregating all available data sources and first addressing electronically available text records to identify and minimize resourceintensive EHR review of scanned reports.

Keywords: cancer, quality improvement

\section{DIABETES, OBESITY, OTHER CHRONIC CONDITIONS}

\section{P7.01 \\ Determinants of Treatment Intensification Among Patients With Type 2 Diabetes in U.S. Clinical Practice: A Systematic Review}

Nina Szwerinski, ${ }^{1}$ Catherine Nasrallah, ${ }^{1}$ Vidita Chopra, ${ }^{1}$ Linda Lee, ${ }^{2}$ Robert Romanelli ${ }^{1}$

${ }^{1}$ Palo Alto Medical Foundation, Palo Alto, CA; ${ }^{2}$ University of California, San Francisco, San Francisco, CA

Background: Delayed treatment intensification is a main contributor to suboptimal glycemic control in patients with type 2 diabetes (T2D). An understanding of factors that contribute to treatment intensification is required to design novel health care interventions to mitigate clinical inertia and improve patient outcomes. We sought to synthesize the extant literature on factors that contribute to treatment intensification with second-line therapy among patients with T2D in real-word clinical practice in the United States.

Methods: We systematically searched PubMed, Embase, 
and Web of Science between January 1, 2000, and August 30, 2017, for research articles on treatment intensification in T2D. We included peer-reviewed observational studies in English, conducted on U.S. adults. We included studies of treatment intensification determinants using quantitative, qualitative, or mixed-method analyses. We excluded randomized controlled trials and studies conducted in inpatient settings.

Results: We identified 20,493 unique citations. In a preliminary analysis, we found 8 health care system electronic health record (EHR)-based studies that examined quantifiable patient-level factors as contributing to treatment intensification with second-line T2D therapy. Six of the 8 studies were conducted in managed-care health care systems. Consistently, younger age, higher hemoglobin A1c levels, and better adherence to first-line therapy were associated with having treatment intensified, which was confirmed by at least 3 qualitative studies. Two health care system EHRbased studies examined quantifiable health care providerlevel factors in treatment intensification. One study, conducted in a multipayer system, found that younger health care providers and specialty vs primary care providers were more likely to intensify treatment. Another study from a managed care organization found that provides' age, gender, panel size, and type were not associated with intensification. No studies included in this review examined health care system-level factors. An updated analysis will be presented. Conclusion: In real-world clinical practice, our preliminary findings suggest that treatment intensification is influenced by patients' age, glycemic levels, and past medication adherence. Information on the role of health care providerand system-level factors is scarce. More studies are needed to understand the factors that contribute to treatment intensification, particularly at the provider and system level as well as in multipayer settings, in order to design appropriate $\mathrm{T} 2 \mathrm{D}$ health care interventions.

Keywords: systematic review, meta-analysis, ambulatory/ outpatient care, diabetes, chronic disease

\section{P7.02}

The Prevalence of Overweight and Obesity in Communities Engaging in Interventions Aimed at Preventing Childhood Obesity

Gabriela Vazquez-Benitez, ${ }^{1}$ Marna Canterbury, ${ }^{2}$ Julia Johnson, ${ }^{2}$ Andrea Chinchilla Elizondo, ${ }^{3}$ Juliana Tillema, ${ }^{3}$ Thomas Kottke, ${ }^{1}$ Elsa Keeler ${ }^{4}$

${ }^{1}$ HealthPartners Institute, Minneapolis, MN; ${ }^{2}$ Lakeview Hospital, Stillwater, MN; ${ }^{3}$ HealthPartners, Minneapolis, MN; ${ }^{4}$ HealthPartners Medical Group, White Bear Lake, MN

Background: Through PowerUp and BearPower, HealthPartners supports community-level interventions to make better eating and physical activity "easy, fun and popular" for school-aged children and their families in the St. Croix River Valley area. The interventions focus on community engagement to create multilevel community change. Goals include changes in the food and physical activity environment, community partnerships and outreach, and programming in school and clinical settings. We present preliminary child overweight and obesity data in intervention communities and surrounding reference communities.

Methods: We collected data on weight and height for children age 6 to 11 years from the HealthPartners clinical warehouse. We selected measurements from well-child visits when available, otherwise we used data from the most recent clinic visit. We defined obesity as a body mass index $(B M I) \geq 95$ th percentile of the Centers for Disease Control and Prevention's sex-specific BMI-for-age growth charts. We defined overweight as BMI $\geq 85$ th and $<95$ th percentile. We classified children as living in school districts where the intervention took place (Valley and White Bear Lake areas) or not (other). Initiation of the intervention was staggered from 2013 to 2015 . We collected data on age, gender, race/ethnicity, payer, and care group. We compared trends in the unadjusted prevalence of overweight or obesity from 2011-2012 to 2015-2016 between intervention and reference communities. Results: A total of 8710 children had a home address in the intervention communities versus 18,268 in reference communities. Intervention area children were predominantly white $(90 \%)$ and were less likely to have a governmental payer $(27.9 \%)$, whereas reference community children were more diverse (57\% white) and had more frequently a governmental payer (55.64\%). We observed lower overweight and obesity prevalence for intervention area children (29.7\% in 2011-2012 and 26.6\% in 2015-2016) compared to reference community children (33.4\% in 20112012 and $33.6 \%$ in 2015-2016). Reference community children more closely resembled national data.

Conclusion: Preliminary results suggest that children in the PowerUp and BearPower intervention communities have healthier overall BMI's than reference community children. Since PowerUp and BearPower were recently rolled out (2013-2015), data are not yet adequate to determine the impact of the intervention.

Keywords: child and adolescent health, demographics, health promotion, prevention, screening, evaluation research, racial/ethnic differences, health care organization

P7.03

Implementation of the Group Lifestyle Balance Program for Diabetes Prevention in Clinical Practice

Catherine Nasrallah, ${ }^{1}$ Nina Szwerinski, ${ }^{1}$ Vidita Chopra, ${ }^{1}$ Meghan Halley, ${ }^{1}$ Kristen Azar, ${ }^{1}$ Robert Romanelli ${ }^{1}$

${ }^{1}$ Palo Alto Medical Foundation, Palo Alto, CA

Background: The Group Lifestyle Balance ${ }^{\mathrm{TM}}$ (GLB) program is an adaption of the landmark Diabetes Prevention Program for community settings that has been approved by 
the Centers for Disease Control and Prevention as a model curriculum for type 2 diabetes prevention. The long-term impact of GLB is unknown when integrated into routine care within a health care system. The implementation of GLB across 4 largely autonomous and highly diverse Sutter Health regional affiliates in 2010 provides a unique opportunity to evaluate the program in a large multispecialty health care organization as a natural experiment. Herein, we report preliminary findings for the implementation of GLB across regional affiliates and clinic sites.

Methods: We used snowball sampling to identify GLB leaders and clinic facilitators within each region. We then conducted pilot interviews with these populations to inform future in-depth semi-structured interviews. Pilot interview questions focused on the GLB curriculum structure, participant costs, clinics resources, and workflows.

Results: We interviewed 4 GLB regional leaders and 8 clinic facilitators (each from a different site). Interviews revealed that the GLB program was implemented at 19 clinic sites within the 4 regions. At 8 sites included in this preliminary analysis, GLB is facilitated by a certified diabetes educator. The program is not restricted only to those with prediabetes, but most sites require individuals to have high cardiometabolic risk $(n=6)$. Participants are referred to the program by a physician or dietitian. Main differences in program implementation across clinics are related to the curriculum used (original University of Pittsburgh curriculum $[\mathrm{n}=5]$, newer Prevent T2 curriculum $[\mathrm{n}=2]$, or a hybrid curriculum $[n=1]$ ), the length (range: $3-12$ months) and total number of sessions per program (range: 9-25), the number of times the program was offered per year (range: $2-7$ ), and the annual enrollment costs to participants (range: \$0-\$688). An updated analysis, including the remaining 11 sites, will be presented.

Conclusion: This preliminary analysis suggests that although the GLB program was implemented in a single health system, there are important differences across and within regions. Such differences offer a natural experiment to understand how variation in the implementation of GLB impacts program effectiveness and participant outcomes.

Keywords: health promotion, prevention, screening, evaluation research, diabetes, health care organization, qualitative research

\section{P7.04}

\section{Assessment of Gaps in Major Components of Chronic Kidney Disease Care for Adults in a Primary Care Setting}

JoAnn Sperl-Hillen, ${ }^{1}$ Jay Desai, ${ }^{1}$ A. Lauren Crain, ${ }^{1}$ Patrick J. O'Connor, ${ }^{1}$ Kris Kopski, ${ }^{2}$ Rajeev Kaul, ${ }^{2}$ Christine Johns ${ }^{2}$

${ }^{1}$ HealthPartners Institute, Bloomington, $M N ;{ }^{2}$ Park Nicollet Medical Center, Minneapolis, MN
Background: There is substantial evidence that key components of chronic kidney disease (CKD) care slow progression of renal disease and substantially reduce the occurrence of major adverse cardiovascular (CV) events. We sought to determine if there are gaps in major CKD quality care components in a large Minnesota care system.

Methods: Electronic health record data was evaluated for adults age 18-79 years seen for office visits in one of 17 primary care clinics in 2015-2016. Patients who had at least one glomerular filtration rate (GFR) test that met criteria for stage 3-4 CKD (GFR: 15-59) were assessed for a) identification of a CKD diagnosis (through ICD-10 visit or problem list code), b) blood pressure (BP) control to $<140 / 90 \mathrm{mmHg}$ or $<130 / 80 \mathrm{mmHg}$, c) angiotensinconverting enzyme inhibitor (ACEI) or angiotensin receptor blocker (ARB) use if concomitant hypertension or increased albuminuria was identified, d) A1c control, and e) frequency of observed nephrology visits in the last 2 years for patients with more advanced stage $4 \mathrm{CKD}$ or stage $3 \mathrm{~b}$ CKD with increased albumin-to-creatinine ratio ( $>30 \mathrm{mg} / \mathrm{g})$.

Results: Of 5766 adults meeting GFR criteria for stage 3-4 CKD, 88.2\% had a hypertension diagnosis and only $49.2 \%$ had a CKD diagnosis; 67.2\% had a $\mathrm{BP}<140 / 90 \mathrm{mmHg}$ and $37.3 \%$ a $\mathrm{BP}<130 / 80 \mathrm{mmHg}$; and $57.8 \%$ were currently on ACEI/ARB. Of 35.8\% with diabetes, $76.7 \%$ had A1c $<8 \%$ and $50.3 \%$ had A $1 \mathrm{c}<7 \%$. Of 636 patients with a nephrology referral indication, $45.6 \%$ had seen a nephrologist. CKD was more likely to be diagnosed in patients with more advanced CKD ( $72 \%$ of stage $3 \mathrm{~b}$ CKD and $87 \%$ of stage 4 ), but rates of BP control, ACEI/ARB use, and glycemic control were not significantly higher for this population.

Conclusion: Quality improvement strategies are needed to overcome the sizeable gaps in quality of CKD care observed in this well-organized primary care setting. The use of clinical decision support tools that can identify patients with CKD and provide timely suggestions for treatment of key care components and referrals to nephrology has the potential to improve CKD patient outcomes.

Keywords: cardiovascular disease, primary care, ambulatory/outpatient care, clinical decision-making, chronic disease, clinical practice patterns/guidelines, quality improvement

\section{P7.05}

The Progression From Prediabetes to Diabetes Who Progresses and When?

Ning Fu, ${ }^{1}$ June O'Leary, ${ }^{1}$ Glenn Melnick, ${ }^{1}$ Joyce LaMori, ${ }^{2}$ Janelle Howe, ${ }^{3}$ Jeremy Rich 4

${ }^{1}$ University of Southern California, Los Angeles, CA; ${ }^{2}$ Janssen Scientific Affairs, LLC, Los Angeles, CA; ${ }^{3}$ DaVita HealthCare Partners, El Segundo, CA; ${ }^{4}$ HealthCare Partners Institute for Applied Research and Education, El Segundo, CA 
Background: In 2015, 34\% of U.S. adults (age 18+ years) were estimated to have prediabetes. Prediabetes is a state of intermediate hyperglycemia, not quite high enough to result in a diagnosis of type 2 diabetes. Diabetes is a progressive disease and the categorization "prediabetes" is used to identify a high-risk state for transitioning to diabetes. Better understanding of who progresses from prediabetes and when will enable development of targeted policies and interventions to potentially mitigate the public health impact of diabetes now and for generations.

Methods: Using multivariate regression we modeled the transition from prediabetes to diabetes over the 10 -year period beginning January 1, 2007, through December 31, 2016, using data from DaVita HealthCare Partners. American Diabetes Association hemoglobin A1c cut-points were used to group patients by glycemic level: diabetes $(\geq 6.5 \%)$, prediabetes $(5.7 \%-6.4 \%)$ or normal $(<5.7 \%)$. We studied the association of age, gender, and other known risk factors for diabetes, such as race and ethnicity, body mass index, hypertension, low high-density lipoprotein cholesterol and high triglycerides.

Results: Our preliminary analysis began with a baseline population of 48,119 in 2007 that had at least one hemoglobin A1c test result; $41 \%$ of this total had hemoglobin A1c values in the prediabetes range during 2007 (21\% were below $5.7 \%$, normal glycemic level, and 38\% were above $6.4 \%$, diabetes level). Of those patients with a prediabetes A1c level in $2007,39 \%$ (7505) were $\leq 65$ years and $61 \%(11,516)$ were $\geq 65$ years. The analysis then tracked this prediabetes population over time and found that 3 years later almost onethird of this population had transitioned to diabetes levels, and by the end of the period the transition rate reached $38 \%$. Initial results indicated higher transition rates occur among individuals living in lower-income Hispanic areas with limited English proficiency and that increasing age and female gender are significant predictors of the transition from prediabetes to diabetes within 10 years.

Conclusion: These findings are potentially important because they are based on a unique 10-year database of a very large population of commercially insured and Medicare-covered members who live in the ethnically and socioeconomically diverse greater Los Angeles area.

Keywords: observational study, chronic disease, epidemiology

\section{P7.06}

\section{Prevalence of Diabetes and Statin Treatment in Youth}

Elmor Pineda, ${ }^{1,2}$ Catherine McNeal, ${ }^{2}$ I-Chia Liao, ${ }^{2}$ Paul Godley $^{2}$

${ }^{1}$ University of Texas at Austin, Austin, TX; ${ }^{2}$ Baylor Scott \& White Health, Temple, TX

Background: Data from the National Health and Nutrition Examination Survey (NHANES) and SEARCH for Diabetes in Youth study have provided estimates of the populationbased prevalence of type 1 (T1D) and type 2 (T2D) diabetes among U.S. youth. This population of youth is at high risk for increased mortality and morbidity due to future atherosclerotic cardiovascular disease; as such, guidelines recommend lipid screening in children $\geq 10$ years of age soon after the diagnosis and recommend considering pharmacological treatment in those who continue to have low-density lipoprotein (LDL) cholesterol $>160 \mathrm{mg} / \mathrm{dL}$ or LDL cholesterol $>130 \mathrm{mg} / \mathrm{dL}$ despite medical nutrition therapy and lifestyle changes.

Methods: This is a retrospective, observational study assessing year-to-year trends using medical and pharmacy claims of enrolled youth age 2-20 years from 2001 through 2013. Proportions based on sample size and a positive diagnosis for diabetes were used to determine prevalence. Chi-squared tests for categorical data were used to compare changes in prevalence.

Results: A total of 172,407 members age 2-20 years were identified from 2001 to 2013. Overall prevalence of diabetes of any type increased significantly. In terms of age group, members $12-17$ years and 18-20 years showed a significant increase in prevalence of diabetes of any type. In terms of race/ ethnicity, prevalence of diabetes of any type for white and Hispanic members increased significantly. While there was not a significant increase in females, there was a significant increase in prevalence of diabetes of any type. Highest change in prevalence of statin treatment in youth with diabetes occurred from 2006 to 2007, with the highest occurring in 2008.

Conclusion: Our data show that from 2001 to 2013, overall prevalence of diabetes increased. As expected, prevalence for T1D was greater than T2D; however, both are steadily increasing. We observed minimal changes of the total number of youth with any type of diabetes year to year, likely as the result of kids aging out of the study, which could have underestimated our results. Prevalences for some races that are known to have high diabetes risk were unable to be determined due to our low enrollment of Asians/Pacific Islanders, Native Americans, and members of multiple races. Keywords: child and adolescent health, pharmacy, observational study, demographics, virtual data warehouse, diabetes, chronic disease, clinical practice patterns/ guidelines, epidemiology

\section{GENETICS, GENOMICS, PRECISION MEDICINE}

P8.01

Breast Cancer Screening and Prevention Risk Assessment-Based Programs: A National Environmental Scan

Dina Hassen, ${ }^{1}$ Susan Snyder, ${ }^{1}$ Alanna Rahm, ${ }^{2}$ Deanna Jarrett, ${ }^{3}$ Samantha Crissinger, ${ }^{3}$ Qiang Hao, ${ }^{1}$ Rosemary Leeming ${ }^{4}$ 
${ }^{1}$ Department of Epidemiology and Health Services Research, Geisinger Health, Danville, PA; ${ }^{2}$ Genomic Medicine Institute, Geisinger Health, Danville, $P A ;{ }^{3}$ Geisinger Clinic, Danville, PA; ${ }^{4}$ Geisinger Medical Center, Danville, PA

Background: Although there is some variation in leading guideline-issuing organizations' evidence-based breast cancer screening and prevention recommendations, a consensus has emerged supporting a paradigm shift from a one-size-fits-all to an individualized risk assessmentbased approach. This shift is reflected in the recent growth in programs commonly referred to as high-risk breast clinics (HRBCs). While intended to optimize patient outcomes, there is no formalized care model or research-based information describing or evaluating these programs, which are characterized by substantial variation. The objective of this research is to conduct a national environmental scan of HRBC programs to describe the current state and to use the results to formally define a new "Breast Risk Assessment using Cancer Evidence" (BRACE) care model to facilitate and evaluate implementation of risk assessment-based care.

Methods: A national environmental scan based on a survey of HRBC programs is being completed using systematic methods. Programs were identified primarily through web- and literature-based searches of publicly available information. A data collection instrument in the form of an emailed online and/or phone survey is used to obtain information on program characteristics based on attribute domains identified for a preliminary conceptual BRACE care model. Analysis of completed responses will encompass key program attributes within domains, such as target population, services offered, professional staff composition and roles, and program structure. Descriptive statistics will be calculated to report the variability in attributes and chi-squared tests will be used to test significance in attribute differences between HRBC program categories.

Results: More than $100 \mathrm{HRBC}$ programs have been identified for survey dissemination. The environmental scan results will be a synthesis of publicly available information and analysis of survey responses to characterize the current national landscape of risk assessment-based breast cancer screening and prevention programs, identify distinct program categories based on differences in key attributes, and develop an initial conceptual BRACE care model for dissemination.

Conclusion: The environmental scan findings are essential to understanding the implementation, variation, feasibility, barriers, and facilitators associated with recommended risk assessment-based breast cancer screening and prevention care, and to inform development of a scientific program evaluation framework to assess and improve implementation.

Keywords: cancer, genetics, genomics, health promotion, prevention, screening, quality improvement
P8.02

\section{Patient-Facing Precision Medicine: Educating Patients to Be Active in Their Care}

Rachel Wynn, ${ }^{1}$ Kristen Miller ${ }^{1}$

${ }^{1}$ National Center for Human Factors in Healthcare, MedStar Health, Washington, DC

Background: The prevalence of precision medicine (PM) - using genomic information to guide treatment of disease - is rising, and with it, the need for patient engagement. Patients engaged in their own care are equipped with the skills to help manage their own condition, are enabled to make decisions about their own care, and are empowered to ask relevant questions. For that to happen, patients must first understand PM, difficult even for providers. The complexity and quickly developing nature of PM creates unique challenges in educating patients to a level of understanding that allows for patient-centered care. To create effective tools for patient education, we must identify what information should be included, ensure that tools are tailored to health literacy level, reading level, and language of patients, and design them in a way that makes the information easy to access. We aimed to address the first of these questions, identifying the information patients require to be engaged in PM.

Methods: We created a survey that asked users to rate their understanding of PM concepts, their confidence in their ability to use that knowledge to make informed decisions about care, and the usefulness of tools currently used in MedStar Health cancer centers. To survey a wide population of people, we employed Amazon's Mechanical Turk, an online tool that allows for fast recruitment and payment to a global user base. We limited our survey to English-language users in the United States who have cancer or an immediate family member with cancer.

Results: Results of 200 respondents will be aggregated to identify themes of understanding or misunderstanding of the genetic concepts on which PM is based. We also identified themes regarding the perceived usefulness and accessibility of current tools. We will use results to identify strengths and weaknesses of current tools in their ability to address informational needs.

Conclusion: The complexities of PM and genetic mutations need to be clarified so that participation in PM is not hindered by misunderstanding. Future work will identify and evaluate existing tools and their ability to meet informational needs.

Keywords: cancer, quality of care, patient-provider communication 
P8.03

\section{Defining Phenotypes for Return of Genetic Results to Patients: Lessons From eMERGE III}

\author{
Jane Grafton, ${ }^{1}$ James Ralston, ${ }^{1}$ Aaron Scrol, ${ }^{1}$ David \\ Carrell ${ }^{1}$
}

${ }^{1}$ Kaiser Permanente Washington Health Research Institute, Seattle, WA

Background: A primary aim of the Electronic Medical Records and Genomics Network (eMERGE) is to integrate genomic information into clinical care through the electronic medical record (EMR) - including sequencing results for the 59 medically actionable genes per the American College of Medical Genetics and Genomics, which include colorectal cancer (CRC) genes - enabling providers to take clinical action, including genomic counseling. When recruiting biobank participants to participate in eMERGE, we identified patient samples identified as having a history of CRC. We initially required strong evidence of CRC for inclusion (SEER tumor registry) but progressively relaxed evidence requirements to achieve planned sample size for sequencing. While returning results to 289 consented patients identified as likely having CRC history, we uncovered two possibly misclassified as having CRC. We chart-reviewed these cases in the EMR (Epic). We investigated possible misclassification among other eMERGE enrollees consented for return of results whom were identified as likely having CRC.

Methods: Of 2400 sequenced eMERGE III participants, 533 were identified with CRC history. Criteria were CRC through tumor registry, or $1+$ diagnoses of $\mathrm{CRC}$ with associated procedure. Of 533 participants, 289 consented to return of results. To identify possible misclassification, the 289 participants were placed into four mutually exclusive groups. Three chart review groups had reasonable possibility of CRC misclassification: 1 ) $n=34$ patients with only 1 CRC diagnosis and no CRC mention on problem list; 2) $\mathrm{n}=43$ patients with 2+ CRC diagnoses and no CRC mention on problem list; and 3) $n=1$ patient with CRC on problem list and no CRC diagnosis. We did not review charts of patients in group $4(\mathrm{n}=211$ patients with $\mathrm{CRC}$ on problem list and $2+\mathrm{CRC}$ diagnoses), as misclassification seemed unlikely.

Results: EMR chart review outcome (CRC status) groups were based on evidence in chart for determining presence/absence of CRC: 1) CRC confirmed (34 of 78); 2) insufficient data in EHR (10 of 78); 3) colorectal neuroendocrine tumor on pathology report (6 of 78); 4) alternative diagnosis per pathology report (26 of 78); and 5) alternative diagnosis by chart review (2 of 78).

Conclusion: Level of certainty for returning clinical results is higher than needed for inclusion/exclusion from the phenotype algorithm. We explore options to revise the process of return of results to patients, including removal of indication of CRC on the lab report returned to providers, and omitting $\mathrm{CRC}$ indication from documents likely to become part of the patient chart.

Keywords: genetics, genomics, patient-provider communication
P8.04

Development of a Physician Survey on Genetics in Health Care, Research, and Precision Medicine Within Kaiser Permanente

\author{
Cabell Jonas, ${ }^{1}$ Michelle Turner, ${ }^{1}$ Kristen Janes,${ }^{2}$ Andrea \\ Burnett-Hartman, ${ }^{3}$ Erica Blum-Barnett, ${ }^{3}$ Heather \\ Clancy, ${ }^{4}$ Nazneen Aziz ${ }^{2}$
}

${ }^{1}$ Kaiser Permanente Mid-Atlantic States, Rockville, MD; ${ }^{2}$ Kaiser Permanente Program Office, Oakland, CA; ${ }^{3} \mathrm{Kaiser}$ Permanente Colorado, Denver, CO; ${ }^{4}$ Kaiser Permanente Northern California, Oakland, CA

Background: Remarkable advances in DNA sequencing technologies are enabling rapid analysis of a patient's DNA in clinical laboratories. However, despite the technological breakthroughs in clinical sequencing, the adoption of genomic knowledge in patient management for disease prevention, diagnosis, and treatment decision-making is lagging. Realizing advances requires a health care system that responds and works in collaboration with research to translate new knowledge into better outcomes. Nationwide, research programs such as Electronic Medical Records and Genomics Network (eMERGE), Clinical Sequencing Evidence-Generating Research (CSER), All of Us, and Geisinger Health's MyCode are sequencing healthy individuals to return certain genetic findings to research participants. Direct-to-consumer genetic testing companies also are delivering predisposition risks to consumers. Increasingly, physicians encounter patients' genetic testing results from outside the health care delivery system. To better understand the current clinical environment, physician knowledge and attitudes toward genetics, and the return of incidental findings both from genetic tests and research participation, we worked with Kaiser Permanente (KP) leadership, physicians, and experts to develop an online survey for KP physicians.

Methods: The survey team outlined five domains of interest and conducted a literature review to identify prior physician surveys. In addition to external experts, we engaged KP clinical experts in the Interregional Genetics Working Group, the Interregional Genetic Counselors Working Group, KP Research Bank, Permanente Medical Group, and Laboratory and Clinical Pharmacy. KP Washington survey consultants conducted two rounds of cognitive testing with physicians, which focused on clarity and relevance of content. Physicians also submitted feedback via email. We conducted two rounds of user acceptability testing to check online functionality on computer, phone, and tablet.

Results: Specific KP physicians (internal medicine, oncology, cardiology, obstetrics/gynecology, pediatrics) located nationwide will receive an invitation to complete the online survey. Results will inform strategies to develop new care pathways and infrastructure to ensure KP physicians are well prepared for use of genomic information in precision medicine. 
Conclusion: Including physicians and health system stakeholders throughout the survey development and testing process ensures the research insights will be relevant for precision medicine planning.

Keywords: genetics, genomics, survey research and methods

\section{P8.05}

\section{Kaiser Permanente Survey on Genetics in Research and Health Care}

Erica Blum-Barnett, ${ }^{1}$ Andrea Burnett-Hartman, ${ }^{1}$ Sarah Madrid, ${ }^{1}$ Cabell Jonas, ${ }^{2}$ Michelle Turner, ${ }^{2}$ Kristen Janes, ${ }^{3}$ Heather Clancy, ${ }^{3}$ Julie Harris-Wai, ${ }^{4}$ Nazneen $\mathrm{Aziz}^{3}$

${ }^{I}$ Institute for Health Research, Kaiser Permanente Colorado, Denver, CO; ${ }^{2}$ Regional Office, Mid-Atlantic Permanente Medical Group, Rockville, MD; ${ }^{3}$ Kaiser Permanente Program Office, Oakland, CA; ${ }^{4}$ University of California, San Francisco, San Francisco, CA

Background: The Kaiser Permanente Research Bank (KPRB) currently has approximately 287,000 members enrolled, with a goal to enroll 500,000 members total. Members who enroll in KPRB have consented to having their biospecimens and linked electronic health record used for genomic research as well as other bioassays. To better understand Kaiser Permanente (KP) member perspectives on return of genetic research results, KP investigators worked with a patient advisory panel (PAP) and other stakeholders to develop an online survey for KP members.

Methods: We outlined 12 domains of interest and conducted a literature review of published surveys and, when available, standard instruments for these domains. Simultaneously, we assembled a PAP of 6 advisors from the Colorado Patient Partners in Research (CoPPiR) network. Four in-person PAP meetings were held covering 1) project overview, 2) domain prioritization, 3) instrument selection, and 4) feedback on first draft of the survey. After incorporating PAP feedback, a second draft was circulated to scientific leaders on the KPRB leadership team, members of KPRB's community advisory and bioethics advisory boards, the KP Interregional Genetics Workgroup, and operational and clinical KP leaders. Then, a third draft was circulated to the PAP, reviewers from KP Office of Diversity, Equity, and Inclusion, the KP Washington Survey Research Program, and KP Mid-Atlantic States literacy specialists to simplify the survey reading level. Finally, the survey was converted into an online version, and the team conducted two rounds of user acceptability testing for clarity and usability of the online survey.

Results: From 7 KP regions, 10,000 KPRB participants and $100,000 \mathrm{KP}$ members who are not participating in the KPRB will receive the survey invitation via email. A \$5 Amazon gift code will be provided to participants who complete the survey. In combination with the results of a parallel physician survey, the results of the member survey will help to develop KPRB's policies on the return of genetic results and inform an overall precision medicine strategy for KP.

Conclusion: Including patient advisors and other health system stakeholders throughout the survey development process ensured that the interests of those who may be most impacted by the survey results are represented.

Keywords: genetics, genomics, survey research and methods, engagement of stakeholders

\section{HEALTH DISPARITIES}

\section{P9.01}

The Progression From Prediabetes to Diabetes Impact of Social Determinants of Health

June O'Leary, ${ }^{1}$ Ning Fu, ${ }^{1}$ Glenn Melnick, ${ }^{1}$ Joyce LaMori, ${ }^{2}$ Janelle Howe, ${ }^{3}$ Jeremy Rich $^{4}$

${ }^{1}$ University of Southern California, Los Angeles, CA; ${ }^{2}$ Janssen Scientific Affairs, LLC, Los Angeles, CA; ${ }^{3}$ DaVita HealthCare Partners, El Segundo, CA; ${ }^{4}$ HealthCare Partners Institute for Applied Research and Education, El Segundo, $C A$

Background: It has been said "your zip code matters more than your genetic code" when discussing the impact of a person's neighborhood on life expectancy in the U.S. This finding is embedded in the social determinants of health - factors like education, income, and race, which impact where people live and their health outcomes. There is limited evidence of the importance of social determinants of health to the risk for developing type 2 diabetes. Methodological limitations and varying endpoints make it difficult to generalize or reach clear conclusions regarding the relative importance of different factors. We utilize a large and diverse sample of patients from an integrated care system in the greater Los Angeles area (DaVita HealthCare Partners) to investigate the impact of several social determinants of health on the likelihood of transitioning from prediabetes to diabetes. Prediabetes, in which blood glucose levels are elevated above normal levels but not quite high enough to be considered diabetes, is a high-risk state for developing diabetes. It was recently estimated that one-third of younger adult Californians, those age 18-39 years, have prediabetes. Methods: Using Census zip code-level data from the American Community Survey, we identified 10 social determinants of health that have been found to be associated with type 2 diabetes. These zip code-level variables were included in a multivariate regression model of the transition from prediabetes to diabetes, controlling for several individual-level risk factors.

Results: For 2007, 19,021 patients were identified with a prediabetes hemoglobin A1c level (5.7\% to 6.4\%); for 2018, 
an additional 12,673 were identified. Descriptive analysis of zip codes suggested that higher rates of transition over the 10-year study period occurred in less affluent, Hispanic neighborhoods. After controlling for multiple individuallevel risk factors, our preliminary analysis indicated small but statistically significant associations between transitioning from prediabetes to diabetes with neighborhood educational attainment, household income level, and home value.

Conclusion: Further investigation into the social determinants of health and their role in the development and progression of diabetes may provide opportunities for the health care system to cost-effectively target resources.

Keywords: geographic/spatial factors, social determinants of health, diabetes, small-area analysis

\section{P9.02}

\section{Understanding Diabetes Management Among Hispanics at Kaiser Permanente Northwest}

Dea Papajorgji-Taylor, ${ }^{1}$ Jennifer Schneider, ${ }^{1}$ Inga Gruss, ${ }^{1}$ David Mosen, ${ }^{1}$ Nangel Lindberg ${ }^{1}$

${ }^{1}$ Kaiser Permanente Center for Health Research, Portland, OR

Background: The Kaiser Permanente Northwest (KPNW) region has reported a $12.6 \%$ gap in rates of glycemic control between Hispanic and non-Hispanic white patients, with a lower proportion of Hispanic patients showing adequate levels of glycemic control (A1c > 8\%). While recognizing that the term "Hispanic" is used to describe a highly heterogeneous population of diverse sociocultural backgrounds, it is critical to identify nuanced and contextualized factors that contribute to this gap in health outcomes, particularly in the context of a comprehensive medical care organization. Relying on qualitative data collection, this study explores sociocultural factors that might help explain suboptimal levels of diabetes control in this population. The goal is to identify actionable elements to improve care and support for patient self-care.

Methods: The study team has partnered with a KPNW primary care provider (PCP) panel serving Hispanic patients diagnosed with type 2 diabetes to conduct a qualitative pilot study. From the PCP's Hispanic patient panel, individuals with A1c $>8 \%$ will be invited to participate, which involves direct observation of medical encounters between PCPs and patients as well as individual in-depth telephone interviews with both patients and PCPs. Observations and interviews will center on identification of factors that may help optimize patient-provider communication and patient adherence to diabetes self-care recommendations.

Results: This is an ongoing study. By the expected completion date (December 31, 2017), we hope to provide thematic summaries regarding Hispanic patients' experience in receiving diabetes care at KPNW, barriers and facilitators of optimal patient-provider communication, patient diabetes self-care, and adherence to treatment recommendations.
Conclusion: Conclusions from this study will identify gaps in equitable care and inform efforts to improve culturally competent practice in diabetes care.

Keywords: cardiovascular disease, observational study, primary care, patient-provider communication, engagement of stakeholders, racial/ethnic differences, social determinants of health, diabetes, qualitative research, chronic disease, patient experience/satisfaction

\section{P9.03}

Use of Interpreter Flag in Determining Eligibility for Studies Conducted at Kaiser Permanente Washington Health Research Institute: Mining Information From the Survey Research Program

\section{Paula Sandler, ${ }^{1}$ Luesa Jordan, ${ }^{1}$ Matthew Nguyen, ${ }^{1}$ Megan Baldwin ${ }^{1}$}

${ }^{1}$ Kaiser Permanente Washington Health Research Institute, Seattle, WA

Background: As a shared resource for Kaiser Permanente Washington Health Research Institute, the Survey Research Program (SRP) contacts thousands of participants annually via phone, mail, and web-based surveys. Inevitably, there are participants unable to participate either because they are non-English speaking or hearing impaired. Since 2014, only one of more than 50 fielded phone room studies used Spanish-bilingual interviewers. Teletypewriter and/ or video relay services are available to participants with a hearing impairment, but the participant must initiate use of the technology. The electronic health record (EHR) has an "Interpreter flag" field that identifies members requiring an interpreter, including American Sign Language. Some studies utilize the Interpreter flag as an exclusion criterion. Together, this tells us that non-English speaking and hearing-impaired individuals may not be invited to participate in studies or, if invited, not able to participate. Using data in the SRP database, augmented by information from the virtual data warehouse (VDW), we will investigate how the Interpreter flag is currently used and how to better utilize it in the future. Methods: We identified approximately 50,000 unique participant records from 24 studies in which the SRP attempted telephone contact from 2014 to present. We used the VDW to obtain the Interpreter flag status for our sample. We used process and outcome variables (call disposition, study type, final status), basic demographic variables (date of birth, gender, ethnicity, race), and Interpreter flag (checked vs unchecked) to determine: Is the interpreter flag a predictor of final outcome? (complete vs "ineligible: language," "ineligible: hearing impaired," and "refused, unable to reach"). Seven of the 24 studies used the Interpreter flag as one of their exclusion criteria. How did response rates differ between those studies and studies that did not use that exclusion? What is the age, race/ethnicity, and gender distribution of those with an Interpreter flag? 
How often did SRP initiate contact with individuals who could not participate due to language barriers or hearing impairments? Could those individuals have been preidentified by the Interpreter flag? Could study materials or procedures be tailored to allow for individuals with these barriers to participate?

Results: Across all 24 studies, only $2.1 \%$ of the participants were determined to be ineligible for study participation compared to $21.8 \%$ for those with an Interpreter flag. Of the 1067 who were determined to be ineligible, $328(30.7 \%)$ were due to hearing and language barriers. Nearly all of those with an Interpreter flag were screened out due to language. Completed interviews or successful recruitment were achieved for $40.6 \%$ of those having an Interpreter flag as compared to $44.8 \%$ of participants without. A smaller percentage of respondents with the flag refused or were unable to be reached, suggesting that, if enabled, they might be more likely to participate than those without a flag.

Conclusion: Participants with an Interpreter flag were much more likely to be determined ineligible for study participation, and almost all of those identified as ineligible were so due to a language barrier. While $25.3 \%$ of all participants were ineligible due to language barriers, this was $98 \%$ of those with the Interpreter flag.

Keywords: survey research and methods, demographics, access to services

\section{P9.04}

Depression Screening and Education: Options to Reduce Barriers to Treatment (DESEO): Primary Outcomes From a Culturally Adapted Education Intervention to Increase Treatment Engagement Among Hispanics in Primary Care

\section{Katherine Sanchez ${ }^{1}$ \\ ${ }^{1}$ Baylor Scott \& White Health, Dallas, TX}

Background: In 2014, the Centers for Medicare \& Medicaid Services' Hispanic Health Services Research Grant Program funded the Depression Screening and Education: Options to Reduce Barriers to Treatment (DESEO) project to implement an education intervention designed to increase disease literacy and dispel myths about depression and its treatment among Hispanic patients, thus reducing stigma and increasing treatment engagement.

Methods: Universal depression screening was implemented for all adult patients during visits with their primary care providers using the 9-item Patient Health Questionnaire (PHQ-9). Depressed patients (PHQ-9 score > 10) then completed the 7-item Generalized Anxiety Disorder assessment (GAD-7) and were invited to participate in the study. DESEO utilized a one-group pretest-posttest design to assess a culturally adapted depression education intervention (a comic book-style fotonovela) within a sample of 350 depressed Hispanic patients. The Depression
Knowledge Measure (DKM) was used to evaluate the effect of the education. Three stigma measures were used to assess stigma associated with depression. Statistical tests of the demographics were completed and differences examined. Various bivariate statistical tests, including t-tests, chisquared $\left(\chi^{2}\right)$, and Fisher's exact, were used for changes in participant-reported scores over time as well as repeatedmeasures analysis of variance. Multinomial logistic regression was used to test the association between the selfreport measures from follow-up and the likelihood of the treatment-engagement categories.

Results: Overall, participants reported significantly lower scores for self-reported depression, anxiety, and mental health stigma measures at second session and at 30-day follow-up compared to baseline. These changes included average PHQ-9 scores decreasing from 17.53 at baseline to 9.47 at follow-up $(\mathrm{P}<0.001)$, GAD-7 scores decreasing from 12.97 to $7.62(\mathrm{P}<0.001)$, and Stigma Concerns About Mental Health Care decreasing from 0.43 to $0.10(\mathrm{P}<0.001)$. Additionally, participants reported increased knowledge in depression but also greater concern and negative perceptions about antidepressant medication. Finally, a majority of participants reported entering some form of treatment at follow-up (273 of 305, 89.5\%).

Conclusion: DESEO addressed the need for early identification of depression, and the culturally adapted education intervention increased knowledge of the disorder, reduced stigma, and led to significant engagement in treatment, which has implications for reducing the morbidity of related medical illnesses.

Keywords: primary care, behavioral and mental health, racial/ethnic differences, social determinants of health, social work

\section{HEALTH POLICY}

P10.01

A Prospective, Randomized Study of Fixed Versus Variable Dosing of 4-Factor Prothrombin Complex Concentrate for Emergent Warfarin Reversal at a Large Tertiary Care Medical Center

\section{Zachary Stoecker, ${ }^{1}$ Brandon Van Amber, ${ }^{2}$ Kurt Isenberger, ${ }^{1}$ Robert LeFevere, ${ }^{1}$ Casey Woster, ${ }^{1}$ Alexia Terwilliger, ${ }^{1}$ David Dries ${ }^{1}$ \\ ${ }^{1}$ Regions Hospital, Saint Paul, MN; ${ }^{2}$ University of Minnesota, Minneapolis, $M N$}

Background: 4-factor prothrombin complex concentrate (4FPCC), a U.S. Food and Drug Administration (FDA)approved medication for warfarin reversal, contains all the vitamin K-dependent clotting factors inhibited by warfarin, making it desirable for emergencies. Optimal dosing of 4FPCC is currently unknown despite multiple studies. The 
FDA-approved dosing is 25 to $50 \mathrm{IU}$ factor IX per kilogram of body weight, dependent on international normalized ratio (INR). Regions Hospital recorded 67 4FPCC administrations from January 2015 to May 2016. Mean dose administered was 3021 IU (range: 1000-7000 IU), with an average cost of $\$ 7,236.17$ (range: $\$ 2,530-\$ 17,710$ ) per dose. If clinically effective, a lower, fixed dose may cost less and allow for more rapid administration compared to the FDA-approved variable dosing.

Methods: A prospective randomized trial is being conducted to determine if fixed-dose 4FPCC is noninferior to variable dosing for emergent warfarin reversal. Eligible patients are randomized $1: 1$ to receive either a fixed or variable dose of 4FPCC. This study utilizes a permuted block randomization schedule to allow for equal allocation of subjects. All enrolled subjects were evaluated for thromboembolic events at day 7 .

Results: Fisher's exact test was performed to assess the primary endpoint (INR reduced to $\leq 1.5$ after initial dose) using a noninferiority approach and a 95\% confidence interval. A total of 23 patients have been randomized 12 to fixed and 11 to the weight-based dose. A total of 5 patients did not achieve an INR $\leq 1.5 ; 4$ of these patients were in the fixed-dose group, while 1 was in the variabledose group. No statistically significant difference was seen between the groups $(\mathrm{P}=0.1854)$. A Wilcoxon rank-sum test was performed to compare cost between the two treatment groups. Median medication cost of the fixed-dose was lower (\$2,529.27; interquartile range [IQR]: 63.59) than the median cost of the weight-based dose $(\$ 3,372.36$; IQR: 1614) $(\mathrm{P}<0.0001)$.

Conclusion: It is important to note that of the 5 subjects who did not meet the primary endpoint, only 1 did not achieve an INR of $\leq 1.7$. No subjects were considered treatment failures, requiring additional 4FPCC. A fixed-dosing strategy could benefit patients in need of emergent anticoagulant reversal if proven to be noninferior to the variable-dosing strategy.

Keywords: clinical decision-making, research findings, clinical practice patterns/guidelines

\section{HEALTH SERVICES UTILIZATION, DELIVERY, AND COVERAGE}

P11.01

\section{Community Paramedic Partnership: Shifting Health Care Utilization Through Partnership Between Municipal Fire/Emergency Medical Services and the Local Level I Trauma Center}

Tia Radant, ${ }^{1}$ Joseph Pasquarella, ${ }^{2}$ Ann Majerus, ${ }^{3}$ Matthew Simpson, ${ }^{3}$ Adam Mayer, ${ }^{2}$ Sandi Wewerka, ${ }^{2}$ Paula Miller, ${ }^{2}$ Aaron Burnett ${ }^{2}$

${ }^{1}$ Regions Hospital Emergency Medical Services, Saint Paul, MN; ${ }^{2}$ Regions Hospital, Saint Paul, MN; ${ }^{3}$ Saint Paul Fire Department, Saint Paul, MN
Background: A partnership between a Level I trauma center and an urban municipal fire/emergency medical services (EMS) department for patients with congestive heart failure (CHF) was launched in 2014. The program aimed to improve health care utilization and reduce readmissions through a unique community paramedic (CP) partnership. We hypothesized that patients who receive $\mathrm{CP}$ visits for 30 days postdischarge have reduced rate of readmission and increased use of clinic visits.

Methods: Inpatients with admission diagnoses of $\mathrm{CHF}$, chronic obstructive pulmonary disease, or acute myocardial infarction were offered visits by a CP for up to 30 days postdischarge. Patients with homecare services upon discharge were excluded. Patients were required to sign a written informed consent form prior to participation. The $\mathrm{CP}$ visited the patient in the home 1-2 times per week for 4 weeks following discharge. At each visit, the $\mathrm{CP}$ conducted medication reconciliation, a physical examination, home safety evaluation, coordination of followup care, and referral to community or health care resources as needed. Pre/post comparisons were analyzed descriptively using means and standard deviations. Scores were assessed with Wilcoxon signed-rank tests.

Results: Between February 2015 and July 2017, 64 patients were enrolled; 32 patients completed the program with complete data. A comparison of 90-day health care utilization pre- and postadmission showed that patients who were provided CP services had a significant decrease in hospital admissions $(68 \%, \mathrm{P}<0.0001)$ and emergency department (ED) visits $(62 \%$, $\mathrm{P}<0.0001$ ), and had a $14 \%$ increase in clinic visits (ns, $\mathrm{P}=0.45$ ). A group of patients who met inclusion criteria but declined consent to participate was compared to the patient group that participated in the CP program. Patients who completed the program had a significantly higher decrease in admissions $(\mathrm{P}=0.0145)$ and $\mathrm{ED}$ visits $(\mathrm{P}=0.0009)$ pre- to posthospitalization than those who did not enroll $(n=20)$. There was no significant difference in change in clinic utilization.

Conclusion: Partnership between fire-based EMS and hospitals for community paramedic programs can be successful. CPs providing postdischarge care results in a shift of health care utilization toward reduced admissions/ED visits and increased clinic visits. Further research with a larger cohort is needed to determine if utilization patterns would be sustained past 90 days. Keywords: program evaluation, primary care, quality of care, patient-provider communication, complex disease management, multiple chronic conditions, health care costs, resource use, hospital, access to services, incentives

\section{P11.02}

Impact of Dental Provider Recommendations on Adolescent Vaccination Rates

Allison Naleway, ${ }^{1}$ Michelle Henninger, ${ }^{1}$ Lisa Waiwaiole, ${ }^{1}$ Michael Leo, ${ }^{1}$ David Mosen, ${ }^{1}$ Daniel Pihlstrom ${ }^{2}$

${ }^{1}$ Kaiser Permanente Northwest, Portland, OR; ${ }^{2}$ Permanente Dental Associates, Portland, OR 
Background: Dental visits provide an opportunity for recommending adolescent vaccines and improving coverage, but dental providers have not traditionally played a role in recommending vaccinations.

Methods: We conducted a cluster-randomized trial to evaluate whether dental provider recommendations would improve adolescent vaccination rates. We randomized 16 Kaiser Permanente Northwest dental clinics to intervention or usual care. Intervention clinic staff received training about adolescent vaccinations and were asked to hand out a brochure and verbally recommend vaccination. Dental staff in the usual care clinics provided only a written reminder of vaccinations that were due. To assess the impact of the intervention, we compared pre/postintervention changes in adolescent vaccination rates between intervention and control clinics and accounted for the clustering of patients within clinics using a mixed-effects logistic regression model. The preintervention period was October 2014September 2015, and the postintervention period was October 2015-September 2016.

Results: In the preintervention period, 11,613 adolescents 11-17 years of age had a dental visit and were included the analysis; the postintervention period included 12,031 adolescents. During the intervention period, adolescent vaccination rates increased at both intervention and control clinics. No significant differences in the rate changes were observed between the intervention and control clinics for tetanus-diphtheria-acellular pertussis (Tdap) vaccination (odds ratio [OR]: 1.10, 95\% CI: 0.91-1.33), meningococcal conjugate vaccination (OR: 1.12, 95\% CI: 0.97-1.30), human papillomavirus (HPV) initiation (OR: 1.03, 95\% CI: 0.92-1.17), second-dose HPV vaccination (OR: 1.00, 95\% CI: 0.89-1.11), or third-dose HPV vaccination (OR: 0.99, 95\% CI: 0.89-1.09).

Conclusion: Although vaccination rates improved overall among adolescents, the observed changes in vaccination rates did not differ in intervention and control clinics after dental providers were encouraged to make vaccine recommendations to their adolescent patients.

Keywords: child and adolescent health, health promotion, prevention, screening, dental care

\section{P11.03}

\section{Engaging With the "Other:" Lessons From Research to Inform Operations and From Operations to Inform Research}

\section{Su-Ying Liang, ${ }^{1}$ Harold S. Luft ${ }^{1}$ \\ ${ }^{1}$ Palo Alto Medical Foundation Research Institute, Palo Alto, $C A$}

Background: Researchers embedded within health care delivery systems can conduct studies using a wealth of routinely collected data from the system, share findings with the operations team for quality improvement, and receive feedback informing their research. This study illustrates lessons learned from the embedded researcher and the operation team partnership.

Methods: Using longitudinal administrative and electronic health record (EHR) data of a large ambulatory group practice, the research team conducted a series of analyses examining the association between physician practice patterns and resource use, outcomes, and patient experience. The study population included 242 primary care physicians (PCPs) (726 PCP-years) from 2011 to 2013. Phase 1 identified PCPs who dealt with multiple problems during acute visits ("max-packers"). Findings suggested that maxpacking is associated with better clinical quality, better patient-reported experience, and lower annual per-patient costs (all risk-adjusted), but more physician time using the EHR system. Phase 2 explored factors associated with PCPs who were max-packers, including practice processes (eg, scheduled appointment blocks), physician characteristics, patient-panel characteristics, and fixed effects (to account for the organization's 4 historically distinct regions), with standard errors adjusted for correlation over time for the same PCP. Presentation of early findings to operational leads led to additional variables to consider.

Results: Female PCPs and appointment blocks allowing more time for a visit were strong predictors of being a maxpacker. Other physician characteristics (years of practice, specialty), physician clinical FTE, and patient-panel comorbidity were not significant. PCPs in Region 4 were substantially more likely to be max-packers (odds ratio: 4.68, 95\% CI: 2.15-10.18). The operation team suggested that access issues in Region 4 could lead PCPs to avoid return visits. The model was revised to incorporate an access metric, attenuating the Region 4 effect (odds ratio: 2.99, 95\% CI: 1.33-6.74). The operational team now plans to conduct interviews with max-packers to learn their "secret sauce," after the research team revised the institutional review board protocol to allow the identification of max-packers.

Conclusion: Collaborations between research and operations may uncover organizational "insights" to improve research, and vice versa

Keywords: quality of care, health care organization, costs, resource use

P11.04

Implementing Smoking Cessation Counseling for Lung Cancer Screening-Eligible Patients

\author{
Jiang Li, ${ }^{1}$ Sukyung Chung, ${ }^{1}$ Harold S. Luft ${ }^{1}$ \\ ${ }^{1}$ Palo Alto Medical Foundation Research Institute, Palo \\ Alto, $C A$
}

Background: Smoking cessation counseling has long been recommended for heavy smokers, but uptake has been very low. The new recommendation and coverage of lung cancer screening with low-dose computed tomography (LCS- 
LDCT), which requires smoking cessation in parallel, has the potential to increase the use of smoking cessation counseling. Our aim was to assess the utilization of smoking cessation counseling among LCS-LDCT-eligible patients following the policy changes.

Methods: We analyzed the electronic health records data from a large health care system in Northern California during 2010-2016. Patients age 55-80 years who made at least one visit to a primary care provider each year and had no history of lung cancer at the time of visit (557,409 patientyears over 7 years) were included in the analysis. Trends in documentation of smoking history, number of eligible patients, and orders of smoking cessation counseling were assessed. Patient-level and provider-level factors associated with smoking cessation counseling orders during 2014-2016 were explored using hierarchical generalized linear models. Results: Documentation of smoking history has increased over time, from $61.5 \%$ in 2010 to $79.2 \%$ in 2016 . Smoking cessation counseling orders among current smokers who were eligible for LCS-LDCT have increased from 5.3\% in 2010 to $7.7 \%$ in 2016. Implementation of smoking cessation counseling varied considerably across primary care providers. Current smokers $75-80$ years old (odds ratio [OR]: $0.5,95 \%$ CI: $0.4-0.8)$ were significantly less likely to receive smoking cessation counseling orders compared to those 55-64 years old. Heavier smokers with 50-59 pack-years history of smoking were more likely to receive an order than lighter smokers with 30-39 pack-years (OR: 1.7, 95\% CI: 1.2-2.4). Current smokers seeing their regular primary care provider also were significantly more likely to receive a smoking cessation counseling order (OR: 2.0, 95\% CI: 1.3-3.1).

Conclusion: Future interventions to improve smoking cessation counseling among LCS-LDCT-eligible patients may be more effective if they focus on accurate documentation of smoking history and target lighter smokers who are not visiting their usual PCP. Barriers and facilitators of implementing smoking cessation counseling at patient, provider, and systems levels need to be better understood to inform targeted interventions.

Keywords: cancer, primary care, health promotion, prevention, screening, hierarchical modeling, clinical practice patterns/guidelines

\section{P11.05} Creating a Valid, Reliable, and Actionable Measure of
Current Population Health for Use by Health Plans

\author{
Thomas Kottke, ${ }^{1}$ Jason Gallagher, ${ }^{1}$ Marcia Lowry, ${ }^{1}$ \\ Sachin Rauri, ${ }^{1}$ Juliana Tillema, ${ }^{1}$ Jeanette Y. Ziegenfuss, ${ }^{1}$ \\ Nico Pronk, ${ }^{1}$ Susan Knudson ${ }^{1}$ \\ ${ }^{1}$ HealthPartners, Minneapolis, $M N$
}

Background: In 2008, Berwick, Nolan, and Whittington proposed the Triple Aim as a measure of health care system performance. While reliable, valid, and actionable measures of experience and cost have been developed for health plans, a measure of population health with the same three attributes has not. Therefore, we developed a method by which disability-adjusted life years (DALYs) attributable to a population and classes of conditions (musculoskeletal, psychosocial, neurological, etc) can be calculated using insurance claims and death records.

Methods: The Johns Hopkins $\mathrm{ACG}^{\circledR}$ System groups insurance claims into expanded diagnostic clusters (EDCs) and further groups EDCs into major EDCs (MEDCs). The conditions listed above are examples of MEDCs. We matched a Global Burden of Disease (GBD) project condition score to weight the health impact of each EDC and rolled the EDC-level DALYs up to the MEDC level to calculate the morbidity DALYs attributable to each MEDC for our 2011, 2012, and 2013 commercial members. We ranked MEDCs by DALYs, levels of function and levels of health reported by the members on a health assessment, and cost to the health plan attributable to each MEDC. We calculated correlation coefficients as below. We also calculated correlation coefficients for the number of MEDCs for which the member had claims with their function and health.

Results: Measures for years 2011/2012/2013, respectively: $72,693 / 58,434 / 61,723$ number of members; -0.24/-0.24/0.23 coefficient for GBD-weighted MEDC self-reported function and $-0.20 /-0.19 /-0.19$ for self-reported health; $-0.14 /-0.19 /-0.19$ coefficient for cost attributable to MEDC self-reported function and $-0.13 /-0.15 /-0.15$ for self-reported health; $-0.21 /-0.21 /-0.21$ coefficient for MEDCs per member self-reported function and -0.20/-0.20/-0.20 for self-reported health. *All coefficients are statistically significant.

Conclusion: The correlations between DALYs and selfreported function and self-reported health indicate that the measure is valid. Assessing population health with DALYs also is superior to assessing population health by analyzing the costs attributable to an MEDC. The fact that the performance of the DALYs is similar to the performance of the measure based on MEDCs per member also supports its validity. The year-over-year consistency of the correlation coefficients indicates that the measure is reliable.

Keywords: quality of care, managed care (features), health care organization, quality improvement

\section{P11.06}

Transgender Response and Quality of Care Study at Kaiser Permanente Mid-Atlantic States: Data Quality Metrics

Suma Vupputuri, ${ }^{1}$ Alphonse Derus,,${ }^{1}$ Christine Truong,,${ }^{1}$ Ayanna Wells, ${ }^{1}$ E.W. Emanuel ${ }^{1}$

${ }^{1}$ Kaiser Permanente Mid-Atlantic States, Rockville, MD

Background: It has been suggested that transgender (TG) patients have poorer health than cisgender patients; however, the quality of TG health care is unknown. The purpose of this 
study is to describe the transgender patient population at Kaiser Permanente Mid-Atlantic States (KPMAS) and develop quality health metrics to initiate the process of assessing quality of care. Methods: We included patients who were reported by the KPMAS HealthConnect ${ }^{\circledR}$ TG registry, which identifies active patients using TG-related ICD codes, TG procedures, and surgical history. We describe here the TG population at KPMAS with respect to demographics, how they were identified as TG, whether they had a TG procedure/surgery, and medications. Preliminary TG quality health metric domains include health care utilization, use of preferred providers, health system engagement, and medication fills. Additional metrics to be presented will include utilization of video and telephone visits, canceled appointments, number of hormone orders vs fills, and health maintenance and preventive screenings.

Results: We examined 282 current patients identified by the KPMAS TG registry on October 16, 2017. The mean age of this patient cohort was 33 years. There were $49 \%$ nonHispanic white, 22\% non-Hispanic black, 8\% Hispanic, and 2\% Asian/Pacific Islander. Approximately equal numbers of patients had procedures indicating a female-to-male versus male-to-female TG status. (Natal sex, recorded from administrative data, showed 57\% female). Among the cohort, $77 \%$ patients were identified by at least $2 \mathrm{TG}$ diagnoses, and $71 \%$ were identified by 1 diagnosis from either a behavioral health or endocrinology encounter. Health care utilization during a 2-year period included a median of 14 outpatient encounters (interquartile range [IQR]: 8-22); 16 email or telephone encounters (IQR: 7-28); and negligible inpatient and emergency room encounters. TG-preferred providers were seen by $70 \%$ of patients. In terms of patient engagement, the median number days that patients signed on to KP.org was 38 (IQR: 17-63). (Additional metrics are in process.)

Conclusion: It is important to establish baseline descriptors and quality indicators of the TG population at KPMAS to help clinical administrators identify and resolve potential care gaps. At KPMAS, the TG population seems to be a young, highly engaged patient population, with average health care utilization patterns.

Keywords: quality of care, gender/sex differences, health, health care, quality improvement

\section{P11.07}

Provider Utilization and Experience With an Embedded Approach to Integrated Behavioral Health Care for Adolescents

Kimberly Erlich, ${ }^{1}$ Daniel Becker, ${ }^{1}$ Ellis Dillon, ${ }^{2}$ Martina $\mathrm{Li}^{2}{ }^{2}$ Jinnan Li, ${ }^{2}$ Amy Heneghan ${ }^{3}$

${ }^{1}$ Mills-Peninsula Health Services, San Mateo, CA; ${ }^{2}$ Palo Alto Medical Foundation Research Institute, Mountain View, CA; ${ }^{3}$ Palo Alto Medical Foundation, Palo Alto, CA

Background: Mental health is a key determinant of overall well-being; $20 \%$ of adolescents have a behavioral disorder that impacts physical health, social relationships, and societal engagement. Unfortunately, up to $50 \%$ of those with behavioral disorders do not receive care. Reasons for this include poor access to behavioral health providers (BHPs), as well as primary care providers (PCPs) lacking training, comfort, and competence with providing behavioral health $(\mathrm{BH})$ care. Integrating $\mathrm{BH}$ services into primary care $(\mathrm{PC})$ may alleviate roadblocks to care, improve patient satisfaction and clinical outcomes, and increase PCP satisfaction. We developed and implemented an embedded $\mathrm{BH}$ service for adolescents as a model of PC-BH collaboration.

Methods: A BHP was embedded in two PC sites for 18 months, providing the following services collaboratively with PCPs: evaluation, treatment planning, medication management, and a cognitive behavioral therapy intervention (COPE). Electronic health records data were examined for service utilization. PCP surveys solicited opinions on service utilization and experience.

Results: A total of 178 eligible patients were referred to the embedded BHP in a 17 -month period by 28 out of 32 eligible PCPs (87.5\%), and 61.2\% (109) had at least 1 office visit. Of patients referred, $73.0 \%$ were female; mean age was 15 years. The most frequent reasons for referral were depression (44.4\%), anxiety (44.0\%), and stress (26.1\%). Of 16 PCPs surveyed, $87.5 \%$ agreed that the program enhanced clinical care and 93.8\% agreed that it enhanced patients' experience with care. PCPs rated the program favorably on referral process $(93.8 \%)$, communication with BHP $(100.0 \%)$, and overall experience with the service $(93.8 \%)$. Of PCPs surveyed, $81.3 \%$ stated they hoped the program would continue. Some PCPs requested clarification about guidelines for appropriate referrals.

Conclusion: Embedded BHPs is an efficient collaborative care model to improve access to $\mathrm{BH}$ services for adolescents. This model improves communication between BHPs and PCPs, enhancing both clinical care and patients' experience with care. Keywords: child and adolescent health, primary care, program evaluation, behavioral and mental health, access to services, ambulatory/outpatient care, quality improvement

\section{IMPLEMENTATION SCIENCE}

\section{P12.01 \\ Adapting an Outpatient Cancer Prevention Clinical Decision Support System to the Needs of a Large Rural Integrated Health Care System}

Melissa Harry, ${ }^{1}$ Daniel Saman, ${ }^{1}$ Patrick J. O'Connor, ${ }^{2}$ Heidi L. Ekstrom, ${ }^{2}$ JoAnn Sperl-Hillen, ${ }^{2}$ Joseph Bianco, ${ }^{3}$ Thomas Elliott ${ }^{2}$

${ }^{1}$ Essentia Institute of Rural Health, Essentia Health, Duluth, $M N ;{ }^{2}$ HealthPartners Institute for Education and Research, Bloomington, MN; ${ }^{3}$ Essentia Health-Ely Clinic, Ely, MN 
Background: Cancer is a leading cause of death in the United States. Reducing the incidence of common cancers through more consistent primary and secondary prevention in primary care is needed to achieve Healthy People 2020 goals. This paper focuses on preimplementation adaptations made to a National Institutes of Health-funded study that will integrate primary (tobacco cessation, obesity management, human papillomavirus vaccination) and secondary (breast, cervical, colorectal, lung) cancer prevention and shared decision-making tools (SDMTs) with an existing electronic medical record-linked and web-based cardiovascular clinical decision support system (CDSS) currently being tested in a large, rural integrated health care system based in the Midwest. To facilitate implementation and maximize utilization of the cancer prevention CDSS, the research team made several adjustments to the original study design in order to better adapt the intervention to fit the goals and culture of the integrated health care system. Our paper helps fill a gap in the literature, as few studies report on adaptations made to interventions based on feedback from intervention sites, particularly those that alter and enhance current CDSS. Methods: Guided by the Consolidated Framework for Intervention Research (CFIR), we analyzed data from preimplementation study documents and meeting minutes with health system providers and leaders and team meetings, along with leadership and provider key stakeholder interviews $(\mathrm{n}=28)$, focusing on preimplementation adaptations made to the study design and to the cancer prevention CDSS. We present those adaptations here, categorized by relevant CFIR domain.

Results: Based on feedback received from health care system providers and leaders, we made adaptations related to CFIR domains intervention characteristics, inner setting, outer setting, and process. In brief, we modified one intervention arm to focus on SDMTs rather than expanding the role of clinic medical assistants and adapted some content in the cancer prevention CDSS, our study design, and SDMTs to meet the needs of the delivery system in which the study is being conducted.

Conclusion: The strategies we used to better adapt the cancer prevention CDSS to the evolving needs of the integrated health care system may provide useful guidance and reference for other researchers implementing similar systems, including those that expand and enhance current CDSS.

Keywords: cancer, primary care, qualitative research, engagement of stakeholders, clinical decision-making, dissemination, implementation, innovation, clinical trials

\section{P12.02}

Hepatitis C Cascade of Care at Kaiser Permanente Mid-Atlantic States: The Laboratory Perspective

Cabell Jonas ${ }^{1}$

${ }^{1}$ Mid-Atlantic Permanente Research Institute, Rockville, MD

Background: Chronic hepatitis $\mathrm{C}$ virus (HCV) affects the large Baby Boomer-age population and those at risk due to blood exposure (such as those who use intravenous drugs). A chronic $\mathrm{HCV}$ infection is detected through a positive $\mathrm{HCV}$ antibody $(\mathrm{Ab})$ test, followed by a positive HCV RNA (viral load) laboratory test. Of patients who test positive for $\mathrm{HCV} \mathrm{Ab}$, the literature shows that up to half are never tested for HCV RNA, meaning a chronic HCV diagnosis cannot be established. The gap between HCV Ab and HCV RNA testing persists in part due to patient failure to return for a second blood draw after the initial positive $\mathrm{HCV}$ Ab result or incomplete ordering of HCV tests by providers.

Methods: In 2014, Kaiser Permanente Mid-Atlantic States implemented a new HCV screening pathway specifically designed to streamline laboratory testing for patients and ensure a complete set of diagnostic tests are easily ordered for the patient. Scalable resources, including laboratory workflow tools and training guides, have been outlined to promote adoption of this pathway by other Kaiser Permanente regions. The new HCV Pathway simplifies physician workflows and closes known care gaps through the use of technology, laboratory reflex testing, and a HCV care coordinator. Within the HCV Pathway, laboratory reflex testing ensures that all patients receive HCV Ab, HCV RNA, hepatitis B surface antigen, and human immunodeficiency virus (HIV)-1/2 testing from the blood samples collected from a single patient visit. If diagnosed as having chronic $\mathrm{HCV}$ (Ab- and RNA-positive), the patient returns for one more blood draw and a FibroScan ${ }^{\circledR}$ (hepatic transient elastography) test to obtain a complete diagnosis and assessment of liver function.

Results: Since the HCV Pathway was implemented regionwide in early 2015, total $\mathrm{HCV}$ Ab screening has nearly doubled. HCV Pathway patients are more likely to receive HCV RNA testing (75\% in usual care, $>99 \%$ in $\mathrm{HCV}$ Pathway) and HIV coinfection testing (68\% in usual care, $>99 \%$ in HCV Pathway).

Conclusion: Specific clinical laboratory and regional laboratory protocols support success in screening more than 84,000 patients through the new HCV Pathway.

Keywords: health promotion, prevention, screening, chronic disease, ambulatory/outpatient care, dissemination, implementation, innovation

\section{P12.03 \\ Comparing the Effectiveness of Automated and Live Reminders for a Direct-Mail Fecal Testing Program}

Jennifer Rivelli, ${ }^{1}$ Gloria Coronado, ${ }^{1}$ Morgan Fuoco, ${ }^{1}$ Victoria Gawlik, ${ }^{2}$ Amanda Petrik, ${ }^{1}$ Ricardo Jimenez ${ }^{2}$

${ }^{1}$ Kaiser Permanente Center for Health Research, Portland, OR; ${ }^{2}$ Sea Mar Community Health Centers, Seattle, WA

Background: Patient reminders can improve rates of colorectal cancer screening. As part of the Strategies and Opportunities to STOP Colon Cancer in Priority Populations 
(STOP CRC) study, we partnered with Sea Mar Community Health Centers to conduct two pilot studies on patient reminders. Our first pilot compared the effectiveness of reminder letters, automated phone calls, live phone calls, and text messages in reminding patients to complete a fecal test as part of a mailed fecal test program. Findings showed that, for Spanish-speaking patients, the combined automated/live call (61\%) approach (up to 4 reminders) resulted in a higher fecal test return rate than automated calls $(30 \%)$ alone (which delivered 2 reminders). It is unknown whether the return rate difference is attributable to the relatively high number of phone calls or to the personal communication in the live call scenario. The second pilot compared the effectiveness of automated phones calls only $(\mathrm{n}=6)$ vs combined automated $(n=3)$ and live $(n=3)$ calls, making the total number of call attempts the same for both groups.

Methods: Pilot 2 involved two clinics of Sea Mar, a community-based organization in Washington state. Adults age $50-75$ years who had a primary care visit in the previous year and were not up-to-date with colorectal cancer screening received an informational letter and fecal immunochemical test (FIT) kit by mail. Patients who did not return their FIT kits within 3 weeks were randomized to receive 1) 6 automated phone calls, or 2) 3 automated and 3 live phone calls. We recorded rates of screening completion and rates of patients reached by reminder.

Results: Overall, a total of 271 patients were randomized (72\% who preferred English and 20\% who preferred Spanish). Among the randomized sample, $80 \%$ were enrolled in Medicaid or Medicare, and 56\% reported an annual household income of less than $\$ 20,000$. Analysis is still underway to determine reach. Final results will be presented in the poster.

Conclusion: To date, in the community health center setting, automated and live phone call reminders to a directmail fecal testing program substantially increase colorectal cancer screening rates.

Keywords: cancer, health promotion, prevention, screening, dissemination, implementation, innovation

\section{P12.04 \\ Developing Implementation Strategies With Stakeholders to Promote Firearm Safety as a Suicide Prevention Strategy in Pediatric Primary Care}

Rinad Beidas, ${ }^{1}$ Courtney Benjamin Wolk, ${ }^{1}$ Shari JagerHyman, ${ }^{1}$ Brian K. Ahmedani, ${ }^{2}$ John Zeber, ${ }^{3}$ Joel Fein, ${ }^{4}$ Greg Brown, ${ }^{1}$ Courtney Gregor, ${ }^{1}$ Adina Lieberman, ${ }^{1}$ Steven Marcus ${ }^{1}$

${ }^{1}$ University of Pennsylvania, Philadelphia, PA; ${ }^{2}$ Henry Ford Health System, Detroit, MI; ${ }^{3}$ Baylor Scott \& White Health, Temple, TX; ${ }^{4}$ Children's Hospital of Philadelphia, Philadelphia, PA
Background: The number of youth suicides by firearm has steadily increased in the last decade, representing a public health crisis. Promoting safe firearm storage as a universal suicide prevention strategy in pediatric primary care is one way to address this crisis. Safety Check is an evidence-based practice for improving parental firearm safety behavior in pediatric primary care. This study, Adolescent Suicide Prevention In Routine clinical Encounters (ASPIRE), aims to engender a better understanding of how to implement components of Safety Check as a suicide prevention strategy in pediatric primary care from a system-level perspective.

Methods: We are collaboratively developing implementation strategies in partnership with stakeholders from 2 sites in the National Institute of Mental Health-funded Mental Health Research Network. We surveyed providers and leadership of 82 pediatric primary care practices to understand acceptability and use of the 3 firearm components of Safety Check (ie, screening, brief counseling on firearm safety, provision of firearm locks). In collaboration with stakeholders, we are using intervention mapping and the Consolidated Framework for Implementation Research to systematically develop and evaluate a multilevel menu of implementation strategies for promoting firearm safety as a suicide prevention strategy in pediatric primary care.

Results: Results from the survey suggest that primary care physicians $(\mathrm{N}=103)$ and clinical leaders $(\mathrm{N}=40)$ endorse the acceptability of 2 intervention components, screening and counseling, but reported only sometimes using these strategies. Results indicate neutral acceptability for provision of firearm locks, and physicians report they rarely distribute such devices in pediatric primary care. Qualitative interviews with 9 stakeholder groups are ongoing, as is development of implementation strategies.

Conclusion: This study provides important insights into acceptability and current use of evidence-based practices for safe firearm practices in pediatric primary care for suicide prevention. We also will outline our approach to collaboratively developing implementation strategies with stakeholders across 2 large systems using a systematic and mixed-methods approach.

Keywords: child and adolescent health, patient-provider communication, behavioral and mental health, engagement of stakeholders, clinical decision-making, dissemination, implementation, innovation

\section{P12.05}

Spreading CM-SHARE to Multiple Settings: Strategies, Learnings, and Future Applications

Hannah Husby, ${ }^{1}$ Lily (Shuting) Liang, ${ }^{1}$ Jacob DelatorreReimer, ${ }^{1}$ Cory Mosser, ${ }^{1}$ Xiaowei Yan, ${ }^{1}$ J.B. Jones ${ }^{1}$

${ }^{I}$ Sutter Health, Walnut Creek, CA

Background: Spreading technology applications to new settings requires a thorough understanding of the new 
users, contexts, and spread strategies adapted to the new audiences. It also is important to keep track of variations in implementation that may affect and help explain differences in adoption and use. CM-SHARE is a web-based application that was designed to help manage patients with cardiometabolic conditions at the point of care. In the pilot phase, CM-SHARE was developed with 6 primary care physicians (PCPs) at 2 sites and evaluated using quantitative (application usage) and qualitative (user feedback) measures to accelerate learning and iterative improvement of the application. Herein, we share findings and learnings from efforts to spread CM-SHARE to new users naïve to the product development cycle and in various new settings.

Methods: Eight pharmacists, nurse case managers, and social workers from a case management and care coordination program, 13 certified diabetes educators, and an additional 20 PCPs were given CM-SHARE in a health system in Northern California. Prior to giving the application to the new users, the study team tested different strategies and ways of introducing the tool to new users, creating training packages and setting up individual or teambased meetings to demo the application and understand new settings, workflows, and challenges. Users were asked to help determine the appropriate level of engagement. Once users had the application, application usage was tracked and user feedback was collected.

Results: Of the 20 PCPs who were given the application, 5 used it multiple times during initial roll-out and gave feedback to help understand why CM-SHARE was used and how. Providers outside of primary care gave insight on which features were valuable to them and how the application could be spread to their colleagues. The new settings varied in leadership structure, priorities, and workflows.

Conclusion: Being both deliberate in what data is collected and open with the feedback process helps promote buyin from users and elicits meaningful information that can lead to a better understanding of the utility of application in multiple contexts, improve and identify features with shared value, and help message that value to others.

Keywords: information technology adoption and diffusion, primary care, evaluation research, health care organization, dissemination, implementation, innovation

\section{P12.06}

\section{Secret Sauce to Integrating Technology Innovations Into Care Delivery: An Illustration From CM-SHARE}

\author{
Jacob Delatorre-Reimer, ${ }^{1}$ Lily (Shuting) Liang, ${ }^{1}$ Hannah \\ Husby, ${ }^{1}$ Cory Mosser, ${ }^{1}$ J.B. Jones ${ }^{1}$ \\ ${ }^{1}$ Sutter Health, Walnut Creek, CA
}

Background: Integrating new technology innovations into routine care delivery is challenging; many applications fail to get meaningful user uptake or sustained usage. With the rising demand for new technologies in health care, a thorough understanding of factors and replicable approaches leading to successful integration is needed. This presentation aims to fill this gap by presenting the "secret sauce" that led to successful integration of CM-SHARE into primary care. CM-SHARE is a web-based application that helps providers and patients manage cardiometabolic conditions such as diabetes, hypertension, and dyslipidemia. CM-SHARE has been integrated into two primary care clinics in Northern California and is used by 25+ physicians, case managers, and diabetes educators.

Methods: A user-centered design and development process was employed. Observation of physician's electronic health records (EHR) use and physician-patient interactions during an office visit were conducted to understand the workflows and needs. One-on-one interviews and group design sessions with physicians and patients were also done to design the solution. We built a technology platform that allows CM-SHARE to be seamlessly integrated with the EHR and synthesize data from outside of the EHR. During pilots, web log data were used to track the "who, what, where, when" of each user interaction with the application. Additional interviews were conducted periodically with pilot users to further enhance the application. Results: The user-centered design process yielded a display that gives clinicians a comprehensive picture of the patient's critical health information without any clicks. A live demo of CM-SHARE and the technology that supports its integration with the EHR will be presented. To date, CM-SHARE has been used by 25 users for 7900 encounters with 3712 patients. The most used features include medication adherence, cardiometabolic graphs, and risk calculators, which allow providers to discuss with patients their 10-year risks for heart diseases, kidney failure, vision loss, and amputation.

Conclusion: Intimate involvement of users in the design and development process will help make the technology more relevant and useful, and therefore overcome the adoption gap. Rapid learning and feedback to product development and operating within real-world constraints are key to adoption and sustained use of technology solutions in primary care delivery.

Keywords: information technology adoption and diffusion, primary care, ambulatory/outpatient care, diabetes, chronic disease, dissemination, implementation, innovation, quality improvement, patient experience/satisfaction

\section{MATERNAL AND PERINATAL CARE}

P13.01

Initial Results From a New Algorithm to Identify Pregnancy Outcome Episodes Across Kaiser Permanente Regions

\author{
Sharon Fuller, ${ }^{1}$ Don Bachman, ${ }^{2}$ Sanchita Sengupta ${ }^{2}$
}

${ }^{1}$ Kaiser Permanente Washington Health Research Institute, Seattle, WA; ${ }^{2}$ Kaiser Permanente Center for Health Research, Portland, OR 
Background: The Kaiser Permanente (KP) Center for Effectiveness and Safety Research (CESR) is a national research collaborative comprising KP's research centers in Colorado, Georgia, Hawaii, Mid-Atlantic States, Northern California, Northwest, Southern California and Washington. The CESR Data Coordinating Center (DCC) has built and distributed an algorithm to create a virtual data warehouse (VDW) pregnancy outcome episodes table, containing one record for each identified pregnancy for which an outcome exists.

Methods: The DCC convened a workgroup of stakeholders from all KP regions, including researchers, clinicians, and programmers. The DCC drew on workgroup clinical expertise and case review and from existing pregnancy project and specific CESR site algorithms. The final product is a sophisticated algorithm to identify pregnancy outcomes using a broad range of data sources, extensive diagnosis and procedure code lookup tables, and detailed decision rules. Data sources include health records of pregnancy history, episodes, and deliveries; diagnoses, procedures, and medications from the existing VDW; and mom-baby link tables. Variables include pregnancy outcome type (live birth, spontaneous abortion, therapeutic abortion, stillbirth, ectopic pregnancy, molar pregnancy, and uncertain outcome); outcome procedure (vaginal delivery, cesarean, etc); outcome date; onset date (imputed if not known), estimated date of delivery, last menstrual period, and gestational age; maternal age and gravida/para status; flags for enrollment and utilization during pregnancy; and flags for weak or conflicting evidence and overlapping episodes. Additional maternal information can be obtained from existing VDW demographic and enrollment tables.

Results: Using the multisite program that implements the algorithm, the $8 \mathrm{KP}$ regions have identified a total of more than 3 million pregnancy episodes, with records for most regions dating back to the early 1990s. This poster presents descriptive data, illustrating volumes and trends in outcome types over time and by region. Data were obtained from a regularly scheduled quality assurance program run by each region.

Conclusion: With its large number of records, broad time range, and national reach, we expect the CESR pregnancy outcome episodes table to greatly facilitate single- and multisite research on pregnancy, reproductive health, and preconception health.

Keywords: virtual data warehouse

\section{P13.02}

\section{Mother-Infant Linkage Data for Research Purposes}

Jingyi Zhu, ${ }^{1}$ Avalow Olsen, ${ }^{1}$ Terese A. DeFor, ${ }^{1}$ Amy L. Butani, ${ }^{1}$ Gabriela Vazquez-Benitez ${ }^{1}$

\section{${ }^{1}$ HealthPartners Institute, Bloomington, MN}

Background: HealthPartners Institute has participated in several research networks and studies requiring motherinfant linkages. Importance for this linkage in research includes pregnancy safety and effectiveness studies as well as for infant studies. Study teams have developed their own algorithm or shared-network algorithms targeted to their population of interest. We present an overall view of targeted populations, methods, and preliminary data to guide a wide institutional approach to developing a mother-infant linkage that can be incorporated into the virtual data warehouse.

Methods: Data sources for mother-infant linkage include demographics, utilization, contract numbers, and state birth records. Algorithms selected correspond to the target population. Linkage of mothers with live birth delivery data and health plan membership only may be linked by contract number; mothers and infants receiving care in our institution may be linked using the electronic health record (Epic) table. Mothers and infant may be linked through state birth records. Validity of each method may need to be evaluated and a common hierarchy applied. Preliminary data are available from current efforts: Medication Exposure in Pregnancy Risk Evaluation Program (MEPREP), Vaccine Safety Datalink (VSD), Patient Outcomes Research To Advance Learning (PORTAL), and Birth Defect Study to Evaluate Pregnancy exposureS (BD-STEPS).

Results: Of 115,450 MEPREP women, 152,800 deliveries were identified through procedure and diagnoses codes. An $81 \%$ mother-infant match was reached using Epic table, contract data, and other demographic data combined with hospital admit, discharge, and birth dates. Of 122,184 member-patient infants in the VSD files, $60 \%(n=72,961)$ were linked to member-patient women using state birth data, contract data, and Epic table. Of 108,869 deliveries, $64 \%(\mathrm{n}=69,375)$ had a mother-infant match. Using similar methods as MEPREP, the PORTAL and BD-STEPS studies found a $75 \%$ and $60 \%$ mother-infant match, respectively.

Conclusion: Differences in matching rates may be associated with different inclusion criteria. Understanding these differences will inform investigators how to select their population. Through numerous study-specific efforts, we have expanded our methods for linking mothers to infants and are easily able to participate in multisite studies that require these data.

Keywords: virtual data warehouse, research findings

P13.03

Building, Validating, and Using the Pregnancy Outcome Episodes Table Available at All Kaiser Permanente Regions

Don Bachman, ${ }^{1}$ Sanchita Sengupta, ${ }^{1}$ Jackie Blank, ${ }^{2}$ Sharon Fuller, ${ }^{3}$ Bruce Folck, ${ }^{4}$ Mark Gray, ${ }^{5}$ Carsie Nyirenda, ${ }^{5}$ Catherine Cleveland, ${ }^{1}$ Joanna Bulkley, ${ }^{1}$ Allison Naleway, ${ }^{1}$ Kimberly Vesco ${ }^{1}$

${ }^{1}$ Kaiser Permanente Center for Health Research, Kaiser Permanente Northwest, Portland, OR; ${ }^{2}$ Mid-Atlantic Permanente Research Institute, Kaiser Permanente MidAtlantic States, Rockville, MD; ${ }^{3}$ Kaiser Permanente 
Washington Health Research Institute, Kaiser Permanente Washington, Seattle, WA; ${ }^{4}$ Kaiser Permanente Northern California Division of Research, Oakland, CA; ${ }^{5}$ Kaiser Permanente Institute for Health Research, Kaiser Permanente Colorado, Denver, $\mathrm{CO}$

Background: The Kaiser Permanente (KP) Center for Effectiveness and Safety Research (CESR) is a national research collaborative comprising the research centers from the $8 \mathrm{KP}$ regions. The CESR Data Coordinating Center (DCC) devised a comprehensive algorithm synthesizing data from multiple sources to create a virtual data warehouse (VDW) pregnancy outcome episodes table at each KP region.

Methods: The DCC convened a workgroup consisting of investigators, obstetrics/gynecology providers, pregnancy data experts, and programmers, with representatives from each region, and created criteria for a VDW table containing all pregnancy outcome episodes, available for any research project, and synthesized the best available source data from the electronic medical record (including the Epic Stork module), VDW, CESR, and local data sources. This workgroup evaluated 11 existing algorithms. As none completely met the criteria, the workgroup designed specifications and the DCC created a new algorithm informed by the best methods learned during the evaluation. The table contains outcome type (live birth, stillbirth, spontaneous abortion [SAB], therapeutic abortion [TAB], ectopic, molar, etc); pregnancy outcome and onset dates (imputed if not known); estimated delivery date, last menstrual period, and gestational age; delivery type (vaginal delivery, cesarean, etc); maternal age; gravidity and parity; and variables identifying imputed values, weak data, conflicting evidence, or overlapping episodes. More than 500 episodes were reviewed at the DCC site during the development of the algorithm. More than 200 episodes also were reviewed at 3 other KP regions. Furthermore, the table was validated against pregnancy data from other projects (ie, Vaccine Safety Datalink, Maternal Morbidity, Kaiser Permanente Mid-Atlantic States finance delivery table). The CESR algorithm was continually improved based on these evaluations.

Results: The combined table documents more than 3 million pregnancy episodes (including 2.2 million live births, 12,000 stillbirths, 365,000 SABs, and 300,000 TABs). An independent validation chart review will be reported.

Conclusion: This algorithm produces a comprehensive pregnancy outcome episodes table in the VDW that can facilitate single- and multisite pregnancy and reproductive health research. The algorithm also could be used at other sites with Epic and VDW data. The table has quality flags, source data indicators, and value descriptions so projects can choose all episodes of interest or limit their selection to those with the strongest evidence.

Keywords: virtual data warehouse

\section{MENTAL HEALTH, ALCOHOL AND SUBSTANCE USEIABUSE}

P14.01

Grapheme-Color Synesthesia Is Associated With Posttraumatic Stress Disorder: A Confirmation of Previous Findings and Research Implications

Stuart N. Hoffman, ${ }^{1}$ Thomas G. Urosevich, ${ }^{1} \mathrm{H}$. Lester Kirchner, ${ }^{1}$ Joseph J. Boscarino, ${ }^{2}$ Richard E. Adams, ${ }^{3}$ Charles R. Figley, ${ }^{4}$ Carrie A. Withey, ${ }^{1}$ Ryan J. Dugan, ${ }^{1}$ Joseph A. Boscarino ${ }^{1}$

${ }^{1}$ Geisinger Clinic, Danville, PA; ${ }^{2}$ William James College, Newton, MA; ${ }^{3}$ Kent State University, Kent, OH; ${ }^{4}$ Tulane University, New Orleans, LA

Background: Previously, we reported an association between posttraumatic stress disorder (PTSD) and synesthesia among deployed U.S. veterans (Psychosom Med. 2012;74:912915). Grapheme-color synesthesia is a perception in which a particular letter or number elicits a concurrent image or perception of a specific color. PTSD has been associated with specific neuropsychological processes, including memory function, intelligence level, cognitive processing, and mixed handedness, among other phenomena.

Methods: Since our earlier study reported an association between PTSD and synesthesia, our current objectives were to validate this association using a new study and to explore reasons for this association. To assess this, we randomly surveyed 1730 veterans who were outpatients at Geisinger Health, the largest multihospital system located in central and northeastern Pennsylvania. As in our previous study, all veterans had served in 1 or more warzone deployments.

Results: In our sample, the mean age of veterans was 59.6 years and the prevalence of PTSD was $7.6 \%(95 \% \mathrm{CI}$ : 6.5-9.0). Altogether, 3.4\% of veterans (95\% CI: 2.7-4.4) screened positive for grapheme-color synesthesia. Bivariate analyses suggested that synesthesia was associated with current PTSD (odds ratio: 4.1, $\mathrm{P}<0.001$ ). Multivariable regression results, adjusting for age, gender, marital status, education level, psychotropic medication use, and concussion history, confirmed this association (odds ratio: $3.0, \mathrm{P}=0.006$ ). Synesthesia also was significantly associated with higher anxiety, neuroticism, and attention deficit disorder symptoms $(\mathrm{P}<0.001$ for each).

Conclusion: Our study confirms that synesthesia is associated with PTSD among a second cohort of U.S. veterans. Additional research is recommended to expand these findings and to determine if synesthesia is also a risk factor for PTSD among nonveterans. (Study funding: support for this study was provided in part by U.S. Department of Defense [Contract No. W81XWH-15-1-0506] to Joseph A. Boscarino.)

Keywords: survey research and methods, VA health system, behavioral and mental health, research findings 
P14.02

Male-Female Differences in Diagnosed Mental Health Conditions and Risk of Suicide Mortality

Hsueh-Han Yeh, ${ }^{1}$ Joslyn Westphal, ${ }^{1}$ Yong Hu, ${ }^{1}$ Edward Peterson, ${ }^{1}$ L. Keoki Williams, ${ }^{1}$ Deepak Prabhakar, ${ }^{1}$ Cathrine Frank, ${ }^{1}$ Kirsti Autio, ${ }^{1}$ Farah Elsiss, ${ }^{1}$ Gregory E. Simon, ${ }^{2}$ Arne Beck, ${ }^{3}$ Frances Lynch, ${ }^{4}$ Rebecca Rossom, ${ }^{5}$ Christine Lu, ${ }^{6}$ Ashli Owen-Smith, ${ }^{7}$ Beth Waitzfelder, ${ }^{8}$ Brian K. Ahmedani ${ }^{1}$

${ }^{1}$ Henry Ford Health System, Detroit, MI; ${ }^{2}$ Kaiser Permanente Washington, Seattle, WA; ${ }^{3}$ Kaiser Permanente Colorado, Denver, CO; ${ }^{4}$ Kaiser Permanente Northwest, Portland, OR; ${ }^{5}$ HealthPartners, Bloomington, $M N ;{ }^{6}$ Harvard Medical School and Harvard Pilgrim Health Care Institute, Boston, MA; ${ }^{7}$ Georgia State University / Kaiser Permanente Georgia, Atlanta, GA; ${ }^{8}$ Kaiser Permanente Hawaii, Honolulu, HI

Background: Suicide is a major public health issue in the United States. Although mental health conditions are widely considered to be risk factors for suicide, there is limited data available within the general U.S. population. This study aims to fill this gap as well as assess whether risk differs by sex, given that there are wide variations in both rates of suicide death and health care service utilization between men and women.

Methods: The study sample was obtained from 8 health care systems in the Mental Health Research Network. This study used a case-control study design. Cases were identified as those who died by suicide between 2000 and 2013. Each case was matched with 100 controls from the same health care system and enrolled during the same time period. A total of 2674 individuals who died by suicide as well as 267,400 matched controls were identified. Mental health diagnoses in the year before suicide were extracted from the virtual data warehouse. Conditional logistic regression was used to investigate 5 mental health diagnoses and suicide mortality. Results: Nearly half of cases had at least one mental health diagnosis in the year before death, compared to $13 \%$ for controls. Cases had an average of 5.2 health care visits that included a mental health diagnosis in a year prior to their death, compared to 0.6 visits for controls. Risk of suicide mortality risk was highest among individuals who had a diagnosis of schizophrenia spectrum disorder (adjusted odds ratio [aOR]: 15), followed by bipolar disorders (aOR: 13.2), depressive disorders (aOR: 7.2), anxiety disorders (aOR: 5.8), and attention deficit hyperactivity disorder (aOR: 2.4). After stratification by sex, the risk of suicide among women with a bipolar disorder diagnosis (aOR: 22.9, 95\% CI: $17.8-$ 29.4) was significantly higher than in men (aOR: $12.6,95 \%$ CI: 10.1-15.8).

Conclusion: Nearly all of the mental health diagnoses were associated with increased risk of suicide. The findings will help health care professionals to identify at-risk individuals for suicide and implement effective prevention strategies for both male and female. However, nearly half of individuals who died by suicide did not have mental health diagnoses in the year before death. Therefore, suicide prevention efforts may need to reach beyond those with mental health diagnoses.

Keywords: behavioral and mental health, gender/sex differences, health care, epidemiology

\section{P14.03}

\section{Examining How Health Service Utilization Patterns Vary Among Individuals Before Suicide}

Farah Elsiss, ${ }^{1}$ Kirsti Autio, ${ }^{1}$ Joslyn Westphal, ${ }^{1}$ HsuehHan Yeh, ${ }^{1}$ Yong Hu, ${ }^{1}$ Edward Peterson, ${ }^{1}$ L. Keoki Williams, ${ }^{1}$ Deepak Prabhakar, ${ }^{1}$ Cathrine Frank, ${ }^{1}$ Gregory E. Simon, ${ }^{2}$ Christine Lu, ${ }^{3}$ Frances Lynch, ${ }^{4}$ Rebecca Rossom, ${ }^{5}$ Ashli Owen-Smith, ${ }^{6}$ Arne Beck, ${ }^{7}$ Beth Waitzfelder, ${ }^{8}$ Brian K. Ahmedani ${ }^{1}$

${ }^{1}$ Henry Ford Health System, Detroit, MI; ${ }^{2}$ Kaiser Permanente Washington, Seattle, WA; ${ }^{3}$ Harvard Medical School and Harvard Pilgrim Health Care Institute, Boston, MA; ${ }^{4}$ Kaiser Permanente Northwest, Portland, OR; ${ }^{5}$ HealthPartners Institute, Minneapolis, MN; ${ }^{6}$ Georgia State University / Kaiser Permanente Georgia, Atlanta, GA; ${ }^{7}$ Kaiser Permanente Colorado, Denver, CO; ${ }^{8}$ Kaiser Permanente Hawaii, Honolulu, HI

Background: Health care settings have been identified as strategic environments for implementing suicide prevention approaches. Although health care utilization is common among individuals in the year prior to suicide, there is limited research on how visit patterns differ from those who do not die. This study examined the type and timing of health care visits utilized prior to index date for individuals who died by suicide and matched controls in a U.S. general health care population sample.

Methods: Health care utilization data were collected from 8 health care systems within the Mental Health Research Network using a virtual data warehouse. A total of 2674 individuals who died by suicide between 2000 and 2013 were matched with 267,400 controls by year and location. Visit types, stratified by mental health and chemical dependency diagnoses, within 1 year and within 4 weeks prior to index date were identified. Index date was defined as the date of death for those who died by suicide and the same date for controls.

Results: Most individuals received health services in the year prior to index date ( $92 \%$ of cases vs $86 \%$ of controls); however, in the 4 weeks prior to index date, $57 \%$ of cases received services compared to $29 \%$ of controls. When compared with controls, individuals who died by suicide were more likely to have had a medical visit for a mental health condition ( $54 \%$ vs $15 \%$ within 1 year; $28 \%$ vs $3 \%$ within 4 weeks) or a visit for chemical dependency (28\% 
vs $5 \%$ within 1 year; $10 \%$ vs $1 \%$ within 4 weeks) before the index date. A greater number of cases had inpatient and emergency mental health and chemical dependency visits than controls in both time periods $(\mathrm{P}<0.001)$. Emergency room visits with a mental health diagnosis in the 4 weeks prior to index date were associated with the greatest odds of suicide (adjusted odds ratio: 64.36).

Conclusion: This study provides insight into how health services sought by individuals who died by suicide up to 1 year before their death differed from individuals who did not die by suicide. This may guide prevention efforts toward targeted resource allocation.

Keywords: health promotion, prevention, screening, behavioral and mental health, epidemiology

\section{P14.04}

\section{Successful Implementation of a Multidisciplinary Violence Risk Assessment Tool}

\section{Alexia Terwilliger, ${ }^{1}$ Gayle Godfrey, ${ }^{1}$ Laura Billstein, ${ }^{1}$ Sandi Wewerka ${ }^{1}$}

\section{${ }^{1}$ Regions Hospital, Saint Paul, MN}

Background: Health care professionals are faced with many challenges. One of these challenges is maintaining personal safety at work. In December 2014, Regions Hospital formed a Workplace Violence Committee responsible for the research and development of a violence risk assessment tool currently used throughout the hospital. The primary objective of this study was to determine if the use of the MIAHTAPS violence risk assessment and appropriate interventions reduced the frequency of injuries at Regions Hospital after implementation. MIAHTAPS was designed from two well-known evidence-based tools that have proved to be valid and reliable in different hospital settings.

Methods: A retrospective chart review was completed on all admitted patients to Regions Hospital during the time nursing units were piloting the MIAHTAPS violence risk assessment. A second chart review was completed on the same nursing units after staff were trained and the MIAHTAPS violence risk assessment became mandatory hospitalwide. Security reports were analyzed during both time periods to determine if the implementation of the MIAHTAPS violence risk assessment decreased the number of reported violent incidences and injuries.

Results: The primary aim of this study was to determine if implementation of the MIAHTAPS violence risk assessment tool and appropriate interventions reduce the frequency of violent behavior in 5 specific units at Regions Hospital after implementation. In the pilot time period, there were 69 incidences of reported aggressive behavior and 48 incidences resulting in injury to staff. In the hospitalwide time period, there were 58 reported incidences of aggressive behavior and 28 reported injuries to staff. A Fisher's exact test was performed to test for statistical significance. There was no statistical difference in the number of physical aggressive incidents by time period $(\mathrm{P}=0.15)$. There was a statistically significant decrease in the amount of reported physical injuries to staff $(\mathrm{P}<0.04)$.

Conclusion: Before the implementation of MIAHTAPS, there was no standard of care, such as suggested interventions, for deescalating potentially violent patient behaviors. This study ultimately showed that the incidences of violent behavior did not decrease between the two time periods, but the injuries to staff decreased significantly.

Keywords: human subjects research, aging, elderly, geriatrics, health care organization, workforce, nursing, acute inpatient care, behavioral and mental health, clinical decision-making, hospital, dissemination, implementation, innovation, quality improvement

P14.05

Risk of Suicide in Patients With Traumatic Brain Injury

Rebecca Rossom, ${ }^{1}$ Edward Peterson, ${ }^{2}$ Mansi Sethi Chawa, ${ }^{2}$ Cynthia DiGiandomenico, ${ }^{3}$ Deepak Prabhakar, ${ }^{2}$ Yong Hu, ${ }^{2}$ Ashli Owen-Smith, ${ }^{4}$ Gregory E. Simon, ${ }^{5}$ L. Keoki Williams, ${ }^{2}$ Samuel Hubley, ${ }^{6}$ Frances Lynch, ${ }^{7}$ Arne Beck, ${ }^{8}$ Beth Waitzfelder, ${ }^{9}$ Christine Lu, ${ }^{10}$ Brian K. Ahmedani ${ }^{2}$

${ }^{1}$ HealthPartners Institute, Minneapolis, MN; ${ }^{2}$ Henry Ford Health System, Detroit, MI; ${ }^{3}$ Regions Hospital, Minneapolis, MN; ${ }^{4}$ Kaiser Permanente Georgia, Atlanta, GA; ${ }^{5}$ Kaiser Permanente Washington, Seattle, WA; ${ }^{6}$ University of Colorado Denver, Denver, CO; ${ }^{7}$ Kaiser Permanente Northwest, Portland, OR; ${ }^{8}$ Kaiser Permanente Colorado, Denver, CO; ${ }^{9}$ Kaiser Permanente Hawaii, Honolulu, HI; ${ }^{10}$ Harvard Medical School and Harvard Pilgrim Health Care Institute, Boston, $M A$

Background: Traumatic brain injury (TBI) increases the risk of suicide by nearly 9 -fold, greater than other comorbid illnesses. Our objective was to determine what further increases suicide risk in people with TBI to better guide future potential interventions

Methods: Design: This was a case-control study. Setting: 8 Mental Health Research Network-affiliated health care systems that serve over 5 million patients across the United States were included. Electronic health record, insurance claim, and other administrative data as well as state mortality records were organized into a virtual data warehouse (VDW). Participants: 61 cases with TBI who died by suicide and their 75 matched controls with TBI who did not die by suicide between January 1, 2000, and December 31, 2013, were included. Main outcome measures: Suicide deaths were identified via the VDW using ICD-10 codes of X60-X84 and Y87.0. Analysis: Descriptive statistics characterized the sample stratified by cases and controls, while conditional logistic regression models estimated the 
adjusted odds (aOR) of suicide.

Results: Men (aOR: 5.00, 95\% CI: 1.33-1.67, $\mathrm{P}=0.016$ ) and people with psychiatric diagnoses in general (aOR: 5.96, 95\% CI: $1.35-26.26, \mathrm{P}=0.02$ ) or depression specifically (aOR: 4.86, 95\% CI: 1.20-19.64, $\mathrm{P}=0.03$ ) were at greatly increased risk of suicide beyond the already high baseline rate for people with TBI. Risk for suicide in people with TBI generally increased with age (aOR: 1.04 per year, 95\% CI: $1.00-1.08, \mathrm{P}=0.047)$. There were no significant associations with anxiety, alcohol, or tobacco use disorders.

Conclusion: Our findings provide clinicians with important predictors of suicide for people with TBI, a population that should be carefully screened for suicide risk.

Keywords: behavioral and mental health

\section{METHODS, DESIGNS, ANALYTICS TOOLS}

\section{P15.01 \\ Innovative Methods to Build and Assess Virtual Data Warehouse Mom-Baby Linkage Across All Kaiser Regions}

Sanchita Sengupta, ${ }^{1}$ Don Bachman, ${ }^{1}$ Jackie Blank, ${ }^{1}$ Carmen Wong, ${ }^{1}$ Mark Gray, ${ }^{1}$ Catherine Cleveland ${ }^{1}$

\section{${ }^{1}$ Kaiser Permanente, Portland, OR}

Background: The Kaiser Permanente Center for Effectiveness and Safety Research (CESR) is a national research collaborative comprising KP's research centers in 8 regions: Colorado, Georgia, Hawaii, Mid-Atlantic States, Northern California, Northwest, Southern California, and Washington. The Data Coordinating Center (DCC) of CESR built an extract, transform, load (ETL) package that creates a virtual data warehouse (VDW) pregnancy database based on a common data model at each of the 8 regions. One of the tables in the database is the preg_mom_baby_link, consisting of 6 variables including mother identification number, baby identification number, baby's date of birth, baby's sex, and primary data source. We sought to evaluate the best method to link records while accounting for differences in regions. Identifying linked records was challenging due to the differences among the delivery settings (hospital vs nonKaiser hospital regions) and deliveries occurring outside hospitals, like birth centers, or when the mother was not enrolled in the system. We designed an algorithm that uses the best available data sources from each site.

Methods: We distributed an algorithm-based ETL that runs against the Stork module of Epic/HealthConnect ${ }^{\circledR}$, Health Care Systems Research Network, CESR VDW tables, and prior data from region-specific projects. We used two additional approaches and validated the records against mothers with live-births: 1) identifying mom-child relationship using insurance benefit subscriber group; and 2) identifying family units using location coordinates and subsequently identifying mother-child relationship within the family unit using demographics information. The resulting pregnancy database will be periodically assessed using CESR DCC quality assessment processes. We will provide an analysis of confidence scores based on comparison against known links, maternal enrollment status, and source of data.

Results: The algorithm was validated by regions with existing mom-baby linkage. About 2.5 million mom-baby links were recorded at Kaiser regions, with about $89 \%$ having data on delivery, gestational age, and other key variables. Almost $100 \%$ of the linked records for two nonhospital sites came from the benefits-subscriber group and location coordinates method.

Conclusion: By using all available data sources, this algorithm improved identification of mom-baby links for all Kaiser sites, collectively resulting in the largest database of linked records of mother-child.

Keywords: virtual data warehouse

\section{P15.02 \\ Comparison of Two Methods to Obtain Consent for Patient-Reported Outcomes in a Pragmatic Trial: Maximizing Data Completeness and Participant Autonomy}

Jeanette Y. Ziegenfuss, ${ }^{1}$ Kayla Dean, ${ }^{1}$ Anna Bergdall, ${ }^{1}$ Mary Sue Beran, ${ }^{1}$ Beverly Green, ${ }^{2}$ Alison Helm, ${ }^{1}$ Chris Norton, ${ }^{3}$ Patrick J. O'Connor, ${ }^{1}$ Leif I. Solberg, ${ }^{1}$ Patty Haugen, ${ }^{3}$ JoAnn Sperl-Hillen, ${ }^{1}$ Karen L. Margolis ${ }^{1}$

${ }^{1}$ HealthPartners Institute, Minneapolis, MN; ${ }^{2}$ Kaiser Permanente Washington, Seattle, WA; ${ }^{3}$ unaffiliated

Background: In pragmatic trials, researchers often rely on electronic health record (EHR) data. A waiver of consent may be granted if the research could not be practicably carried out otherwise. However, when EHR data are supplemented with patient-reported outcomes (PROs), obtaining consent is feasible and appropriate. If participants decline consent to use their EHR data, this could reduce data completeness and statistical power. In a cluster-randomized trial to optimize treatment for high blood pressure, the primary outcome is blood pressure obtained from EHR data and secondary outcomes are PROs obtained by survey. The study team, including patient partners, designed a pilot experiment to understand how to maximize both survey and EHR data completeness, while protecting participant autonomy.

Methods: Eligible participants, enrolled in the study under a waiver of consent, were mailed a survey and cover letter that included consent language. We randomized 213 participants to two methods of obtaining consent to use EHR data to compare the effect on survey and EHR data completeness. In the first method, the respondents were asked in the survey for permission to use their EHR data for research. Primary outcome data from the EHR would be unavailable for 
those that responded "no." The second method included a statement in the cover letter that the use of EHR data for research is part of the study and participants consent to this with survey completion. We hypothesized that the second method would reduce survey response rate but that all respondents would have usable EHR data.

Results: Overall response rate was 45\% $(n=96)$ and did not differ between the two consent methods. When respondents were asked for permission to use EHR data, 11\% declined, resulting in a $5 \%$ reduction in the sample available for the blood pressure primary outcome.

Conclusion: The second method of obtaining consent did not reduce survey response rates and maximized the completeness of the EHR outcomes data. The full study will survey 2000 individuals and will include language in the cover letter indicating that by returning the survey, participants consent to using their health records for research. Other changes to improve survey response rates are being tested.

Keywords: human subjects research, program evaluation, survey research and methods, confidentiality/HIPAA

\section{P15.03}

\section{Enhancing Death Data for Multisite Studies With a Bundled Application to National Death Index}

Morgan Fuoco, ${ }^{1}$ Phillip Crawford, ${ }^{1}$ Dea PapajorgjiTaylor, ${ }^{1}$ Reesa Laws, ${ }^{1}$ Mary Ann McBurnie, ${ }^{1}$ Kristina Hansen, ${ }^{2}$ Lisa Weatherby ${ }^{3}$

${ }^{1}$ Kaiser Permanente Center for Health Research, Portland, OR; ${ }^{2}$ Kaiser Permanente Washington Health Research Institute, Seattle, WA; ${ }^{3}$ World Health Information Science Consultants, LLC, Newton, MA

Background: The occurrence of death is an important outcome in many research endeavors. The National Death Index (NDI) is an often-used data source that can be used to identify or confirm death and cause of death. Ensuring that death outcome data are complete, accurate, and standardized is particularly important in a multisite study; however, coordinating the preparation of standardized NDI data requests across sites is challenging. The Data Coordinating Center (DCC) for a large multicenter study of opioid overdose incidence and risk factors among patients using long-term opioid treatment for chronic pain has developed a NDI application approach to support a multisite study examining, among other outcomes, death and cause of death relating to opioid use. This approach allows sites to create data files that are formatted to NDI specifications, are standardized across sites, and maintain participating institutions' protections for personal health information.

Methods: Each of the four participating sites maintains a Sentinel Common Data Model. As part of the application process, sites will provide a list of their data sources, a summary of the project activities at the site level, applicable documentation verifying institutional review board approval, and signed confidentiality agreements to abide by the terms and conditions related to disclosure of individual information. The DCC will distribute code to sites that will identify patients according to criteria developed by study investigators and will create, according to NDI specifications, standardized files containing records of patients for whom death information is needed. Sites will customize a portion of the code to extract patient Social Security number and name, two data elements required by NDI. Each site will submit its own data request in order to maintain local patient confidentiality requirements. The DCC will submit the overall application.

Results: The application process developed by the DCC resulted in an efficient standardized approach for submitting a single NDI application across multiple sites.

Conclusion: Employing a standardized bundled NDI application approach using distributed code and centralized administrative resources will reduce the effort required by the sites and will minimize errors and inconsistencies in NDI data request files across sites.

Keywords: substance abuse and addiction, research administration, contracting, operations, quality improvement

\section{P15.04 \\ Validation of Spontaneous Abortion Following Inadvertent Quadrivalent Human Papillomavirus Vaccination During Pregnancy in the Vaccine Safety Datalink}

Gabriela Vazquez-Benitez, ${ }^{1}$ Elyse O. Kharbanda, ${ }^{1}$ Heather Lipkind, ${ }^{2}$ Sangini Sheth, ${ }^{2}$ Jingyi Zhu, ${ }^{1}$ Allison Naleway, ${ }^{3}$ Nicola Klein, ${ }^{3}$ Rulin Hechter, ${ }^{3}$ Matthew Daley, ${ }^{3}$ Jim Donahue, ${ }^{4}$ Mike Jackson, ${ }^{5}$ Alison Kawai, ${ }^{6}$ Zhiyuan Xu, ${ }^{1}$ Sophia Newcomer, ${ }^{3}$ James D. Nordin ${ }^{1}$

${ }^{1}$ HealthPartners Institute, Bloomington, $M N ;{ }^{2}$ Yale School of Medicine, New Haven, CT; ${ }^{3}$ Kaiser Permanente Northwest, Portland, OR; ${ }^{4}$ Marshfield Clinic Research Institute, Marshfield, WI; ${ }^{5}$ Kaiser Permanente Washington Health Research Institute, Seattle, WA; ${ }^{6}$ Harvard Pilgrim Health Care Institute, Boston, MA

Background: The Vaccine Safety Datalink maintains a pregnancy episode table to evaluate the safety of maternal vaccination. It uses a pregnancy episode algorithm (PEA) to retrospectively identify pregnancies from electronic health and birth data. Spontaneous abortions (SAB) are identified through diagnosis and procedure codes and assigned a default gestational age (GA) of 10 weeks. Since outcome misclassification could cause bias, our objective was to validate $\mathrm{SAB}$ and GA within a cohort study of inadvertent human papillomavirus vaccine (4vHPV) administration during pregnancy.

Methods: The cohort was comprised of women 12-27 years potentially exposed to $4 \mathrm{vHPV}$ during three exposure 
windows: distal (16-22 weeks prior to last menstrual period [LMP]), peripregnancy (the 42 days prior to LMP), and during pregnancy (up to 19 completed weeks gestation). Seven sites conducted structured chart reviews for SAB, identified exclusions, and initial LMP dating. Test-retest validity was assessed on $10 \%$. Case adjudication was performed by two obstetricians for eligible pregnancies. Adjudicators recorded the range of fetal demise dates and GA at which fetal demise could have occurred. Validation was performed for SAB outcomes, dating of fetal demise, and GA at fetal demise.

Results: From a cohort of 3695 pregnancies, 843 (23\%) potential SAB were identified by the PEA. Kappa was above $70 \%$ for SAB outcome, GA documentation, and ultrasound documentation. A total of $308 \mathrm{SAB}(37 \%)$ were adjudicated. In total, $66 \%$ of episodes were confirmed as a SAB occurring at $\geq 6$ weeks gestation, $7 \%$ did not have records to confirm the pregnancy outcome, and $21 \%$ had other etiologies such as ectopic or molar pregnancy, pregnancy loss before 6 weeks, or other study exclusions. Based on LMP dating, 29\% were not exposed to 4vHPV; mean GA in weeks was 9 (SD: 3 ). Among those adjudicated, mean recorded range of GA was 2.6 (SD: 2.2) weeks. Fetal death dating departed from PEASAB dating by an average of 0.7 (SD: 1.1) weeks, with a mean recorded range of 1.3 (SD: 2.1) weeks.

Conclusion: In order to study vaccine-SAB associations, chart abstraction and, frequently, adjudication are needed to refine estimates. Uncertainty in dating can be incorporated in sensitivity analyses.

Keywords: epidemiology

P15.05

Highly Motivated Population Provides Two Successful Recruitment Methods to the TMD SelfCare Study and PACT Program

\author{
Elanadora Sour, ${ }^{1}$ Jeanette Y. Ziegenfuss, ${ }^{1}$ Gabriela \\ Vazquez-Benitez, ${ }^{1} \quad$ Elizabeth $\quad$ Grossman, ${ }^{1}$ Robin \\ Whitebird, ${ }^{2}$ James Fricton ${ }^{1}$
}

${ }^{1}$ HealthPartners Institute, Bloomington, MN; ${ }^{2}$ School of Social Work, University of St. Thomas, Saint Paul, MN

Background: Research study recruitment and committed participation is an essential but challenging component of clinical research. In a pilot study testing the feasibility of recruiting and retaining patients into an online self-care program for temporomandibular disorder (TMD) pain management, we tested two recruitment methods.

Methods: Two methods were used to recruit participants. Invitation letters were sent to affiliated patients or members with a recent diagnosis of TMD. Others were recruited through self-referral after exposure to the study by other means, including a provider's recommendation or brochure displayed in a dental clinic. Both methods offered initial telephone screening and referral to an online screening questionnaire. If eligible, patients were randomized to the online Personalized Activated Care and Training (PACT) program or traditional self-care. Participant feedback was obtained from program evaluations at 8 and 16 weeks, unsolicited comments, and qualitative phone interviews from a subset of PACT participants.

Results: Among the 81 randomized participants, 60\% were recruited by letters sent and $40 \%$ by self-referral. Of 150 letters sent, 49 individuals were recruited into the study, and $61 \%$ of these recruits initiated phone or email contact prior to an outbound recruitment call. Of the 47 self-referrals, $68 \%$ of these were successfully recruited into the study. Survey completion rates were $93 \%$ and $91 \%$ at the 8 - and 16 -week follow-up evaluations, respectively. Pain was a common explanation of participant's initial interest and study compliance. Participants expressed, "I've tried everything. I hope this helps [my pain]!" One highly engaged participant reported, "I found it to be a good alternative to avoid having to take pain medication for my jaw."

Conclusion: The TMD Self-Care pilot study demonstrated two successful recruitment approaches that yielded high recruitment and retention rates as exhibited by follow-up survey response rates. Participant feedback suggests that this highly motivated population is seeking alternative methods to control chronic pain. Understanding these successful recruitment rates and the incentive of controlled chronic pain bodes well for a planned larger trial.

Keywords: clinical trials

\section{P15.06}

Iterative Design and Embedding Research Into an Operational Survey of Health and Well-Being

\author{
Jeanette Y. Ziegenfuss, ${ }^{1}$ Casey Easterday, ${ }^{1}$ Jeffrey \\ Anderson, ${ }^{1}$ Jason Gallagher, ${ }^{2}$ Susan Knudson, ${ }^{2}$ \\ Thomas Kottke, ${ }^{2}$ Nico Pronk, ${ }^{2}$ Sachin Rauri ${ }^{2}$
}

${ }^{1}$ HealthPartners Institute, $\quad$ Minneapolis, $\quad M N$; ${ }^{2}$ HealthPartners, Minneapolis, $M N$

Background: To create a summary measure of health and well-being for our organization, we needed a subjective measure of well-being necessarily dependent on self-report. In July 2015, we launched a member survey for this purpose. While there is a secular trend of falling response rates, we have introduced a number of survey implementation changes that have increased response rates to nearly $50 \%$, approximately doubling our initial response rates. In this presentation, we describe the implementation and impact of iterative design changes to the survey in the context of a learning health system, including the observed impact on response rate and potential impact on nonresponse bias.

Methods: In July 2015, we began a monthly survey of a random cross-section of 1448 members. The initial survey process included a mailing of a 7-page survey, followed by a second mailing and phone follow-up to nonresponders. Our 
current process includes an initial email survey to the $40 \%$ of members who have an email address on file, a single mailing of a 3-page survey with a \$2 incentive and subsequent phone follow-up to nonresponders. Most changes have been introduced experimentally, allowing us to understand the impact of each independently.

Results: To date we have collected 14,267 surveys. We have improved our response rate from $26 \%$ to a high of $50 \%$. We have learned the positive impact of a $\$ 2$ incentive compared to no incentive, a shorter or mid-length survey compared to a longer survey, and the null impact of including a picture with an email request to complete a web-based survey. We also will report on the overall impact of introducing an additional mode for survey completion.

Conclusion: In the context of a robust data collection effort to meet organizational needs, we have embedded iterative experiments to improve survey response rates and representativeness of the resultant data.

Keywords: survey research and methods

\section{P15.07}

\section{Methods and Guidelines for Integrity of Multivariable Analysis of Real-World (Observational) Data}

\section{Drew Levy ${ }^{1}$}

\section{${ }^{1}$ GoodScience, Inc., Mountain View, CA}

Background: The credibility of observational data analysis (ODA) for scientific evidence is a prevalent concern. Numerous threats militate against validity, reproducibility, integrity of inference, and the self-correcting mechanism of the scientific method. Sampling error is only one component in a long sequence of distortive forces in the scientific process that informs the clinical knowledge base, and often not the most important. Many methodological and systematic pitfalls introduce loss and distortion of the "signal" representing causal relationships in nature, much as noise compromises transmission in a communication channel. Existing practices (including analysis methods, standards, and guidelines) for multivariable ODA borrow substantially from those for randomized trials and do not specifically address many idiosyncrasies of ODA that must be taken into account if reproducible and scientifically meaningful results are to be obtained. Updated approaches to addressing ODA quality (ie, veridicality) are needed.

Methods: We deconstruct the process of generating scientific evidence about health effects and outcomes with ODA and identify prevalent, but often overlooked, sources of potential distortion of information and inference. Our perspective connects multiple-bias modeling, causal graph theory, modeling strategies, reproducible research methods, and Bayesian epistemology to define a coherent approach to ODA that optimizes inferential integrity. On this basis, we make recommendations for remediation at critical points.

Results: Major points of focus for revised practice standards include a) explicit specification of the underlying causal and modeling assumptions; b) uncertainties due to model specification, overfitting, and predictive optimism; c) formal incorporation of reproducibility and expectation for reproduction of research into ODA; and d) expression and evaluation of results in terms of reduction of uncertainty and alteration of prior belief.

Conclusion: The value of ODA for clinical decisionmaking is constrained by uncertainties above and beyond those represented in estimated standard errors. This theory of a system of practice suggests guidelines for ODA and a standard for ODA evaluation. This model for ODA encourages a coherent approach focused on support for the self-correcting mechanism in science and optimization of inferential integrity.

Keywords: observational study, risk adjustment, clinical outcomes, biostatistics, research findings

P15.08

Does the Point-of-Care Tool Improve Provider
Efficiency? Application of Electronic Health
Record Audit Log Data

Xiaowei Yan, ${ }^{1}$ J.B. Jones, ${ }^{1}$ Jacob Delatorre-Reimer, ${ }^{1}$ Hannah Husby, ${ }^{1}$ Cory Mosser, ${ }^{1}$ Ridhima Nerlekar, ${ }^{1}$ Lily (Shuting) Liang, ${ }^{1}$ Satish Mudiganti, ${ }^{1}$ Zijun Shen, ${ }^{1}$ Walter F. Stewart ${ }^{1}$

\section{${ }^{1}$ Sutter Health, Walnut Creek, CA}

Background: As "apps" that work with electronic health record (EHR) systems become increasingly common, an important question is how to evaluate an app's effectiveness, particularly to answer the question of whether the app can overcome the inertia of habit by improving physician efficiency. We describe an EHR app (CM-SHARE) for cardiometabolic conditions that has been developed to improve physician efficiency and close care gaps (improve evidence-based care). Herein, we describe our approach to measuring the impact on physician efficiency using EHR audit log data and learnings from user feedback.

Methods: Population: CM-SHARE was implemented in 2 primary care clinics for 6 providers since April 2016. By studying audit data, we can reconstruct major steps in the clinic workflow as well as how the EHR is used during each time interval. The workflow measures derived from audit $\log$ file were validated by in-clinic observation of 100 encounters from 4 providers. We used pre-post case-control cohort selection to determine comparable cases (ambulatory visits in which CM-SHARE was used) and controls (ambulatory visits in which CM-SHARE was not used) for the preimplementation and postimplementation periods. Physician EHR time and total number of EHR clicks in the examination room are primary outcomes. We used a mixedeffect model to evaluate the intervention's effect. Patient characteristics (age, gender, level of service, primary reason 
for visit, comorbidities) also were controlled in the model to remove a residual confounding effect.

Results: After patient-level matching, there were 1037 encounters from 290 intervention patients, 1008 encounters from 290 postimplementation matched controls, and 2901 encounters from 870 preimplementation matched controls. Comparing intervention to control patients, there was no difference in overall encounter duration, regardless of encounter characteristics (ie, level of service). CM-SHARE significantly reduced providers EHR time in the exam room by $25 \%-35 \%$ for diabetes- or hypertension-related encounters. The impact of CM-SHARE also was presented through a $21 \%-36 \%$ reduction of provider total number of EHR clicks for diabetes- or hypertension-related encounters. Providers' overall EHR activities were not significantly reduced.

Conclusion: EHR audit log is a helpful tool for evaluating "apps" in improving physician efficiency.

Keywords: cardiovascular disease, information technology adoption and diffusion, primary care, health care organization, technology assessment

\section{PATIENT, CLINICIAN, AND HEALTH SYSTEM ENGAGEMENT}

\section{P16.01}

An Emergency Department Intranet Site Is an Effective Method of Making Time-Sensitive Information Available to Frontline Staff

\section{Jessie Nelson, ${ }^{1}$ Jacqueline Kelly Hegarty ${ }^{1}$ \\ ${ }^{1}$ Emergency Medicine, Regions Hospital, Saint Paul, MN}

Background: Regions Hospital is a Level 1 trauma center and stroke center with an emergency department (ED) serving 80,000 patients per year. More than 450 full- and part-time staff members work in the ED and need to provide care to a wide variety of patients at all hours of the day with continuously evolving guidelines, practice patterns, interdepartmental agreements, quality standards, and regulatory requirements.

Methods: Our department developed a robust intranet site (EDNET) based on the Microsoft Sharepoint ${ }^{\mathrm{TM}}$ platform in 2014 to create a repository for up-to-date information that is readily accessible by ED frontline staff on a 24/7 basis.

Results: Since 2014, EDNET has become the largest department intranet site in Regions Hospital, with 11836900 hits per month in 2017. We've developed direct links to EDNET from our electronic medical record. After brief instruction from information technology services, an emergency medicine physician was able to create the site architecture, optimized for frontline staff. Department leadership staff members with limited website expertise are able to quickly upload pertinent operational information.
EDNET stores text from emailed operational updates, freeing staff from the need to access email during busy shifts. A robust search function allows staff to quickly search for pertinent information. Volume of search queries varies from 145 to 401 unique queries per month in 2017. Most common search terms are "dka," "ortho grid," "tia," "tpa," and "detox." We are able to quickly update information on EDNET without having to tax limited information technology availability. We also can highlight pertinent policies and make them more readily available to staff.

Conclusion: Our ED has found benefit in the use of a Sharepoint-based intranet site as a one-stop shop for the information that our staff needs to safely and effective care for our patients.

Keywords: health care workforce, information technology, hospital, dissemination, implementation, innovation, research findings

P16.02

Assessing Cervical Cancer Patients' Willingness and Ability to Serve as Health Care Educators to Advocate for Human Papillomavirus Vaccine Uptake

Ilene Ladd, ${ }^{1}$ Radhika Gogoi, ${ }^{1}$ Tyler Bogaczyk, ${ }^{2}$ Sharon Larson $^{3}$

${ }^{1}$ Geisinger Health, Danville, PA; ${ }^{2}$ Drexel University, Philadelphia, PA; ${ }^{3}$ Main Line Health / Thomas Jefferson University, Philadelphia, PA

Background: Patients have served as powerful health advocates for prevention of various diseases including lung and breast cancer. This study explores whether cervical cancer patients may serve as health advocates to adolescents and their parents in encouraging the uptake of the human papillomavirus (HPV) vaccine. Despite recommendations that adolescents age 11-12 years receive the vaccine, only about half of females and less than half of males receive the recommended vaccine dose. HPV is the cause of nearly all cervical cancer.

Methods: The study targeted an opportunity sample of women seen in the gynecologic oncology clinic for cervical cancer. During interviews, patients were asked about provider conversations regarding causes or prevention of cervical cancer, what HPV was, what the HPV vaccine was, what discussions they may have had with family or friends about the causes or prevention of cervical cancer, and whether they would be willing to talk with family or friends about the HPV vaccine. A total of 23 interviews were conducted in 2016-2017. Patients ranged from 28 to 85 years of age. Four team members developed a preliminary coding list, refined the list into overarching themes, then used these themes to code the interviews.

Results: Six primary themes resulted from analysis of the transcripts - 1) patients' expressed fears about vaccines; 
2) low level of health literacy and understanding of the causes of cervical cancer; 3) patients' acquiring health information from sources other than medical professionals (eg, television, internet); 4) whether or not providers held conversations with patients regarding HPV and the vaccine; 5) patient stigma or lack of stigma surrounding cervical cancer; and 6) patients' willingness or reluctance to serve as a health care educator.

Conclusion: While cervical cancer patients overall expressed a willingness to serve as health care educators, barriers remain. Low health literacy expressed as vaccine fear and a lack of understanding of the causes of cervical cancer persist. Many patients described receiving their HPV vaccine information from television advertising, as opposed to coming from their health care provider. These issues will need to be addressed in order for cervical cancer patients to be effective health care advocates.

Keywords: human subjects research, cancer, health promotion, prevention, screening, patient-provider communication, qualitative research

\section{P16.03}

Improving Response Rates in Patient-Reported Outcome Measurement Questionnaires in the Orthopedic and Neurosurgery Patient Population

\section{Megan Reams, ${ }^{1}$ Deepika Appana, ${ }^{2}$ Leif I. Solberg, ${ }^{2}$ Vijayakumar Thirumalai, ${ }^{2}$ Curtis Boehm, ${ }^{3}$ Richard Paskach, ${ }^{2}$ Thomas Ahlers ${ }^{3}$}

${ }^{1}$ TRIA Orthopaedic Center, Bloomington, MN; ${ }^{2}$ HealthPartners Institute, Bloomington, MN; ${ }^{3}$ Park Nicollet Clinic, St. Louis Park, MN

Background: The effective and efficient collection of patient-reported outcome data and integration of such data directly into the electronic medical record (EMR) and downstream databases has often been referred to as the holy grail for determining the effectiveness of orthopedic treatment. The manual process of collecting such data has proven costly, ineffective, and disruptive to clinical workflows. Poor baseline and post-surgical follow-up survey response rates are difficult to overcome. This project focused on automating data capture using existing tools to improve response rates, unburden care teams, and promote use of the data for shared decision-making as well as including the data in existing registries.

Methods: The need for patient-reported outcome data for public reporting and to meet payer and governmental programs and internal quality improvement prompted HealthPartners to pursue solutions for collecting patientreported outcome measures (PROM) through pilot within the orthopedic and neurosurgery departments. In a collaborative effort between TRIA Orthopaedic Center, Park Nicollet and HealthPartners orthopedics and neurosurgery, and
HealthPartners Institute, these units agreed on a common system using email with internet links, as well as paper surveys for those without computer access, combined with in-clinic tablets for nonresponders. The system feeds results into our EMR system and clinical workflows for patients having a knee replacement, hip replacement, or spinal discectomy/laminectomy or fusion.

Results: Over the past 5 months, since implementation, we've collected 1033 more outcomes questionnaires from our patients than we did in 2016. Our 1-year response rate has improved from $29 \%$ to $74 \%$. With the automated identification of patients, staff no longer have to manually identify and collect PROM data. Staff also are prompted in the EMR when a patient is due for a follow-up questionnaire. Conclusion: In large specialty care settings, capture of outcomes data is challenging due to cost, workflow, and patient challenges. By incorporating advanced multimodal data capture into clinical workflows and EMR system, response rates at baseline and postsurgery have achieved levels that are credible for stakeholder needs and quality improvement.

Keywords: information technology adoption and diffusion, survey research and methods, patient-provider communication, health care organization, patient-reported outcomes/function

\section{P16.04 \\ Creating a Culture of Learning and Improvement in Dentistry}

\section{Brad Rindal, ${ }^{1}$ Nico Pronk, ${ }^{1}$ David Gesko, ${ }^{2}$ Jeffrey} Ogden, ${ }^{2}$ William Rush ${ }^{1}$

${ }^{1}$ HealthPartners Institute, Bloomington, $M N$; ${ }^{2}$ HealthPartners, Bloomington, $M N$

Background: Health care is being challenged to achieve the Triple Aim as defined by the Institute of Medicine. Learning health systems (LHS) represent a model with much potential to achieve the Triple Aim when embedded in a culture of learning and improvement. While LHS concepts have been well described, the practical steps to create such a system are not well defined. Dentistry faces challenges in establishing the necessary infrastructure to become a learning health system.

Methods: We examined the current LHS literature that defines the framework for measuring progress in creating the infrastructure of a LHS. This framework was utilized to measure progress and consider future steps in creation of a LHS in dentistry. We looked for entities in dentistry who have publically declared their intention to create a LHS.

Results: The current literature provided a consistent framework for a LHS. This literature comes from hospital, health care, and educational settings but none from dental settings. The National Institutes of Health/National Institute 
of Dental and Craniofacial Research-funded National Dental Practice-Based Research Network has created an infrastructure that links many dental practices in the United States and creates a culture of learning and improvement but lacks the knowledge grid and the feedback loops that allow for learning and sharing of knowledge. We identified 3 large dental groups within health care systems that have made progress in building cultures of learning and improving and in the creation of the knowledge grid, and that are working to create a LHS.

Conclusion: Dentistry has made progress in establishing a LHS, although these efforts have been limited to a few organizations. The most progress has been achieved in establishing network for sharing, and the case example of HealthPartners Dental Group will be presented as an organization that has been able to establish a culture of learning and quality improvement. Challenges remain in establishing the knowledge grid and the essential systemic feedback loops. These findings establish important factors that will shape the future agenda for creation of a LHS in dentistry.

Keywords: dental care, quality improvement

\section{P16.05}

Transgender Response and Quality of Care Study at Kaiser Permanente Mid-Atlantic States: Patient Focus Groups

\section{Suma Vupputuri, ${ }^{1}$ Christine Truong, ${ }^{1}$ Ayanna Wells, ${ }^{1}$ Alphonse Derus, ${ }^{1}$ E.W. Emanuel ${ }^{1}$}

\section{${ }^{1}$ Kaiser Permanente Mid-Atlantic States, Rockville, MD}

Background: Transgender (TG) patients are a heterogeneous group of health care consumers and the quality of their care is unknown. The purpose of this study is to describe Kaiser Permanente Mid-Atlantic States (KPMAS) TG care from the patient perspective using qualitative analyses from focus groups.

Methods: A comprehensive literature review was conducted to inform the development of the focus group script. We solicited feedback on experiences with KPMAS health care, barriers/facilitators of care, expectations, and satisfaction. Recruitment consisted of a brief message sent via KP.org to patients in the TG registry. Of the total 254 patients who were messaged, 54 responded by phone and/or email to participate. We recruited 39 patients to participate in 1 of 4 focus groups, and a total of 28 patients attended the 2-3-hour group discussions. (Groups ranged from 6 to 8 patients). Atlas.ti software was used to code and organize themes for the typological qualitative analysis.

Results: Of the $28 \mathrm{TG}$ patients who participated in the focus groups, 11 identified as female, 3 as male, 6 as transwomen, 1 as transman, 5 as nonbinary/gender queer, and 2 as not sure/questioning or other. Focus group participants were more likely to be white $(68 \%)$, have greater than a high school education $(89 \%)$, be hormone users $(89 \%)$, and have no history of TG surgical procedures (64\%). The most common themes coded in preliminary analyses were confidence in providers' care ("It's insulting. I shouldn't know more about my care than my physician."); issues related to the TG case manager ("I don't know whether [the case manager] is experienced in this position ... she dropped the ball ... she's not proactive."); navigating KPMAS for TG care ("It's already overwhelming navigating the system, but [to have to] be the bridge for two [KP departments] ... is a little frustrating."); and lack of information for TG patients ("Very few people actually know what's covered").

Conclusion: Improvements in the quality of care for TG patients requires targeted efforts on patient-focused initiatives. Resources to further grow the KPMAS TG health services program (such as hiring personnel, training staff/ providers, website support, and a dedicated TG advocate) could result in considerable achievements in TG quality of care at KPMAS.

Keywords: quality of care, qualitative research, quality improvement, patient experience/satisfaction

\section{P16.06}

Developing Patient-Informed Health Education Materials in an Integrated Health System: The Case of Safe Fish Consumption

Jennifer Renner, ${ }^{1}$ Abigail S. Katz, ${ }^{2}$ Thomas Kottke, ${ }^{2}$ Patricia McCannn, ${ }^{3}$ Lisa Harvey, ${ }^{1}$ Jeanne Mettner, ${ }^{1}$ Ruth Taswell, ${ }^{1}$ Jeanette Y. Ziegenfuss ${ }^{1}$

${ }^{1}$ HealthPartners Institute, Bloomington, $\quad M N$; ${ }^{2}$ HealthPartners, Bloomington, MN; ${ }^{3}$ Minnesota Department of Health, Saint Paul, MN

Background: It is important that health materials for patients be designed with their input, especially for a topic as nuanced as safe fish consumption (the message: eat fish, but be informed and intentional about which species you choose to eat). As a large integrated health system, we have numerous existing avenues - our research and education institute (Institute), care delivery system, and health plan to collect this type of input.

Methods: In partnership with the Minnesota Department of Health (MDH), we developed materials promoting safe fish consumption for women who are or could become pregnant. Initial research, not detailed here, involved focus groups of women in the target population to inform alpha versions of our materials. To further refine these materials, we tapped into our organization's feedback avenues, reaching out to HealthPartners' myVoice marketing panel (1200 plan members and/or patients willing to share insights about specific topics via online panel 1-2 times per month) and Patient Council (15 plan members who meet in person 
10 times per year). Findings from these member/patient feedback sources were used to refine the alpha materials with expertise from the Institute's patient education department. Results: The myVoice panel reported trust in both HealthPartners and the Institute but more familiarity with the HealthPartners brand, which informed the logo used on our materials. Patient Council confirmed an unfamiliarity with the Institute and reported confusion about the title of our brochure, which resulted in a new name, Choose Your Fish, to clarify its contents.

Conclusion: The ability to make use of the numerous established feedback pathways built into our integrated system has been invaluable, and the partnership was successful. Input from patients and members through embedded feedback sources has resulted in fish consumption materials designed for and informed by patients. With proper formal partnerships, it is possible to simultaneously meet $\mathrm{MDH}$ and HealthPartners goals using the extant resources of our organization.

Keywords: health promotion, prevention, screening, engagement of stakeholders, health care organization, qualitative research

\section{P16.07}

The Patient Engagement in Research Scientific Interest Group: Connect With and Learn From Stakeholders to Enhance Patient-Engaged Research

Leslie Wright, ${ }^{1}$ Ellis Dillon, ${ }^{2}$ Sarah Madrid, ${ }^{1}$ Gina Napolitano, ${ }^{3}$ Leah Tuzzio, ${ }^{4}$ Mary Schramke ${ }^{5}$

${ }^{1}$ Institute for Health Research, Kaiser Permanente Colorado, Denver, CO; ${ }^{2}$ Palo Alto Medical Foundation Research Institute, Sutter Health, Mountain View, CA; ${ }^{3}$ Patient Advisory Board, Kaiser Permanente Colorado, Denver, CO; ${ }^{4}$ Kaiser Permanente Washington Health Research Institute, Seattle, WA; ${ }^{5}$ Patient and Family Advisory Councils, Sutter Health, Mountain View, CA

Background: The Health Care Systems Research Network (HCSRN) Patient Engagement in Research Scientific Interest Group (PER SIG) aims to help researchers build infrastructure for PER and to develop generalizable tools to enhance engagement efforts. We are a network of researchers committed to and experienced with patient engagement who want to collaborate and build support and best practices for PER.

Methods: The PER SIG began in 2013 as the Member Engagement in Research Work Group, following the inception of a member engagement toolkit developed by research staff at Kaiser Permanente Colorado. Attendees motivated to further PER within HCSRN conducted a baseline assessment of organizational readiness to conduct PER and created the Patient Engagement Workbook, a set of standards for guiding engagement practices. The workbook was presented at the 2014 meeting and generated interest in patient-engaged research. This group became a formal SIG in spring 2014 and includes researchers and patient partners from within and outside of HCSRN who contribute to discussions at monthly webinars, offer consulting services, and co-lead PER SIG workshops. PER SIG webinars are well attended and cover novel methods for expanding patient engagement.

Results: The PER SIG currently has 122 members, with 50\% being very active. We've hosted numerous presentations and consultations during our monthly webinars and presented at the 2015-2017 HCSRN annual meetings. During the 2016 conference we hosted a half-day workshop on patientengagement fundamentals, sustaining engagement, and gaps in assessing impact. This was followed by a 1.5-day workshop focused entirely on developing methods for assessing impact, funded by a Patient-Centered Outcomes Research Institute (PCORI) Eugene Washington award. These workshops have generated 2 publications and many collaborations within HCSRN and with other organizations. Patient partners have expressed that our collaboration across research institutions communicates sincerity.

Conclusion: The PER SIG is a rich resource for fostering patient engagement within HCSRN and is recognized by PCORI and our local organizations as a leader in PER. It has created a virtual community of practice that shares tools, manuscripts, and questions through its listserv and will ensure continued research support for PER despite fluctuations in national funding for this work.

Keywords: research ethics, patient-provider communication, patient experience/satisfaction, engagement of stakeholders, patient-reported outcomes/function, clinical decisionmaking, dissemination, implementation, innovation

\section{P16.08 \\ Assessing Patient Recruitment Strategies by Race in the Multicenter Randomized HYpertension and VALUEs (HYVALUE) Trial}

Christine Truong, ${ }^{1}$ Stacie L. Daugherty, ${ }^{2}$ Haihong Hu, ${ }^{1}$ Josh Durfee, ${ }^{3}$ Kris Wain, ${ }^{4}$ Julie Maertens, ${ }^{5}$ Jennifer McCance, ${ }^{4}$ Hilde Heyn, ${ }^{3}$ Amanda Skenadore,${ }^{5}$ Leslie Wright, ${ }^{4}$ Suzanne Dircksen, ${ }^{3}$ Rebecca Hanratty, ${ }^{3}$ John Steiner, ${ }^{4}$ Irene Blair, ${ }^{6}$ L. Miriam Dickinson, ${ }^{5}$ Laura Helmkamp, ${ }^{5}$ Edward P. Havernek, ${ }^{3}$ Suma Vupputuri ${ }^{1}$

${ }^{1}$ Mid-Atlantic Permanente Research Institute, Kaiser Permanente Mid-Atlantic States, Rockville, MD; ${ }^{2}$ University of Colorado School of Medicine, Aurora, CO; ${ }^{3}$ Denver Health and Hospital Authority, Denver, CO; ${ }^{4}$ Kaiser Permanente Colorado, Denver, CO; ${ }^{5}$ Adult and Child Consortium for Health Outcomes Research and Delivery Science, Aurora, $\mathrm{CO} ;{ }^{6}$ University of Colorado Boulder, Boulder, $\mathrm{CO}$

Background: Patient recruitment studies use a variety of methods; however, little is known about recruitment strategies on response rates by race. The purpose of this analysis is to describe patient response, by race, to different 
recruitment strategies employed at 3 study sites for a 6-month pragmatic clinical trial assessing the effectiveness of values affirmation on reducing racial disparities in antihypertensive medication adherence.

Methods: HYVALUE is an ongoing patient-level randomized controlled trial being conducted at Denver Health (DH), Kaiser Permanente Colorado (KPCO) and Kaiser Permanente Mid-Atlantic States (KPMAS). White and black adult patients with uncontrolled blood pressure, who have an upcoming appointment with their primary care provider, are eligible for inclusion. All sites identify potentially eligible patients from the electronic medical record and perform recruitment telephone calls. The 3 sites customize additional recruitment methods including use of introductory mailed letters (KPMAS), voicemail messages left for all eligible patients (KPCO), and dual enrollment of patients engaged in a similar study (DH). We compared the recruitment rate (number of patients completing screening form / number of patients called) by race for each site.

Results: During the first 6 months of HYVALUE, 206 patients were recruited out of 714 (28.9\%) called. The proportion of white and black patients at each site varies. The recruitment rates by site were $32 \%$ at $\mathrm{DH}, 32 \%$ at $\mathrm{KPCO}$, and $26 \%$ at KPMAS. At $\mathrm{DH}$, the recruitment rate by race was $27.8 \%$ white and $34.4 \%$ black. At KPCO, the recruitment rate was $33.9 \%$ white and $27.9 \%$ black. At KPMAS, the recruitment rate was $18.8 \%$ white and $26.7 \%$ black. Further, KPMAS had an $8.5 \%$ patient response to the mailings alone (ie, patients contacted the study by phone or mail), with the majority of these responses from black patients. Of the 206 patients that were recruited, 154 patients were enrolled (74.8\%).

Conclusion: The initial recruitment experience of HYVALUE demonstrates little difference in recruitment by race at $\mathrm{DH}$ and $\mathrm{KPCO}$, but higher recruitment rates of blacks compared to whites at KPMAS. These results emphasize the need for multimodal recruitment strategies based on the underlying sampling population to optimize the recruitment rates in multicenter trials.

Keywords: cardiovascular disease, human subjects research, racial/ethnic differences, health care, clinical trials

\section{P16.09}

\section{Cost-Effectiveness of Physician Incentives for Resident Feedback}

Jessie Nelson, ${ }^{1}$ Hilary Simon, ${ }^{2}$ Callie Schnitker, ${ }^{2}$ Sandi Wewerka ${ }^{3}$

${ }^{1}$ Emergency Medicine, Regions Hospital, Saint Paul, MN; ${ }^{2}$ Regions Hospital Emergency Medicine Residency, Saint Paul, MN; ${ }^{3}$ Critical Care Research Center, Regions Hospital, Saint Paul, MN

Background: Emergency medicine (EM) residents in the United States must attain predetermined educational milestones set forth by the Accreditation Council for
Graduate Medical Education for graduation. Facultygenerated feedback has been shown to improve residents' progression toward these ends. Unfortunately, feedback quality has often been lacking in residency education. Our EM residency introduced cards completed by faculty at end-of-shift to highlight and hopefully improve direct feedback given to residents, but initial use by faculty was inconsistent. In response to resident-perceived deficiencies in end-of shift feedback, small annual financial incentives were offered for improved percentage of end-of-shift cards completed (as a proxy for feedback quantity) as well as resident-rated quality of each faculty physician's end-ofshift feedback.

Methods: The aim of this study was to determine if faculty-directed financial incentives improved shift card completion percentage and resident-perceived quality of faculty-to-resident feedback. In this institutional review board-approved retrospective observational study, feedback was compared pre- and postimplementation of a financial bonus policy. Eligible faculty were employed throughout a 1-year time period before and after implementation of the incentive $(n=24)$. Quantity of feedback was calculated as the number of cards completed per shift worked with at least one resident. Quality of feedback was determined from annual surveys asking residents to rate the quality of feedback received from each faculty member over the previous year on a scale from 1 to 9 . The average faculty score before and after incentive implementation was determined and compared.

Results: Mean percentage of shift cards completed was significantly higher postincentive (mean: 129.51\%, SD: 42.82) compared to preincentive (mean: 26.54\%, SD: 41.69); $\mathrm{P}<0.01$. Mean resident-perceived feedback quality scores were similar preincentive (mean: 7.52, SD: 0.63) and postincentive (mean: 7.71, SD: 0.57); $\mathrm{P}=0.66$.

Conclusion: This financial incentive is associated with significantly increased quantity of end-of-shift card completion, but no difference in resident-perceived quality of end-of-shift feedback.

Keywords: human subjects research, health care workforce, hospital

P16.10

Assessing Researcher Experience and Attitudes Toward Patient Engagement in Research

\section{Michael Gionfriddo}

${ }^{1}$ Geisinger Health, Forty Fort, PA

Background: Patient engagement is becoming increasingly recognized as not only an ethical approach to research but also an important way to make research more applicable and relevant to the ultimate end-user: the patient. The PatientCentered Outcomes Research Institute has mandated that all proposals have an engagement plan defining how 
the proposed research will engage relevant stakeholders, including patients, throughout the research, from topic selection through dissemination activities. We conducted a survey to better understand researchers' experience with and attitudes toward patient engagement in research at our institution (Geisinger Health).

Methods: The survey was sent to all staff listed as primary investigator, co-primary investigator, or co-investigator on a research protocol approved by Geisinger's institutional review board.

Results: We had a $24 \%$ response rate (140 of 588). Of those who responded, $81 \%$ conducted human subjects research. While the majority responded that they involved patients as participants in research, few engaged patients in other ways, with the second most common way (18\%) being having patients help them identify topics and questions for research. Researchers reported barriers to involving patients in research, with the most common being that it was logistically challenging (48\%) and that it takes too much time (34\%). Of those who reported involving patients in their research in some way, 53\% reported substantial benefits. Of all respondents, $46 \%$ were interested in learning more about involving patients in research, with most wanting to learn about resources for supporting and facilitating patient involvement in research and effective strategies for initiating and sustaining patient involvement in research.

Conclusion: These data will be used to help us refine our organization's strategies for promoting and facilitating patient engagement in research.

Keywords: engagement of stakeholders

P16.11

Preliminary Impact of a Novel Team-Based Competition on Faculty Attendance to Weekly Residency Didactics

\author{
Jessie Nelson, ${ }^{1}$ Alan Sazama, ${ }^{2}$ Kristi Grall, ${ }^{1}$ Sandi \\ Wewerka ${ }^{3}$
}

${ }^{1}$ Emergency Medicine, Regions Hospital, Saint Paul, MN; ${ }^{2}$ Regions Hospital Emergency Medicine Residency, Saint Paul, MN; ${ }^{3}$ Critical Care Research Center, Regions Hospital, Saint Paul, $M N$

Background: Our residency implemented a novel teambased competition called the Regions Conference Cup with several goals for the academic year, including improved faculty didactic conference attendance, improved ontime attendance, and improved conference evaluation completion rates. Team captains were chosen from residency leadership. Opportunities for scoring were determined by residency leadership, a priori, based on preexisting goals. Residents and faculty were then randomly assigned to teams. Players (residents and faculty) score points for their team by attending conference, attending conference on time, and completing evaluations. The inaugural season of the competition was from August 2016 to May 2017. This planned preliminary analysis details the impact of the first 3 months of "competition" on emergency medicine faculty attendance, resident tardiness, and conference evaluation completion.

Methods: We retrospectively reviewed faculty attendance data from August to October in 2014, 2015, and 2016. Percentage of faculty attendance was calculated by noting the number of faculty attending at least 1 hour of conference and dividing it by the total number of clinically active faculty. Number of tardies to conference was compared within the time period of August 2016October 2016. Finally, we reviewed conference evaluation completion rates from August to October in 2014 and 2015 and compared them to 2016 completion rates.

Results: Faculty conference attendance in AugustOctober of 2014 and 2015 (pre-Conference Cup) averaged 8.17 (SD: 2.88, 95\% CI: 6.33-10.00) and 10.25 (SD: 3.44, 95\% CI: 8.06-12.44), respectively. In August-October 2016, faculty conference attendance was 13.25 (SD: 3.82 , 95\% CI: 10.82-15.67). Average number of residents tardy to conference trended downward in each month of the Conference Cup (August: 7, September: 5.3, October: 4.6). Conference evaluations completed for August-October was 9.09 (95\% CI: 2.48-15.70) in 2014; 10.73 in 2015 (95\% CI: 4.96-16.50), and 23.3 in 2016 (95\% CI: $17.90-$ 28.70).

Conclusion: The first months of Conference Cup competition were associated with increased percentage of emergency medicine faculty attendance at weekly residency didactic conference compared to 2014 and trending to significant improvement over 2015. Conference evaluation completion rates similarly show a strong trend toward a significant increase. Within the time period of the Conference Cup, faculty and resident tardy arrivals are trending downward.

Keywords: health care organization, workforce, hospital, quality improvement

P16.12

Colorado Patient Partners in Research: Engaging at Multiple Intersections in Patient Health

Leslie Wright, ${ }^{1}$ Tammy Bellofatto, ${ }^{1}$ Selena Dunham, ${ }^{1}$ Charlene Barrientos-Ortiz ${ }^{1}$

\section{${ }^{1}$ Colorado Patient Partners in Research, Denver, CO}

Background: Colorado Patient Partners in Research (CoPPiR) is a Denver Health/Kaiser Permanente partnership designed to raise patients' voice, involvement, and leadership in Colorado health research. Our work centers on three approaches: a patient stakeholder council (CoPPiR 
Council); the CoPPiR Network connecting researchers with patient partners; and social media promoting patientcentered research approaches and directing people to the CoPPiR Network. The CoPPiR Council governs all three aspects of our work.

Methods: The CoPPiR Council provides broad guidance to Colorado-based research shops and individual researchers on patient-centered approaches to research design, implementation, data interpretation, and dissemination. To ensure a safe and respectful place for all council members to be heard, and for engagement to be beneficial, various trust-building strategies have been implemented: a member recruitment plan co-developed by community advisors and community research liaisons; opening each meeting with a Conocimiento question; and capacity-building trainings emphasizing co-learning and exploring the intersection of patient engagement and research partnership.

Results: In the span of 18 months the council has grown to 9 very diverse, core members. They have completed 6 trainings specifically tailored to council goals, developed the CoPPiR Council and Network governance document, presented at 3 statewide conferences, attended 2 PatientCentered Outcomes Research Institute annual meetings, and provided research consultation.

Conclusion: The CoPPiR Council and CoPPiR Network form a model for patient-engaged research that can be replicated throughout the health enterprise

Keywords: research ethics, patient-provider communication, engagement of stakeholders, health care organization, clinical decision-making, patient-reported outcomes/function, dissemination, implementation, innovation, access to services, patient experience/ satisfaction

\section{P16.13}

\section{Critical Care Research Center: A Research Platform for the Community Hospital}

\section{David Dries, ${ }^{1}$ Sandi Wewerka, ${ }^{1}$ Paula Miller ${ }^{1}$ \\ ${ }^{1}$ Critical Care Research Center, Regions Hospital, Saint Paul, $M N$}

Background: Regions Hospital, due in large part to specialty care service lines including Level I trauma and burn management along with critical care, is well positioned to advance clinical practice through research. However, the hospital lacks the academic infrastructure of a university medical center.

Methods: In partnership with the HealthPartners Institute, a group of physicians in areas including emergency medicine, critical care, burn care, trauma, emergency medical services, and hospital medicine created a research center funded with grants from committed departments. The Critical Care Research Center (CCRC) hired a class of research interns, typically students in transition between undergraduate and graduate programs, trained these individuals in research practice, and contracted for statistical and study design support from the Institute. CCRC also provides research experience to trainees at the University of Minnesota who do not otherwise have access to this patient population. Local leadership comes from a hospital-based physician advisory group.

Results: Over the last 5 years 176 projects have been initiated, resulting in 115 research presentations and generating $\$ 2.75$ million of external funding, complemented by $\$ 570,000$ from internal grants. Internal funding has allowed enhanced support for study design, data analysis, and manuscript preparation, resulting in an increased transition to external funding sources. CCRC also has attracted funding from the Anderson Foundation to further develop its infrastructure. In 2017, Regions Hospital leadership sponsored a community survey to evaluate community attitude and awareness of ongoing hospital programs in our region. One point of emphasis in the survey was respondent awareness of research and clinical trials. When Regions Hospital was considered, two-thirds of Twin Cities consumers had a more positive impression of the hospital associated with the conduct of research and clinical trials.

Conclusion: Driven by a multidisciplinary physician commitment and organization support, this research center model allows participation in multiple study types without the need for extensive academic infrastructure.

Keywords: human subjects research, health care organization, engagement of stakeholders, research administration, contracting, operations, hospital

\section{POPULATION, COMMUNITY, AND WORKSITE HEALTH}

\section{P17.01 \\ Managing Population Health: Expanding Hemoglobin A1c Testing -- The First Step in Controlling Diabetes and Prediabetes}

Glenn Melnick, ${ }^{1}$ June O'Leary, ${ }^{1}$ Ning Fu, ${ }^{1}$ Janelle Howe, ${ }^{2}$ Jeremy Rich ${ }^{3}$

${ }^{1}$ University of Southern California, Los Angeles, CA; ${ }^{2}$ DaVita HealthCare Partners, El Segundo, CA; ${ }^{3}$ HealthCare Partners Institute for Applied Research and Education, El Segundo, CA

Background: It is widely recognized that one of the keys to successfully managing population health is improved measurement of health status. Recognizing this issue, DaVita HealthCare Partners implemented a program to expand the number of Southern California members 
who receive a hemoglobin A1c test as a tool to diagnosis prediabetes/diabetes and to improve glycemic control in current diabetic patients. It was recently estimated that nearly half of adult Californians (13 million) have prediabetes or undiagnosed diabetes.

Methods: Laboratory results were searched for hemoglobin A1c tests results (Logical Observation Identifiers Names and Codes, or LOINC ${ }^{\circledR}$ codes, 4548-4, 4549-2, 59261-8, 62388-4, and 17856-6) during the period January 1, 2007, through December 31, 2016. In the greater Los Angeles area, DaVita HealthCare Partners serves over half a million members.

Results: Of the more than 2.5 million hemoglobin A1c test results found during the study period, nearly all (over 99\%) were due to LOINC code 4548-4, which is the standard in the United States today. In 2009, an international expert committee recommended the hemoglobin A1c test be available (in addition to fasting plasma and oral glucose tolerance tests) to help diagnose type 2 diabetes and prediabetes. The total number of hemoglobin A1c tests administered increased substantially over the 10-year study period, from 81,864 in 2007 to a high of 509,067 tests in 2015 and 414,726 tests in 2016. The number of new members tested in each year averaged 54,756 over the 10 -year period. The number of tests per member averaged 1.81 per year with slight year-to-year variation.

Conclusion: DaVita HealthCare Partners successfully expanded the coverage of hemoglobin A1c testing among their Southern California population. The increase is largely due to expanding the number of members tested rather than an increase in the number of tests per member. A multifaceted approach, involving both providers and patients, appears effective at increasing hemoglobin A1c testing among those previously not tested. The results of ongoing interviews that describe the intervention to increase testing, including key components, barriers, and lessons learned, will be incorporated as part of this research.

Keywords: observational study, patient-provider communication, engagement of stakeholders, diabetes, qualitative research, chronic disease, clinical practice patterns/guidelines

\section{P17.02}

\section{Evaluating and Improving Physician Well- Being: Insights From Frontline Physicians and Organizational Leaders at a Large Multispecialty Group Practice}

\author{
Yan Yang, ${ }^{1}$ Ellis Dillon, ${ }^{1}$ Amy Meehan, ${ }^{1}$ Jinnan Li, ${ }^{1}$ \\ Dominick Frosch, ${ }^{1}$ Robert Nordgren, ${ }^{1}$ Ming Tai- \\ Seale ${ }^{1,2}$
}

${ }^{1}$ Palo Alto Medical Foundation, Sutter Health, Palo Alto, CA; ${ }^{2}$ University of California, San Diego, San Diego, CA
Background: About half of physicians report burnout nationally. Physician surveys revealed that $36 \%$ of all physicians and $44 \%$ of primary care physicians (a specialty already facing a workforce shortage) in this large multispecialty health system reported symptoms of burnout. It is an organizational priority to address physician burnout by exploring factors contributing to burnout and potential interventions to enhance well-being.

Methods: The study included a survey, the results of which helped guide in-depth interviews. The survey, which regarded burnout (using a nonproprietary single-item measure) and factors affecting burnout, was delivered to all providers at 5 multispecialty medical groups. Univariate and bivariate analyses were conducted to study providers' self-care practices, perceptions of work environment, and their associations with well-being. The in-depth interviews were conducted with frontline physicians and organizational leaders and included discussion of factors identified as associated with well-being/burnout via the surveys and what changes might be made to improve well-being. Two coders analyzed interviews using deductive and inductive coding methods to identify themes and conceptual domains related to well-being.

Results: Survey response rate was 47\%; $40 \%$ reported burnout symptoms (41\% among physicians and 33\% among nonphysician health care providers). Average life satisfaction was 80 (SD: 23) on a scale of $0-100 ; 82 \%$ perceived having control over work schedule; $9 \%$ felt that physicians are highly valued; $67 \%$ reported calm atmosphere in work area. Lower burnout rates were found for providers who perceived having good control over their work schedule ( $32 \%$ vs $75 \% ; \mathrm{P}<0.001$ ), who felt physicians were highly valued by the organization ( $16 \%$ vs $43 \% ; \mathrm{P}=0.009)$, who reported a calm atmosphere in their primary work area (32\% vs $57 \% ; \mathrm{P}<0.001)$, who slept $\geq 7$ hours/night $(27 \%$ vs $45 \% ; \mathrm{P}=0.003)$, and who exercised $\geq 2$ days/week $(35 \%$ vs $50 \%$; $\mathrm{P}=0.017$ ). Interviews were conducted with 17 key informants. Interviewees prioritized organizational factors over individual factors. Reducing physician workload and electronic health record (Epic) in-basket work (described as "a virtual hamster wheel"), increasing feeling valued by the organization, and creating meaning in work were identified as most salient for addressing well-being. A factor identified as protective of well-being is a sense of community at work. Suggestions included maximizing resources so that physicians are working at the top their skill level, having the right support staff available, and rethinking full-time equivalence (FTE) definitions. Interviewees suggested that addressing these topics would have a trickle-down effect on workload, work-life balance, and autonomy.

Conclusion: Burnout symptoms varied by self-care practices and perceived work experience. Addressing in-basket burden, allocating sufficient support staff, and maximizing resources may have a ripple effect and improve other dimensions related to improving physician wellbeing. Changes at every level of the organization must be coordinated to maximize physician well-being. 
Keywords: health care workforce, observational study, survey research and methods

\section{P17.03}

\section{Hotspots of Triple Aim Opportunity: Identifying Geographic Clusters of Potentially Avoidable Emergency Department Visits and Costs}

\section{Bjorn Westgard, ${ }^{1}$ David Warren ${ }^{1}$}

\section{${ }^{1}$ HealthPartners, Minneapolis, $M N$}

Background: Poor access to care leads many patients in the United States to use the emergency department (ED) for conditions that may be "avoided" by timely ambulatory care. The NYU/Billings ED algorithm is one of several systems for estimating these potentially-avoidable ED visits. Health care systems use these tools to improve patient outcomes and lower costs through actuarial risk stratification and individual interventions. We used geographic information systems (GIS) and spatial analysis to examine if geographic clusters exist and overlap for potentially avoidable visits and high institutional costs of care, suggesting potential opportunities for Triple Aim interventions.

Methods: 74,644 ED visits to Regions Hospital were analyzed using the NYU/Billings ED algorithm as applied to up to 5 discharge diagnoses to determine the probability that each condition needed ED care, selecting the condition with the highest probability of being emergent. Trauma, mental health, and drug/toxicology cases were excluded. Internal cost-accounting figures for each ED visit were then obtained, including associated inpatient costs when applicable. For each ED visit, the potential visit avoidability, the costs for the visit, and other visit characteristics (eg, acuity, disposition) were then aggregated by block group for cluster and hotspot analyses using ArcGIS.

Results: Spatial statistical analysis of average acuity, avoidability, and cost per visit did not show significant differences among neighborhoods. However, GIS analysis demonstrates significant clustering and overlap of both potentially avoidable ED visits and high total and marginal costs in a small but significant number of block groups. These neighborhoods have similar 2010 U.S. Census characteristics as well as similar propensities for ED use based on data from the Minnesota Department of Health. Notably, they also have significantly fewer primary care resources available.

Conclusion: Geospatial analysis of ED visits for potential avoidability and cost can identify opportune neighborhoods for educational, primary care, or alternative health care interventions to improve care and lower costs through improvements in public health. Our future analyses and modeling will integrate patient-level factors in order to specify which conditions, patients, and neighborhoods are most likely to benefit from Triple Aim efforts to reduce potentially avoidable ED visits and their costs by improving health care access.

Keywords: geographic information systems, spatial factors, ambulatory/outpatient care, access to services

P17.04

Assessing the Cost Sustainability of a Behavioral Economic Intervention to Improve the Availability of Healthy Food to Food Shelf Customers: A Feasibility Analysis

Julia Johnson, ${ }^{1}$ Marna Canterbury, ${ }^{1}$ Thomas Kottke, ${ }^{1}$ Caitlin Caspi, ${ }^{2}$ Katherine Grannon, ${ }^{2}$ Liz Riley ${ }^{3}$

${ }^{1}$ HealthPartners, Stillwater, MN; ${ }^{2}$ Department of Family Medicine and Community Health, University of Minnesota, Minneapolis, MN; ${ }^{3}$ Valley Outreach, Stillwater, MN

Background: The SuperShelf model of food shelf design uses behavioral economic strategies to influence customers' healthy food selection by making the healthy choice the easy choice. But the critical question is whether it is costsustainable for food shelves to implement the model. We are examining the feasibility of defining the relationship between the amount of money the food shelf spends on food, the quantity of food provided to customers, and the nutritional quality of the food. If measurement is feasible, we will test the cost-sustainability of the SuperShelf model. Methods: We implemented the SuperShelf model in two Minneapolis/Saint Paul metro area food shelves. We analyzed food shelf invoices for January to July from the two main food banks before (2016) and after (2017) we implemented SuperShelf to control for seasonality effects on cost and availability. We calculated cost per person per month ("pppm") and pounds of food pppm. Food shelf invoices were scored for nutritional quality using the Food Assortment Sorting Tool. We also analyzed food shelf invoices for two control sites in the same way.

Results: Food shelf assessments showed that SuperShelf was delivered with greater fidelity at intervention site A compared to site B. Neither SuperShelf food shelf increased pppm cost and both were able to provide customers with more food pppm than before the intervention. Additionally, intervention food shelves maintained food quality postintervention. We observed a similar trend in control site A, but an opposite trend in control site B.

Conclusion: We conclude that it is feasible to measure the cost-effectiveness of the SuperShelf model. Preliminary results indicate that food shelves that implement the SuperShelf model can be cost-sustainable and can increase the quantity and amount of healthier foods provided to customers.

Keywords: financial analysis 